\title{
TWISTS OF HILBERT MODULAR FORMS
}

\author{
Thomas R. Shemanske and Lynne H. Walling
}

\begin{abstract}
The theory of newforms for Hilbert modular forms is summarized including a statement of a strong multiplicity-one theorem and a characterization of newforms as eigenfunctions for a certain involution whose Dirichlet series has a prescribed Euler product. The general question of twisting Hilbert modular newforms by arbitrary Hecke characters is considered and the exact level of a character twist of a Hilbert modular form is determined. Conditions under which the twist of a newform is a newform are given. Applications include a strengthening in the elliptic modular case of a theorem of Atkin and Li's regarding the characterization of imprimitive newforms as well as its generalization to the Hilbert modular case, and a decomposition theorem for certain spaces of newforms as the direct sum of twists of spaces of newforms of lower level.
\end{abstract}

\section{INTRODUCTION}

For the case of elliptic modular forms, Hijikata, Pizer and Shemanske [3] show how to decompose a space of newforms as a direct sum of character twists of other spaces of newforms. In particular, these decomposition theorems yield information about how a given newform behaves under character twists: what the exact level of a character twist is, and under what circumstances the twist of a newform is a newform. Atkin and Li [1] consider these specific questions for elliptic modular forms by different methods and with a different perspective. In this paper, we adapt the methods of [1] [5] to investigate similar questions in the case of Hilbert modular forms but with an eye towards the decomposition theorems of [3].

We begin with a summary of the newform theory for Hilbert Modular Forms; in particular, we present both a regular and strong multiplicity-one theorem (Theorems 3.5 and 3.6). While a multiplicity-one theorem follows (at least in principle) from the work of Miyake [7], we give a characterization of newforms as cusp forms which are eigenfunctions for a certain involution and whose associated Dirichlet series has a prescribed Euler product (Theorem 3.7). We then use this characterization to prove that the twist of a Hilbert modular newform by a Hecke character whose conductor is prime to the level is a newform (Theorem 5.5).

To examine twists of newforms by arbitrary Hecke characters, we begin by generalizing Atkin and Li's operator $W_{\mathcal{Q}}$. The definition and properties of this generalized operator are rather delicate, reflecting differences among the various Hecke characters which "extend" the numerical character of the space of cusp forms on which $W_{\mathcal{Q}}$ acts. Using a result of Shimura regarding the special values of Dirichlet series attached to Hilbert modular forms [11] [12], we characterize certain properties of

1991 Mathematics Subject Classification. 11F41.

Key words and phrases. Hilbert modular form, newform, character twists.

Typeset by $\mathcal{A} \mathcal{M} \mathcal{S}-\mathrm{T}_{\mathrm{E}} \mathrm{X}$ 
the pseudo-eigenvalues of $W_{\mathcal{Q}}$ (Theorem 4.2). This eventually allows us to determine conditions under which twisting a newform by a character whose conductor divides the level of the form yields a newform (Theorem 7.1). This theorem extends Theorem 3.1 of [1] which only gives the exact level of a twist, and applies both to Hilbert modular as well as elliptic modular forms. In turn, this theorem allows us to decompose a certain type of space of Hilbert modular newforms as the direct sum of twists of spaces of newforms of lower level (Theorem 7.2), analogous to Theorem 3.14 of [3].

Most of the results of [5] and [1] generalize to the Hilbert modular case. There is an important result concerning the non-vanishing of Hecke eigenvalues (Theorem 3.3 (2)) which we were able to generalize in a significant number of, but not all, cases however, we have been informed that it follows from the representation theory that the result holds in all cases. This result is critical to the determination of when the twist of a newform is again a newform (Theorem 5.8), and is consequently of concern to us here.

\section{Notation}

For the most part we follow the notation of [11] and [12]. However, to make this paper somewhat self-contained, we shall briefly review the basic definitions of the types of functions and operators to be studied here; more details can be found in Shimura's two papers referenced above.

Let $K$ be a totally real number field of degree $n$ over $\mathbb{Q}, \mathcal{O}$ its ring of integers, and $\mathcal{O}^{\times}$and $\mathcal{O}_{+}^{\times}$the groups of units and of totally positive units respectively. Let $\mathfrak{d}$ be the different of $K$. Let $G L_{2}^{+}(K)$ denote the group of invertible matrices with totally positive determinant and $\mathcal{H}$ the complex upper half-plane. Then $G L_{2}^{+}(K)$ acts by fractional linear transformation on $\mathcal{H}^{n}$ via

$$
(A, \tau) \mapsto A \tau=\left(\ldots, \frac{a^{(\nu)} \tau_{\nu}+b^{(\nu)}}{c^{(\nu)} \tau_{\nu}+d^{(\nu)}}, \ldots\right)
$$

where $a^{(\nu)}$ denotes the $\nu^{\text {th }}$ conjugate of $a$ over $\mathbb{Q}$. For $N \in \mathbb{Z}_{+}$, let

$$
\Gamma_{N}=\left\{A \in S L_{2}(\mathcal{O}) \mid A-1_{2} \in N \operatorname{Mat}_{2}(\mathcal{O})\right\} .
$$

For $k=\left(k_{1}, \ldots, k_{n}\right) \in\left(\mathbb{Z}_{+}\right)^{n}$ and $c, d \in K$, let $(c \tau+d)^{k}=\prod_{\nu=1}^{n}\left(c^{(\nu)} \tau_{\nu}+d^{(\nu)}\right)^{k_{\nu}}$. Define $M_{k}\left(\Gamma_{N}\right)$ to be the complex vector space of functions $f$ holomorphic on $\mathcal{H}^{n}$ and at the cusps of $\Gamma_{N}$ such that $f(A \tau)=(\operatorname{det} A)^{k / 2}(c \tau+d)^{k} f(\tau)$ for all $A \in \Gamma_{N}$. Let $M_{k}=\cup_{N=1}^{\infty} M_{k}\left(\Gamma_{N}\right)$.

For $\mathcal{I}$ a fractional ideal and $\mathcal{N}$ an integral ideal, put

$$
\Gamma_{0}(\mathcal{N}, \mathcal{I})=\left\{A \in\left(\begin{array}{cc}
\mathcal{O} & \mathcal{I}^{-1} \mathfrak{d}^{-1} \\
\mathcal{N} \mathcal{I} \mathfrak{d} & \mathcal{O}
\end{array}\right) \mid \operatorname{det} A \in \mathcal{O}_{+}^{\times}\right\} .
$$

By a numerical character $\psi$ modulo $\mathcal{N}$ we mean a character $\psi:(\mathcal{O} / \mathcal{N})^{\times} \rightarrow \mathbb{C}^{\times}$, and by a Hecke character we mean a character $\Psi: K_{A}^{\times} \rightarrow \mathbb{C}^{\times}$which is trivial on $K^{\times}$. (In general, we use lower case Greek letters to denote numerical characters and upper case Greek letters to denote Hecke characters.) As in Shimura [11] [12], for $\psi$ a numerical character $\bmod \mathcal{N}$ and $\theta$ a character of $\mathcal{O}_{+}^{\times}$of finite order, define $M_{k}\left(\Gamma_{0}(\mathcal{N}, \mathcal{I}), \psi, \theta\right)$ to be the set of all $f \in M_{k}$ such that

$$
f(A \tau)=(\operatorname{det} A)^{-k / 2} \psi(a) \theta(\operatorname{det} A)(c \tau+d)^{k} f(\tau) .
$$


We always assume that $\psi(\varepsilon) \theta\left(\varepsilon^{2}\right)=\operatorname{sgn}(\varepsilon)^{k}$ for all $\varepsilon \in \mathcal{O}^{\times}$since $M_{k}\left(\Gamma_{0}(\mathcal{N}, \mathcal{I}), \psi, \theta\right)=$ $\{0\}$ otherwise. Now, there exists an $m \in \mathbb{R}^{n}$ such that $\theta(\varepsilon)=\varepsilon^{i m}$ for all $\varepsilon \in \mathcal{O}^{\times}$; while $m$ is not uniquely determined, it will be fixed throughout this paper.

Let $\mathcal{I}_{1}, \mathcal{I}_{2}, \ldots \mathcal{I}_{h}$ be a set of representatives of the strict ideal classes of $K$, $\Gamma_{\lambda}=\Gamma_{\lambda}(\mathcal{N})=\Gamma_{0}\left(\mathcal{N}, \mathcal{I}_{\lambda}\right)$, and put

$$
\mathfrak{M}_{k}(\mathcal{N}, \psi, \theta)=\prod_{\lambda=1}^{h} M_{k}\left(\Gamma_{\lambda}, \psi, \theta\right) .
$$

We shall study the forms identified with $h$-tuples $\left(f_{1}, f_{2}, \ldots, f_{h}\right) \in \mathfrak{M}_{k}(\mathcal{N}, \psi, \theta)$.

For notational convenience in handling the problems introduced by class number $h>1$, we follow Shimura and describe Hilbert modular forms as functions on an idele group as follows. Let $K_{A}^{\times}$be the idele group of $K$ and $G_{A}$ the adelization of $G L_{2}(K)$. With the usual identifications, we may view $G_{A}=G L_{2}\left(K_{A}\right) ; G_{K}=$ $G L_{2}(K)$ embedded as the diagonal in $G_{A} ; G_{\infty}=G L_{2}(\mathbb{R})^{n}$ the archimedean part of $G_{A}$; and $G_{\infty+}=G L_{2}^{+}(\mathbb{R})^{n}$. For an integral ideal $\mathcal{N}$ of $\mathcal{O}$, let

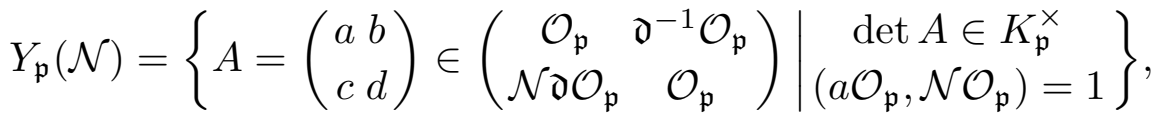

$$
\begin{aligned}
& W_{\mathfrak{p}}(\mathcal{N})=\left\{x \in Y_{\mathfrak{p}}(\mathcal{N}) \mid \operatorname{det}(x) \in \mathcal{O}_{\mathfrak{p}}^{\times}\right\},
\end{aligned}
$$

and put

$$
Y=Y(\mathcal{N})=G_{A} \cap\left(G_{\infty+} \times \prod_{\mathfrak{p}} Y_{\mathfrak{p}}(\mathcal{N})\right), \quad W=W(\mathcal{N})=G_{\infty+} \times \prod_{\mathfrak{p}} W_{\mathfrak{p}}(\mathcal{N}) .
$$

For $\widetilde{a} \in K_{A}^{\times}$and $\mathcal{N}$ an integral ideal, let $\widetilde{a}_{\infty}$ denote the archimedean part of $\widetilde{a}$, $\widetilde{a}_{0}$ the finite part of $\widetilde{a}$, and $\widetilde{a}_{\mathcal{N}}$ the $\mathcal{N}$-part of $\widetilde{a}$. The numerical character $\psi$ : $(\mathcal{O} / \mathcal{N})^{\times} \rightarrow \mathbb{C}^{\times}$induces a character $\psi_{Y}: Y \rightarrow \mathbb{C}^{\times}$by $\psi_{Y}\left(\left(\begin{array}{l}\widetilde{a} * \\ * *\end{array}\right)\right)=\psi\left(\widetilde{a}_{\mathcal{N}} \bmod \mathcal{N}\right)$.

Now, fix a set of ideles $\widetilde{t}_{\lambda} \in K_{A}^{\times},\left(\widetilde{t}_{\lambda}\right)_{\infty}=1$ with $\mathcal{I}_{\lambda}=\widetilde{t}_{\lambda} \mathcal{O}$, and let $x_{\lambda}=$ $\left(\begin{array}{cc}1 & 0 \\ 0 & \tilde{t}_{\lambda}\end{array}\right) \in G_{A}$; also fix an idele $\widetilde{t}_{\mathfrak{d}}$ with $\left(\widetilde{t}_{\mathfrak{d}}\right)_{\infty}=1$ and $\widetilde{t}_{\mathfrak{d}} \mathcal{O}=\mathfrak{d}$. Then by strong approximation, we have

$$
G_{A}=\bigcup_{\lambda=1}^{h} G_{K} x_{\lambda} W=\bigcup_{\lambda=1}^{h} G_{K} x_{\lambda}^{-\iota} W
$$

where $\iota$ denotes the canonical involution on $2 \times 2$ matrices. Finally, given an $h$-tuple of functions $\left(f_{1}, \ldots, f_{h}\right) \in \mathfrak{M}_{k}(\mathcal{N}, \psi, \theta)$, define a function $\mathbf{f}: G_{A} \rightarrow \mathbb{C}$ by

$$
\mathbf{f}\left(\alpha x_{\lambda}^{-\iota} w\right)=\psi_{Y}\left(w^{\iota}\right) \operatorname{det}\left(w_{\infty}\right)^{i m}\left(f_{\lambda} \mid w_{\infty}\right)(\mathbf{i}) \quad \text { for } \alpha \in G_{K} \text {, and } w \in W(\mathcal{N})
$$

where $\mathbf{i}=(i, \ldots, i)($ with $i=\sqrt{-1})$ and where

$$
f_{\lambda} \mid\left(\begin{array}{ll}
a & b \\
c & d
\end{array}\right)(\tau)=(a d-b c)^{k / 2}(c \tau+d)^{-k} f_{\lambda}\left(\frac{a \tau+b}{c \tau+d}\right) .
$$

As in [11] [12], one can identify $\mathfrak{M}_{k}(\mathcal{N}, \psi, \theta)=\prod_{\lambda=1}^{h} \mathcal{M}_{k}\left(\Gamma_{\lambda}, \psi, \theta\right)$ with the set of functions f : $G_{A} \rightarrow \mathbb{C}$ which satisfy: 
(1) $\mathbf{f}(\alpha x w)=\psi_{Y}\left(w^{\iota}\right) \mathbf{f}(x)$ for all $\alpha \in G_{K}, x \in G_{A}, w \in W(\mathcal{N}), w_{\infty}=1$, and

(2) For each $\lambda$ there exists an element $f_{\lambda} \in M_{k}$ such that $\mathbf{f}\left(x_{\lambda}^{-\iota} y\right)=\operatorname{det}(y)^{i m}\left(f_{\lambda} \mid y\right)(\mathbf{i})$ for all $y \in G_{\infty+}$.

Henceforth, the space of such functions will be denoted $\mathfrak{M}_{k}(\mathcal{N}, \psi, m)$, where $m \in \mathbb{R}^{n}$ is fixed and satisfies $\theta(\varepsilon)=\varepsilon^{i m}$ for all $\varepsilon \in \mathcal{O}^{\times}$. We use $\mathfrak{S}_{k}(\mathcal{N}, \psi, m)$ to denote the subspace of cusp forms.

With $m$ as above, define $\psi_{\infty}: K_{A}^{\times} \rightarrow \mathbb{C}^{\times}$by setting $\psi_{\infty}(\widetilde{a})=\operatorname{sgn}\left(\widetilde{a}_{\infty}\right)^{k}\left|\widetilde{a}_{\infty}\right|^{2 i m}$. If for $\widetilde{s} \in K_{A}^{\times}$we define $\mathbf{f}^{\tilde{s}}(x)=\mathbf{f}(\widetilde{s} x)$, then $\mathbf{f} \rightarrow \mathbf{f}^{\tilde{s}}$ induces a unitary representation of $K_{A}^{\times}$in $\mathfrak{M}_{k}(\mathcal{N}, \psi, m)$ which decomposes into a direct sum of irreducible subrepresentations. By Schur's lemma (since $K_{A}^{\times}$is abelian) the irreducible subrepresentations are all 1-dimensional. For a character $\Psi$ of $K_{A}^{\times}$, let $\mathcal{M}_{k}(\mathcal{N}, \Psi)$ denote the subspace of $\mathfrak{M}_{k}(\mathcal{N}, \psi, m)$ consisting of all functions f for which $\mathbf{f}^{\tilde{s}}=\Psi(\widetilde{s}) \mathbf{f}$, and let $\mathcal{S}_{k}(\mathcal{N}, \Psi) \subset \mathcal{M}_{k}(\mathcal{N}, \Psi)$ denote the subspace of cusp forms. Since $\mathbf{f}^{s}=\mathbf{f}$ for $s \in K^{\times}, \mathcal{M}_{k}(\mathcal{N}, \Psi)$ is nontrivial only when $\Psi$ is a Hecke character. Note that from equation $(9.22)$ of $[12]$, we have that $\Psi(\widetilde{a})=\psi\left(\widetilde{a}_{\mathcal{N}}\right) \psi_{\infty}(\widetilde{a})$ for all $\widetilde{a} \in K_{\infty}^{\times} \prod_{\mathfrak{p}} \mathcal{O}_{\mathfrak{p}}^{\times}$. Thus, by a Hecke character extending $\psi \psi_{\infty}$ we shall mean a Hecke character $\Psi$ such that $\Psi(\widetilde{a})=\psi\left(\widetilde{a}_{\mathcal{N}}\right) \psi_{\infty}(\widetilde{a})$ for all $\widetilde{a} \in K_{\infty}^{\times} \prod_{\mathfrak{p}}^{\infty} \mathcal{O}_{\mathfrak{p}}^{\times}$. There are only a finite number of such characters; in particular, if $\Psi$ and $\Phi$ are two such Hecke characters, then $\Psi \bar{\Phi}$ is a character on the $\mathcal{N}$-ideal class group (see [14]). By a Hecke character extending $\psi$ we shall mean a Hecke character extending $\psi \operatorname{sgn}(*)^{k}$ (i.e. $\psi \psi$ with $m=0$ ) in the above sense. If $\mathfrak{P}_{\infty}$ denotes the $K$-modulus consisting of the product of all the infinite primes of $K$, then it is clear that any Hecke character extending $\psi \psi_{\infty}$ has conductor dividing $\mathcal{N} \mathfrak{P}_{\infty}$. Consequently, given $\Psi$, we may define an ideal character $\Psi^{*}$ modulo $\mathcal{N} \mathfrak{P}_{\infty}$ by:

$$
\begin{cases}\Psi^{*}(\mathfrak{p})=\Psi\left(\widetilde{\pi}_{\mathfrak{p}}\right) & \text { for } \mathfrak{p} \nmid \mathcal{N} \text { and } \tilde{\pi} \mathcal{O}=\mathfrak{p} \\ \Psi^{*}(\mathfrak{a})=0 & \text { if }(\mathfrak{a}, \mathcal{N}) \neq 1\end{cases}
$$

Observe that if $\widetilde{a} \in K_{A}^{\times}$with $(\widetilde{a} \mathcal{O}, \mathcal{N})=1$, then $\Psi(\widetilde{a})=\Psi^{*}(\widetilde{a} \mathcal{O}) \psi\left(\widetilde{a}_{\mathcal{N}}\right) \psi_{\infty}(\widetilde{a})$, so that in particular, $\Psi^{*}(\xi \mathcal{O}) \psi(\xi) \operatorname{sgn}(\xi)^{k}|\xi|^{2 i m}=1$ for all $\xi \in \mathcal{O}$ with $(\xi, \mathcal{N})=1$ (cf. $[11$, p. 650$])$. Also, $\Psi$ and $\Psi^{*}$ both have modulus 1 , and they have finite order iff $\psi_{\infty}$ has finite order.

If $\mathbf{f}=\left(f_{1}, \ldots, f_{h}\right) \in \mathfrak{M}_{k}(\mathcal{N}, \psi, m)$, then $f_{\lambda}$ has a Fourier expansion of the form

$$
f_{\lambda}(\tau)=a_{\lambda}(0)+\sum_{0 \ll \xi \in \mathcal{I}_{\lambda}} a_{\lambda}(\xi) \exp (2 \pi i \operatorname{tr}(\xi \tau))
$$

Following Shimura we define

$$
C(\mathfrak{m}, \mathbf{f})= \begin{cases}N(\mathfrak{m})^{k_{0} / 2} a_{\lambda}(\xi) \xi^{-k / 2} & \text { if } \mathfrak{m}=\xi \mathcal{I}_{\lambda}^{-1} \subset \mathcal{O} \\ 0 & \text { otherwise }\end{cases}
$$

where $k_{0}=\max \left\{k_{1}, \ldots, k_{n}\right\}$; we refer to the $C(\mathfrak{m}, \mathbf{f})$ as the Fourier coefficients of f. We use these Fourier coefficients to associate a Dirichlet series to f:

$$
D(s, \mathbf{f})=\sum_{\mathfrak{m} \subset \mathcal{O}} C(\mathfrak{m}, \mathbf{f}) N(\mathfrak{m})^{-s} .
$$


Furthermore, it is easy to describe the action of two important operators on $\mathcal{M}_{k}(\mathcal{N}, \Psi)$

— the Hecke operators and the shift operators - in terms of their action on Fourier coefficients (for complete definitions see [11]).

For functions on $G_{A}$, we first define an analog of the classical slash operator: if $\mathbf{f} \in \mathfrak{M}_{k}(\mathcal{N}, \psi, m)$ and $y \in G_{A}$, we define $\mathbf{f} \mid y(x)=\mathbf{f}\left(x y^{\iota}\right)$. Then for an integral ideal $\mathfrak{q}$, the shift operator $B_{\mathfrak{q}}$ is characterized as follows: Let $\widetilde{q} \in K_{A}^{\times}$with $\widetilde{q}_{\infty}=1$ and $\widetilde{q} \mathcal{O}=\mathfrak{q}$. Define $\mathbf{f}\left|B_{\mathfrak{q}}=N(\mathfrak{q})^{-k_{0} / 2} \mathbf{f}\right|\left(\begin{array}{cc}1 & 0 \\ 0 & \widetilde{q}^{-1}\end{array}\right)$. Then $B_{\mathfrak{q}} \operatorname{maps} \mathcal{M}_{k}(\mathcal{N}, \Psi)$ to $\mathcal{M}_{k}(\mathcal{N} \mathfrak{q}, \Psi)$, and if $\mathbf{f} \in \mathcal{M}_{k}(\mathcal{N}, \Psi)$ then $C\left(\mathfrak{m}, \mathbf{f} \mid B_{\mathfrak{q}}\right)=C\left(\mathfrak{m} \mathfrak{q}^{-1}, \mathbf{f}\right)$ where as always we understand that $C(\mathfrak{n}, \mathbf{f})=0$ if $\mathfrak{n}$ is not integral. Clearly, we have $\mathbf{f}\left|B_{\mathfrak{q}_{1}}\right| B_{\mathfrak{q}_{2}}=$ f $\mid B_{\mathfrak{q}_{1} \mathfrak{q}_{2}}$.

For an integral ideal $\mathfrak{n}$, the Hecke operator $T_{\mathfrak{n}}=T_{\mathfrak{n}}^{\mathcal{N}} \operatorname{maps} \mathcal{M}_{k}(\mathcal{N}, \Psi)$ to $\mathcal{M}_{k}(\mathcal{N}, \Psi)$, independent of whether $(\mathfrak{n}, \mathcal{N})=1$. On Fourier coefficients, the action is

$$
C\left(\mathfrak{m}, \mathbf{f} \mid T_{\mathcal{N}}(\mathfrak{n})\right)=\sum_{\mathfrak{m}+\mathfrak{n} \subset \mathfrak{a}} \Psi^{*}(\mathfrak{a}) N(\mathfrak{a})^{k_{0}-1} C\left(\mathfrak{a}^{-2} \mathfrak{m} \mathfrak{n}, \mathbf{f}\right)
$$

where $\Psi^{*}$ is the ideal character defined on ideals prime to $\mathcal{N}$ induced from the Hecke character $\Psi$ and extended to all ideals as described earlier. In particular, even if $\Psi$ is the trivial character, $\Psi^{*}$ has the property that $\Psi^{*}(\mathfrak{a})=0$ for $(\mathfrak{a}, \mathcal{N}) \neq 1$. Both $B_{\mathfrak{q}}$ and $T_{\mathfrak{n}}$ take cusp forms to cusp forms.

\section{THE $W_{\mathcal{Q}}$ OPERATOR: DEFINITION AND BASIC PROPERTIES.}

In this section we define and give the basic properties of the $W_{\mathcal{Q}}$ operator which is critical to our development of the theory of newforms and to our investigation of character twists of newforms.

Fix a space $\mathcal{M}_{k}(\mathcal{N}, \Psi) \subset \mathfrak{M}_{k}(\mathcal{N}, \psi, m)$, where $\Psi$ is a Hecke character extending $\psi \psi_{\infty}$. In the absense of comments to the contrary, we take $\mathcal{Q}$ and $\mathcal{M}$ to denote relatively prime integral ideals with $\mathcal{N}=\mathcal{Q M}$, and we write $\psi=\psi_{\mathcal{Q}} \psi_{\mathcal{M}}$ where $\psi_{\mathcal{Q}}$ and $\psi_{\mathcal{M}}$ denote numerical chararacters modulo $\mathcal{Q}$ and $\mathcal{M}$ respectively. Then somewhat tedious but routine computations give us Propositions $2.1-2.7$; these are essentially straightforward generalizations of Propositions $1.1-1.5$ of [1] and Lemmas $1-4$ of [5]. The first of these is

Proposition 2.1. Let $\mathfrak{q}$ be a prime and suppose that $\mathfrak{q}^{2} \mid \mathcal{N}$ and $\psi$ is a character modulo $\mathcal{N q}^{-1}$. Then $T_{\mathfrak{q}}$ maps $\mathcal{M}_{k}(\mathcal{N}, \Psi)$ to $\mathcal{M}_{k}\left(\mathcal{N q}^{-1}, \Psi\right)$, and hence maps $\mathfrak{M}_{k}(\mathcal{N}, \psi, m)$ to $\mathfrak{M}_{k}\left(\mathcal{N q}^{-1}, \psi, m\right)$

Our $W_{\mathcal{Q}}$ operator is a generalization of Li's operator $V_{Q}$ and Atkin and Li's operator $W_{Q}$, however in the Hilbert modular case, it depends not only upon the numerical character of the space but also upon the choice of Hecke character extending the numerical character. We define it as follows.

Definition. Let $\Psi_{\mathcal{Q}}$ be a Hecke character extending $\psi_{\mathcal{Q}}\left(\right.$ i.e. $\left.\psi_{\mathcal{Q}} \operatorname{sgn}(*)^{k}\right)$. Choose $y \in G_{A}$ so that $y_{\infty}=1,(\operatorname{det} y) \mathcal{O}=\mathcal{Q}$, and $y_{0}=\left(\begin{array}{l}\tilde{a} \widetilde{b} \\ \widetilde{c} \\ d\end{array}\right)_{0}$ with $\widetilde{a} \mathcal{O}, \tilde{d} \mathcal{O} \subseteq \mathcal{Q}$; so $\widetilde{b} \mathcal{O} \subseteq \mathfrak{d}^{-1}$ and $\widetilde{c} \mathcal{O} \subseteq \mathcal{N} \mathfrak{d}$ (recall that $y_{0}$ is the finite part of $y$ ). For $x \in G_{A}$, define $W_{\mathcal{Q}}\left(\Psi_{\mathcal{Q}}\right)$ by:

$$
\mathbf{f} \mid W_{\mathcal{Q}}\left(\Psi_{\mathcal{Q}}\right)(x)=\bar{\Psi}_{\mathcal{Q}}(\operatorname{det} x) \overline{\psi_{\mathcal{Q}}}\left(\widetilde{b} \tilde{t}_{\mathfrak{d}} \bmod \mathcal{Q}\right) \overline{\psi_{\mathcal{M}}}(\widetilde{a} \bmod \mathcal{M})(\mathbf{f} \mid y)(x) .
$$


To specify the level, $W_{\mathcal{Q}}$ is sometimes denoted $W_{\mathcal{Q}}^{\mathcal{N}}$.

Remark. In [2], Flath has given a definition of a $W_{\mathcal{Q}}$-operator defined on the $\mathbb{Q}$-ideles which is essentially the same as our $W_{\mathcal{Q}}$-operator when $K=\mathbb{Q}$.

We can now state Propositions $2.2-2.7$.

Proposition 2.2. Let the notation be as above with $\mathcal{N}=\mathcal{Q M}$ where $\mathcal{Q}$ and $\mathcal{M}$ are relatively prime integral ideals and $\psi=\psi_{\mathcal{Q}} \psi_{\mathcal{M}}$. Let $\Psi$ be a Hecke character extending $\psi \psi_{\infty}$ and $\Psi_{\mathcal{Q}}$ a Hecke character extending $\psi_{\mathcal{Q}}$. Then $W_{\mathcal{Q}}\left(\Psi_{\mathcal{Q}}\right)$ takes $\mathcal{M}_{k}(\mathcal{N}, \Psi)$ to $\mathcal{M}_{k}\left(\mathcal{N}, \Psi \bar{\Psi}_{\mathcal{Q}}^{2}\right)$, and hence takes $\mathfrak{M}_{k}(\mathcal{N}, \psi, m)$ to $\mathfrak{M}_{k}\left(\mathcal{N}, \psi \bar{\psi}_{\mathcal{Q}}^{2}, m\right)$. Moreover, if $\mathbf{f} \in$ $\mathcal{M}_{k}(\mathcal{N}, \Psi)$

$\mathbf{f}\left|W_{\mathcal{Q}}\left(\Psi_{\mathcal{Q}}\right)\right| W_{\mathcal{Q}}\left(\bar{\Psi}_{\mathcal{Q}}\right)=\psi_{\mathcal{Q}}(-1) \mathbf{f}$.

then

Proposition 2.3. If $\mathcal{L}$ is an integral ideal, and $\mathcal{Q} \mid \mathcal{N} \mathcal{L}$ with $\left(\mathcal{Q}, \mathcal{N} \mathcal{L} \mathcal{Q}^{-1}\right)=1$, and $\Psi_{\mathcal{Q}}$ is a Hecke character extending $\psi_{\mathcal{Q}}$, then for $\mathbf{f} \in \mathcal{M}_{k}(\mathcal{N}, \Psi) \subset \mathfrak{M}_{k}(\mathcal{N}, \psi, m)$, we have

$$
\mathbf{f}\left|B_{\mathcal{L}}\right| W_{\mathcal{Q}}^{\mathcal{N} \mathcal{L}}\left(\Psi_{\mathcal{Q}}\right)= \begin{cases}\Psi_{\mathcal{Q}}^{*}(\mathcal{L}) \mathbf{f}\left|W_{\mathcal{Q}}^{\mathcal{N}}\left(\Psi_{\mathcal{Q}}\right)\right| B_{\mathcal{L}} & \text { if }(\mathcal{Q}, \mathcal{L})=1 \\ N(\mathcal{L})^{-k_{0} / 2} \mathbf{f} \mid W_{\mathcal{Q} \mathcal{L}^{-1}}^{\mathcal{N}}\left(\Psi_{\mathcal{Q}}\right) & \text { if } \mathcal{L} \mid \mathcal{Q}\end{cases}
$$

Proposition 2.4. Let $\mathfrak{p}$ be a prime with $\mathfrak{p} \nmid \mathcal{Q}$, and let $\Psi_{\mathcal{Q}}$ be a Hecke character extending $\psi_{\mathcal{Q}}$. For $\mathbf{f} \in \mathcal{M}_{k}(\mathcal{N}, \Psi)$ we have

$$
\mathbf{f}\left|W_{\mathcal{Q}}\left(\Psi_{\mathcal{Q}}\right)\right| T_{\mathfrak{p}}=\bar{\Psi}_{\mathcal{Q}}^{*}(\mathfrak{p}) \mathbf{f}\left|T_{\mathfrak{p}}\right| W_{\mathcal{Q}}\left(\Psi_{\mathcal{Q}}\right)
$$

Proposition 2.5. Suppose that $\mathfrak{q}$ is a prime such that $\mathfrak{q} \mid \mathcal{N}$, but $\mathfrak{q}^{2} \nmid \mathcal{N}$, and that $\psi$ is a character modulo $\mathcal{N q}^{-1}$. Let $\Psi$ be a Hecke character extending $\psi \psi_{\infty}$. Then $T_{\mathfrak{q}}+N(\mathfrak{q})^{k_{0} / 2-1} W_{\mathfrak{q}}$ maps $\mathcal{M}_{k}(\mathcal{N}, \Psi)$ to $\mathcal{M}_{k}\left(\mathcal{N}^{-1}, \Psi\right)$.

Remark. Here $W_{\mathfrak{q}}=W_{\mathcal{Q}}(1)$ since $\psi_{\mathfrak{q}}=1$.

Proposition 2.6. Suppose $\mathbf{f}=\mathbf{g} \mid B_{\mathfrak{q}} \in \mathcal{S}_{k}(\mathcal{N}, \Psi)$ for some prime $\mathfrak{q}$. If $\mathfrak{q} \mid \mathcal{N}$ and $\psi$ is a character modulo $\mathcal{N} \mathfrak{q}^{-1}$, then $\mathbf{g} \in \mathcal{S}_{k}\left(\mathcal{N} \mathfrak{q}^{-1}, \Psi\right)$. If $\mathfrak{q} \nmid \mathcal{N}$ then $\mathbf{f}=0$.

Proposition 2.7. Let $\mathcal{Q}, \mathcal{Q}^{\prime}$ be divisors of $\mathcal{N}$ such that $\left(\mathcal{Q}, \mathcal{N} \mathcal{Q}^{-1}\right)=1$ and $\left(\mathcal{Q}^{\prime}, \mathcal{Q N} \mathcal{Q}^{\prime-1}\right)=1$, and let $\mathbf{f} \in \mathcal{M}_{k}(\mathcal{N}, \Psi) \subset \mathfrak{M}_{k}(\mathcal{N}, \psi, m)$. Let $\psi_{\mathcal{Q}}\left(\right.$ resp. $\left.\psi_{\mathcal{Q}^{\prime}}\right)$ denote the $\mathcal{Q}$ - (resp. $\mathcal{Q}^{\prime}$-) part of $\psi$ and let $\Psi_{\mathcal{Q}}$ (resp. $\Psi_{\mathcal{Q}^{\prime}}$ ) be Hecke characters which extend the corresponding numerical characters. Then

$$
\mathbf{f}\left|W_{\mathcal{Q}}\left(\Psi_{\mathcal{Q}}\right)\right| W_{\mathcal{Q}^{\prime}}\left(\Psi_{\mathcal{Q}^{\prime}}\right)=\bar{\Psi}_{\mathcal{Q}}^{*}\left(\mathcal{Q}^{\prime}\right) \mathbf{f} \mid W_{\mathcal{Q} \mathcal{Q}^{\prime}}\left(\Psi_{\mathcal{Q}} \Psi_{\mathcal{Q}^{\prime}}\right)
$$

\section{NeWFORMS IN $\mathcal{S}_{k}(\mathcal{N}, \Psi)$}

Now that we have developed analogs of the operators defined in [5] and [1]] and we have established some preliminary propositions describing their interactions (Propositions $2.1-2.7$ ), many of the theorems in [5] have natural generalizations to the Hilbert modular case. In this section, we give a summary of the theory of Hilbert modular newforms which culminates in a theorem characterizing newforms as cusp forms whose associated Dirichlet series has a prescribed Euler product and 
which are eigenfunctions for a certain involution (Theorem 3.7). In Theorem 3.3 we characterize the Hecke eigenvalues for primes dividing the level.

Given $\mathcal{S}_{k}(\mathcal{N}, \Psi) \subset \mathfrak{S}_{k}(\mathcal{N}, \psi, m)$, let $\mathcal{S}_{k}^{-}(\mathcal{N}, \Psi)$ be the subspace of $\mathcal{S}_{k}(\mathcal{N}, \Psi)$ generated by all $\mathbf{g} \mid B_{\mathcal{Q}}$ where $\mathbf{g} \in \mathcal{S}_{k}\left(\mathcal{N}^{\prime}, \Psi\right) \subset \mathfrak{S}_{k}\left(\mathcal{N}^{\prime}, \psi, m\right)$ with $\mathcal{N}^{\prime} \mid \mathcal{N}, \mathcal{N}^{\prime} \neq \mathcal{N}$, and $\mathcal{Q N}^{\prime} \mid \mathcal{N}$. As in the elliptic modular case, it is easy to see that $\mathcal{S}_{k}^{-}(\mathcal{N}, \Psi)$ is invariant under the action of the Hecke operators $T_{\mathfrak{n}}$ with $(\mathfrak{n}, \mathcal{N})=1$. With this notation, the proof of Theorem 3.1 below follows in analogy to the proof in [5] for the case of $K=\mathbb{Q}$; since no significantly new ideas are required to prove this generalization, we state it without proof.

Theorem 3.1. If $\mathbf{f} \in \mathcal{S}_{k}(\mathcal{N}, \Psi)$ has the property that $C(\mathfrak{m}, \mathbf{f})=0$ if $(\mathfrak{m}, \mathfrak{a})=1$ where $\mathfrak{a}$ is a fixed integral ideal, then $\mathbf{f} \in \mathcal{S}_{k}^{-}(\mathcal{N}, \Psi)$.

Shimura defines $((2.28)$ of [11]) a Petersson inner product $\langle\mathbf{f}, \mathbf{g}\rangle$ for $\mathbf{f}, \mathbf{g} \in$ $\mathcal{S}_{k}(\mathcal{N}, \Psi)$. If $\mathbf{f}=\left(f_{1}, \ldots, f_{h}\right)$ and $\mathbf{g}=\left(g_{1}, \ldots, g_{h}\right)$, then $\langle\mathbf{f}, \mathbf{g}\rangle=\sum_{\lambda=1}^{h}\left\langle f_{\lambda}, g_{\lambda}\right\rangle$ where $\left\langle f_{\lambda}, g_{\lambda}\right\rangle$ is given by (2.27) of [11]. A standard argument shows that for a matrix $A \in G L_{2}^{+}(K),\left\langle f_{\lambda}\left|A, g_{\lambda}\right| A\right\rangle=\left\langle f_{\lambda}, g_{\lambda}\right\rangle$ (see e.g. (3.4) of [13]). This invariance extends in an obvious way to $\langle\mathbf{f}, \mathbf{g}\rangle:$ Let $\mathbf{f}=\left(f_{1}, \ldots, f_{h}\right), \mathbf{g}=\left(g_{1}, \ldots, g_{h}\right) \in \mathcal{S}_{k}(\mathcal{N}, \Psi)$, $T$ be an operator which maps $\mathcal{S}_{k}(\mathcal{N}, \Psi)$ to $\mathcal{S}_{k}(\mathcal{M}, \Phi)$, and put $\mathbf{f}^{\prime}=\left(f_{1}^{\prime}, \ldots, f_{h}\right), \mathbf{g}^{\prime}=$ $\left(g_{1}^{\prime}, \ldots, g_{h}^{\prime}\right) \in \mathcal{S}_{k}(\mathcal{M}, \Phi)$, where $\mathbf{f} \mid T=\mathbf{f}^{\prime}$ and $\mathbf{g} \mid T=\mathbf{g}^{\prime}$. If for each index $\mu$ there exists an index $\lambda$ and a $A_{\lambda} \in G L_{2}^{+}(K)$ such that $f_{\mu}^{\prime}=f_{\lambda} \mid A_{\lambda}$ and $g_{\mu}^{\prime}=g_{\lambda} \mid A_{\lambda}$, then $\langle\mathbf{f}|T, \mathbf{g}| T\rangle=\langle\mathbf{f}, \mathbf{g}\rangle$. This will be the case with most of the operators we define.

With respect to this inner product defined above, the Hecke operators are essentially hermitian (Proposition 2.4 of [11]):

$$
\Psi^{*}(\mathfrak{m})\left\langle\mathbf{f} \mid T_{\mathfrak{m}}, \mathbf{g}\right\rangle=\left\langle\mathbf{f}, \mathbf{g} \mid T_{\mathfrak{m}}\right\rangle
$$

for all integral ideals $(\mathfrak{m}, \mathcal{N})=1$. Let $\mathcal{S}_{k}^{+}(\mathcal{N}, \Psi)$ denote the orthogonal complement of $\mathcal{S}_{k}^{-}(\mathcal{N}, \Psi)$ in $\mathcal{S}_{k}(\mathcal{N}, \Psi)$. It follows from the invariance of $\mathcal{S}_{k}^{-}(\mathcal{N}, \Psi)$ under the Hecke operators and the hermitian property of the Hecke operators that $\mathcal{S}_{k}^{+}(\mathcal{N}, \Psi)$ is also invariant under all Hecke operators $T_{\mathfrak{n}}$ where $(\mathfrak{n}, \mathcal{N})=1$.

Definition. A newform $\mathbf{f} \in \mathcal{S}_{k}(\mathcal{N}, \Psi)$ is a form in $\mathcal{S}_{k}^{+}(\mathcal{N}, \Psi)$ which is a simultaneous eigenform for all Hecke operators $T_{\mathfrak{p}}$ with $\mathfrak{p}$ a prime, $\mathfrak{p} \nmid \mathcal{N}$. The form is normalized if $C(\mathcal{O}, \mathbf{f})=1$.

As in the classical case, if $\mathbf{f}$ is a newform in $\mathcal{S}_{k}(\mathcal{N}, \Psi)$ and $\mathbf{f} \mid T_{\mathfrak{p}}=\lambda_{\mathfrak{p}} \mathbf{f}$ then $C(\mathfrak{p}, \mathbf{f})=\lambda_{\mathfrak{p}} C(\mathcal{O}, \mathbf{f})$ for all $\mathfrak{p} \nmid \mathcal{N}$. It follows from Theorem 1 that $C(\mathcal{O}, \mathbf{f}) \neq 0$, and hence $\mathbf{f}$ can be normalized. Moreover, if $\mathbf{f}, \mathbf{g} \in \mathcal{S}_{k}^{+}(\mathcal{N}, \Psi)$ are both newforms with the same eignevalues for all $T_{\mathfrak{p}}$ for $\mathfrak{p} \nmid \mathcal{N}$ then by Theorem 3.1, we have $\mathbf{f}-\mathbf{g} \in$ $\mathcal{S}_{k}^{-}(\mathcal{N}, \Psi)$, hence $\mathbf{f}$ is uniquely determined up to a scalar multiple by its eigenvalues. (From a representation-theoretic point of view, this result is due to Miyake [7] although the results there are in a different context which is difficult to compare to our own.) Since $\left\{T_{\mathfrak{p}} \mid \mathfrak{p} \nmid \mathcal{N}\right\}$ is a commuting family of hermitian operators, $\mathcal{S}_{k}^{+}(\mathcal{N}, \Psi)$ has an orthogonal basis consisting of newforms. A form $\mathbf{g} \in \mathcal{S}_{k}^{-}(\mathcal{N}, \Psi)$ is an oldform if $\mathbf{g}=\mathbf{h} \mid B_{\mathcal{Q}}$ for some newform $\mathbf{h} \in \mathcal{S}_{k}\left(\mathcal{N}^{\prime}, \Psi\right) \subset \mathfrak{S}_{k}\left(\mathcal{N}^{\prime}, \psi, m\right)$ with $\mathcal{Q N}^{\prime} \mid \mathcal{N}$. It is easy to see that $\mathcal{S}_{k}^{-}(\mathcal{N}, \Psi)$ is generated by the oldforms in $\mathcal{S}_{k}^{-}(\mathcal{N}, \Psi)$, and if $\mathbf{g} \in \mathcal{S}_{k}^{-}(\mathcal{N}, \Psi)$ is a simultaneous eigenform for all $T_{\mathfrak{p}}, \mathfrak{p} \nmid \mathcal{N}$, then there exists a newform $\mathbf{h} \in \mathcal{S}_{k}^{+}\left(\mathcal{N}^{\prime}, \Psi\right) \subset \mathfrak{S}_{k}\left(\mathcal{N}^{\prime}, \psi, m\right)$ with $\mathcal{N}^{\prime} \mid \mathcal{N}$ having the same eigenvalues as $\mathbf{g}$ for all such $T_{\mathfrak{p}}$. In fact, we have the following result which is crucial to a number of arguments in this paper (e.g. Theorem 3.6). 
Proposition 3.2. Let $\mathbf{g} \in \mathcal{S}_{k}(\mathcal{M}, \Psi)$ be a newform, and suppose that $\mathcal{M} \mid \mathcal{N}$. Then $\left\{\mathbf{g}\left|B_{\mathfrak{a}}: \mathfrak{a}\right| \mathcal{N} \mathcal{M}^{-1}\right\}$ is linearly independent.

Proof. Clearly, $\sum_{\mathfrak{a} \mid \mathcal{N} \mathcal{M}^{-1}} c_{\mathfrak{a}} \mathbf{g} \mid B_{\mathfrak{a}}=0$ if and only if $\sum_{\mathfrak{a} \mid \mathcal{N \mathcal { M }}-1} c_{\mathfrak{a}} C\left(\mathfrak{m a}^{-1}, \mathbf{g}\right)=0$ for all integral ideals $\mathfrak{m}$. The idea then is to choose an ideal $\mathfrak{m}$ for which $C\left(\mathfrak{m a} \mathfrak{a}^{-1}, \mathbf{g}\right) \neq 0$ for some $\mathfrak{a}$ occuring in the sum, but for which $\mathfrak{m b}^{-1}$ is not integral (and hence $C\left(\mathfrak{m} \mathfrak{b}^{-1}, \mathbf{g}\right)=0$ ) for any other ideal $\mathfrak{b}$ occuring in the sum. This forces $c_{\mathfrak{a}}=0$, and the result follows by induction. Since the only coefficient of $\mathbf{g}$ that we know is nonzero is $C(\mathcal{O}, \mathbf{g})$, the choice of $\mathfrak{m}$ is clear.

Suppose $\sum_{\mathfrak{a} \mid \mathcal{N M}^{-1}} c_{\mathfrak{a}} \mathbf{g} \mid B_{\mathfrak{a}}=0$; fix an ideal $\mathfrak{a}$ of minimal (absolute) norm, and put $\mathfrak{m}=\mathfrak{a}$. Then

$$
\sum_{\mathfrak{a} \mid \mathcal{N} \mathcal{M}^{-1}} c_{\mathfrak{a}} C\left(\mathfrak{m a \mathfrak { a }} \mathfrak{a}^{-1}, \mathbf{g}\right)=\sum_{\substack{\mathfrak{b} \mid \mathcal{N} \mathcal{M}^{-1} \\ N(\mathfrak{b})=N(\mathfrak{a})}} c_{\mathfrak{b}} C\left(\mathfrak{a} \mathfrak{b}^{-1}, \mathbf{g}\right)+\sum_{\substack{\mathfrak{b} \mid \mathcal{N M}^{-1} \\ N(\mathfrak{b})>N(\mathfrak{a})}} c_{\mathfrak{b}} C\left(\mathfrak{a} \mathfrak{b}^{-1}, \mathbf{g}\right) .
$$

If $N(\mathfrak{b})>N(\mathfrak{a})$ then clearly $\mathfrak{a b}^{-1}$ is not integral, so the second sum vanishes. So suppose $N(\mathfrak{a})=N(\mathfrak{b})$; then $\mathfrak{a}$ and $\mathfrak{b}$ lie above the same primes of $\mathbb{Q}$. Write $\mathfrak{a}=\mathfrak{p}_{1}^{r_{1}} \cdots \mathfrak{p}_{s}^{r_{s}}$ and $\mathfrak{b}=\mathfrak{p}_{1}^{r_{1}^{\prime}} \cdots \mathfrak{p}_{s}^{r_{s}^{\prime}}$ where the $\mathfrak{p}_{j}$ are primes of $K$. With $\mathfrak{m}=\mathfrak{a}$ fixed we see that $\mathfrak{m b} \mathfrak{b}^{-1}=\mathfrak{a b}^{-1} \subset \mathcal{O}$ if and only if $\mathfrak{b} \mid \mathfrak{a}$ and hence if and only if $r_{j}^{\prime} \leq r_{j}$ for $1 \leq j \leq s$. If $\mathfrak{b} \neq \mathfrak{a}$ then $r_{k}^{\prime}<r_{k}$ for some $k$, hence $N(\mathfrak{b})<N(\mathfrak{a})$, contrary to assumption. Thus only one term survives in the first summand as desired.

Now we come to an important theorem about newforms which generalizes Theorem 3 of [5].

Theorem 3.3. Let $\mathbf{f}$ be a normalized newform in $\mathcal{S}_{k}(\mathcal{N}, \Psi)$, and $\mathfrak{p}, \mathfrak{q}$ primes with $\mathfrak{p} \nmid \mathcal{N}$ and $\mathfrak{q} \mid \mathcal{N}$.

(1) The Dirichlet series attached to $\mathbf{f}, D(s, \mathbf{f})=\sum_{\mathfrak{m} \subset \mathcal{O}} C(\mathfrak{m}, \mathbf{f}) N(\mathfrak{m})^{-s}$ has the Euler product

$$
\begin{aligned}
D(s, \mathbf{f})= & \prod_{\mathfrak{q} \mid \mathcal{N}}\left(1-C(\mathfrak{q}, \mathbf{f}) N(\mathfrak{q})^{-s}\right)^{-1} \\
& \prod_{\mathfrak{p} \nmid \mathcal{N}}\left(1-C(\mathfrak{p}, \mathbf{f}) N(\mathfrak{p})^{-s}+\Psi^{*}(\mathfrak{p}) N(\mathfrak{p})^{k_{0}-1-2 s}\right)^{-1}
\end{aligned}
$$

(2) If $\psi$ is not defined modulo $\mathcal{N q}^{-1}$, then either $C(\mathfrak{q}, \mathbf{f})=0$ or $|C(\mathfrak{q}, \mathbf{f})|=$ $N(\mathfrak{q})^{\left(k_{0}-1\right) / 2}$. Moreover, $C(\mathfrak{q}, \mathbf{f}) \neq 0$ whenever the inertial degree of $\mathfrak{q}$ (over Q) is 1 , or when $\mathfrak{q} \| \mathcal{N}$.

(3) If $\psi$ is a character modulo $\mathcal{N q}^{-1}$, then $C(\mathfrak{q}, \mathbf{f})=0$ if $\mathfrak{q}^{2} \mid \mathcal{N}$ and $C(\mathfrak{q}, \mathbf{f})^{2}=$ $N(\mathfrak{q})^{k_{0}-2}$ if $\mathfrak{q}^{2} \nmid \mathcal{N}$.

Remark. The referee informs us that it follows from the representation theory that in part (2) of Theorem 3.3 the coefficient $C(\mathfrak{q}, \mathbf{f})$ is never zero. We are not able to verify this from our classical point of view however, the stated condition is not overly restrictive since the set of primes having inertial degree one over $\mathbb{Q}$ has density one (see $[4$, p. 131]). Moreover, if $K / \mathbb{Q}$ is an abelian extension, then class 
field theory tells us that the rational primes which split completely are described by congruence conditions in $\mathbb{Q}$, so we can explicitly compute levels at which $C(\mathfrak{q}, \mathbf{f}) \neq 0$. Nonetheless, it would be interesting to have a classical proof that $C(\mathfrak{q}, \mathbf{f}) \neq 0$ without restrictions on the prime $\mathfrak{q}$ when $\psi$ is not defined modulo $\mathcal{N q}^{-1}$. The issue of nonvanishing of this coefficient represents far more than idle curiosity for as Theorem 5.8 suggests, whether $C(\mathfrak{q}, \mathbf{f})$ vanishes is at the heart of the question of whether the twist of a newform $\mathbf{f}$ is again a newform.

Proof. The proofs of parts (1) and (3) are analogous to the proofs in [5]. For part (2), one must generalize Theorems 3 and 4 of [8]; here we find that Ogg's proof of Theorem 3 is valid only for primes of degree one. In this context, the generalizations are straightforward.

To proceed, we need several operators: Let $\mathbf{f} \in \mathcal{M}_{k}(\mathcal{N}, \Psi) \subset \mathfrak{M}_{k}(\mathcal{N}, \psi, m)$ and suppose that $\psi$ is a character modulo $\mathcal{M}$ where $\mathcal{M} \mid \mathcal{N}$. Choose $w_{j} \in W(\mathcal{M})$ such that $\left(w_{j}\right)_{\infty}=1$ and

$$
W(\mathcal{M})=\bigcup_{j} W(\mathcal{N}) w_{j} \quad(\text { disjoint })
$$

define the trace operator $\operatorname{Tr}_{\mathcal{M}}^{\mathcal{N}}$ by

$$
\mathbf{f}\left|\operatorname{Tr}_{\mathcal{M}}^{\mathcal{N}}=\sum_{j} \overline{\psi_{Y}}\left(w_{j}\right) \mathbf{f}\right| w_{j}
$$

Notice that this expression is clearly well-defined, and if $\left\{A_{j}\right\}$ is a complete set of coset representatives for $\Gamma_{\lambda}(\mathcal{M}) \backslash \Gamma_{\lambda}(\mathcal{N})$, then we may take $\left(w_{j}\right)_{0}=x_{\lambda}^{\iota} A_{j} x_{\lambda}^{-\iota}=$ $x_{\lambda}^{-1} A_{j} x_{\lambda}$. It is straightforward to check that $\mathbf{f} \mid \operatorname{Tr}_{\mathcal{M}}^{\mathcal{N}} \in \mathcal{M}_{k}(\mathcal{M}, \Psi)$.

The conjugation operator, $K$, can be defined by its action on Fourier coefficients: $C(\mathfrak{m}, \mathbf{f} \mid K)=\overline{C(\mathfrak{m}, \mathbf{f})}$ (where the bar denotes complex conjugation), or by its action on the components of $\mathbf{f}$ : if $\mathbf{f}=\left(f_{1}, \ldots, f_{h}\right)$, then $\mathbf{f} \mid K=\left(g_{1}, \ldots, g_{h}\right)$ where $g_{j}(z)=\overline{f_{j}(-\bar{z})}$ (this operator is defined in [11] and denoted there as $\mathbf{f}^{\rho}$ ).

Now we define the operator $H_{\mathcal{N}}$ (the "canonical involution"); this is essentially Shimura's operator $J_{\mathcal{N}}$, although we rescale it here for consistency with the operator $W_{\mathcal{Q}}$ when $\mathcal{Q}=\mathcal{N}$. Let $\tilde{n} \in K_{A}^{\times}$with $\widetilde{n} \mathcal{O}=\mathcal{N}$, and put $y_{0}=\left(\begin{array}{cc}0 & \widetilde{t}_{\mathfrak{d}}^{-1} \\ \tilde{n} \tilde{t}_{\mathfrak{d}} & 0\end{array}\right)_{0}$ with $y_{\infty}=1$; define $\left(\mathbf{f} \mid H_{\mathcal{N}}\right)(x)=(\mathbf{f} \mid y)\left(x^{-\iota}\right)$. For $\mathbf{f} \in \mathcal{M}_{k}(\mathcal{N}, \Psi)$, we have $\mathbf{f} \mid H_{\mathcal{N}}=$ $(-1)^{k} \bar{\Psi}\left(\widetilde{t}_{\mathfrak{d}}\right) \mathbf{f} \mid J_{\mathcal{N}}$.

From [11] (pp $653-655)$, we see that both $K$ and $H_{\mathcal{N}} \operatorname{map} \mathcal{M}_{k}(\mathcal{N}, \Psi) \subset$ $\mathfrak{M}_{k}(\mathcal{N}, \psi, m)$ to $\mathcal{M}_{k}(\mathcal{N}, \bar{\Psi}) \subset \mathfrak{M}_{k}(\mathcal{N}, \bar{\psi},-m)$, that $K \operatorname{maps} \mathcal{S}_{k}^{-}(\mathcal{N}, \Psi)$ to $\mathcal{S}_{k}^{-}(\mathcal{N}, \bar{\Psi})$ and $\mathcal{S}_{k}^{+}(\mathcal{N}, \Psi)$ to $\mathcal{S}_{k}^{+}(\mathcal{N}, \bar{\Psi})$, that $\mathbf{f}\left|T_{\mathfrak{p}}\right| K=\mathbf{f}|K| T_{\mathfrak{p}}$, and that $\mathbf{f}\left|H_{\mathcal{N}}\right| T_{\mathfrak{m}}=$ $\bar{\Psi}^{*}(\mathfrak{m}) \mathbf{f}\left|T_{\mathfrak{m}}\right| H_{\mathcal{N}}$ for all $(\mathfrak{m}, \mathcal{N})=1$. It is trivial to check that for $\mathbf{f} \in \mathcal{M}_{k}(\mathcal{N}, \Psi)$, $\mathbf{f}\left|H_{\mathcal{N}}\right| H_{\mathcal{N}}=(-1)^{k} \mathbf{f}, \mathbf{f}|K| K=\mathbf{f}, \mathbf{f}\left|B_{\mathfrak{a}}\right| K=\mathbf{f}|K| B_{\mathfrak{a}}$, and that $\mathbf{f}\left|H_{\mathcal{N}}\right| K=$ $(-1)^{k} \mathbf{f}|K| H_{\mathcal{N}}$. Also, one can easily show that if $\mathbf{g} \in \mathcal{S}_{k}(\mathcal{M}, \Psi)$ with $\mathcal{M} \mid \mathcal{N}$ and $\mathfrak{a}$ an integral ideal such that $\mathfrak{a} \mathcal{M} \mid \mathcal{N}$, then $\mathbf{g}\left|B_{\mathfrak{a}}\right| H_{\mathcal{N}}=N(\mathfrak{a})^{-k_{0}} N\left(\mathcal{N} \mathcal{M}^{-1}\right)^{k_{0} / 2} \mathbf{g}\left|H_{\mathcal{M}}\right| B_{\mathcal{N} \mathcal{M}^{-1} \mathfrak{a}^{-1}}$, so $H_{\mathcal{N}} \operatorname{maps} \mathcal{S}_{k}^{-}(\mathcal{N}, \Psi)$ to $\mathcal{S}_{k}^{-}(\mathcal{N}, \bar{\Psi})$. Moreover, since $\langle\mathbf{f}, \mathbf{g}\rangle=\left\langle\mathbf{f}\left|H_{\mathcal{N}}, \mathbf{g}\right| H_{\mathcal{N}}\right\rangle=$ $\sum_{\lambda=1}^{h}\left\langle f_{\lambda}\left|\beta_{\lambda}^{-1}, g_{\lambda}\right| \beta_{\lambda}^{-1}\right\rangle$ with $\beta_{\lambda} \in G L_{2}^{+}(K)$ (see (2.47) of [11]), it follows that $H_{\mathcal{N}} \operatorname{maps} \mathcal{S}_{k}^{+}(\mathcal{N}, \Psi)$ to $\mathcal{S}_{k}^{+}(\mathcal{N}, \bar{\Psi})$. Finally, from Proposition 2.10 of [11], we have 
that if $\mathbf{f} \in \mathcal{S}_{k}^{+}(\mathcal{N}, \Psi)$ is a newform then $\mathbf{f}\left|H_{\mathcal{N}}=\gamma \mathbf{f}\right| K$ for some constant $\gamma$ with $|\gamma|=1$.

As before, given these newly defined operators, it is now straightforward to obtain the following two generalizations of theorems in [5].

Theorem 3.4. Let $\mathbf{f} \in \mathcal{S}_{k}(\mathcal{N}, \Psi) \subset \mathfrak{S}_{k}(\mathcal{N}, \psi, m)$. Then $\mathbf{f} \in \mathcal{S}_{k}^{+}(\mathcal{N}, \Psi)$ if and only if for all primes $\mathfrak{q} \mid \mathcal{N}$ for which $\psi$ is defined modulo $\mathcal{N}^{-1}$, we have

$$
\mathbf{f}\left|\operatorname{Tr}_{\mathcal{N q}^{-1}}^{\mathcal{N}}=0=\mathbf{f}\right| H_{\mathcal{N}} \mid \operatorname{Tr}_{\mathcal{N q}^{-1}}^{\mathcal{N}}
$$

Theorem 3.5. Let $\mathbf{f}$, $\mathbf{g}$ be normalized newforms in $\mathcal{S}_{k}(\mathcal{N}, \Psi), \mathcal{S}_{k}(\mathcal{M}, \Psi)$ respectively such that $C(\mathfrak{p}, \mathbf{f})=C(\mathfrak{p}, \mathbf{g})$ for almost all primes $\mathfrak{p}$. Then $\mathbf{f}=\mathbf{g}$ and $\mathcal{M}=\mathcal{N}$.

We note here that while a knowledge of the eigenvalues $C(\mathfrak{p}, \mathbf{f})$ for almost all primes $\mathfrak{p}$ is sufficient to determine the level of a newform, it is not sufficient to determine the character. However, we can make the following statement.

Theorem 3.6. Let $\mathbf{f} \in \mathcal{S}_{k}(\mathcal{N}, \Psi) \subset \mathfrak{S}_{k}(\mathcal{N}, \psi, m)$, and $\mathbf{g} \in \mathcal{S}_{k}(\mathcal{M}, \Phi) \subset \mathfrak{S}_{k}\left(\mathcal{M}, \phi, m^{\prime}\right)$ be normalized newforms, and assume that they have the same eigenvalues for all the Hecke operators $T_{\mathfrak{m}}$ for $(\mathfrak{m}, \mathcal{M N})=1$. Then $\Psi=\Phi, \mathcal{M}=\mathcal{N}$, and $\mathbf{f}=\mathbf{g}$.

Proof. We need only show that $m=m^{\prime}$ and $\Psi=\Phi$; the result will then follow from the previous theorem.

Let $\mathfrak{p} \nmid \mathcal{M N}$ be a prime. Then

$$
\mathbf{f}\left|T_{\mathfrak{p}^{2}}=\mathbf{f}\right|\left(T_{\mathfrak{p}}\right)^{2}-N(\mathfrak{p})^{k_{0}-1} \mathbf{f} \mid S(\mathfrak{p})=\left[C(\mathfrak{p}, \mathbf{f})^{2}-N(\mathfrak{p})^{k_{0}-1} \Psi^{*}(\mathfrak{p})\right] \mathbf{f}
$$

(see [11] for the definition of $S(\mathfrak{p})$ ). Similarly, $\mathbf{g} \mid T_{\mathfrak{p}^{2}}=\left[C(\mathfrak{p}, \mathbf{g})^{2}-N(\mathfrak{p})^{k_{0}-1} \Phi^{*}(\mathfrak{p})\right] \mathbf{g}$. Since $C(\mathfrak{p}, \mathbf{f})=C(\mathfrak{p}, \mathbf{g})$, and $C(\mathfrak{p}, \mathbf{f})^{2}-N(\mathfrak{p})^{k_{0}-1} \Psi^{*}(\mathfrak{p})=C(\mathfrak{p}, \mathbf{g})^{2}-N(\mathfrak{p})^{k_{0}-1} \Phi^{*}(\mathfrak{p})$, we have $\Psi^{*}(\mathfrak{p})=\Phi^{*}(\mathfrak{p})$ for all $\mathfrak{p} \nmid \mathcal{M N}$. Thus $(\Psi \bar{\Phi})^{*}=1$

Let $S$ be the set of primes dividing $\mathcal{N M}$ together with the infinite primes of $K$, and let $K_{A, S}^{\times}$be the set of ideles which are 1 at all places in $S$. From above, we know that $\Psi \bar{\Phi}(\widetilde{a})=(\Psi \bar{\Phi})^{*}(\widetilde{a} \mathcal{O})=1$ for all ideles $\widetilde{a} \in K_{A, S}^{\times}$. Moreover, since $\Psi \bar{\Phi}$ is a Hecke character, it is trivial on $K^{\times}$. By Proposition VII.15 of [15], $K^{\times} K_{A, S}^{\times}$ is dense in $K_{A}^{\times}$, so by continuity of $\Psi \bar{\Phi}$, the Hecke character $\Psi \bar{\Phi}$ is trivial on all of $K_{A}^{\times}$; hence $\Phi=\Psi$.

Since $\psi_{\infty}(\widetilde{a})=\operatorname{sgn}\left(\widetilde{a}_{\infty}\right)^{k}\left|\widetilde{a}_{\infty}\right|^{2 i m}$ and $\phi_{\infty}(\widetilde{a})=\operatorname{sgn}\left(\widetilde{a}_{\infty}\right)^{k}\left|\widetilde{a}_{\infty}\right|^{2 i m^{\prime}}$, we have $(\psi \bar{\phi})_{\infty}(\widetilde{a})=$ $\left|\widetilde{a}_{\infty}\right|^{2 i r}$ where $r=m-m^{\prime} \in \mathbb{R}^{n}$; we claim that $r=0$. To see this, consider a sequence of ideles $\widetilde{a}_{\ell}$ with all components of $\widetilde{a}_{\ell}=1$ except for the $j^{\text {th }}$ infinite place. In that place of $\widetilde{a}_{\ell}$, let the value be $e^{\ell}$ ( $e$ the base for the natural logarithm). Then $1=\Psi \bar{\Phi}\left(\widetilde{a}_{\ell}\right)=(\psi \bar{\phi})_{\infty}\left(\widetilde{a}_{\ell}\right)=\left|e^{\ell}\right|^{2 i r_{j}}=e^{2 i \ell r_{j}}$. This implies that $r_{j} \in(\pi / \ell) \mathbb{Z}$ for all $\ell \geq 1$, hence $r_{j}=0$, which implies $m=m^{\prime}$ and so completes the proof.

The final result from [5] which we need to generalize is

Theorem 3.7. Suppose $\mathbf{f} \in \mathcal{S}_{k}(\mathcal{N}, \Psi) \subset \mathfrak{S}_{k}(\mathcal{N}, \psi, m)$, and $D(s, \mathbf{f})=\sum_{\mathcal{M} \subset \mathcal{O}} C(\mathfrak{m}, \mathbf{f}) N(\mathfrak{m})^{-s}$ is the Dirichlet series attached to $\mathbf{f}$. If $\mathbf{f}|K| H_{\mathcal{N}}=\gamma \mathbf{f}$ for some $\gamma \in \mathbb{C}^{\times}$and

$$
\begin{aligned}
D(s, \mathbf{f})= & \prod_{\mathfrak{q} \mid \mathcal{N}}\left(1-C(\mathfrak{q}, \mathbf{f}) N(\mathfrak{q})^{-s}\right)^{-1} \\
& \prod_{\mathfrak{p} \nmid \mathcal{N}}\left(1-C(\mathfrak{p}, \mathbf{f}) N(\mathfrak{p})^{-s}+\Psi^{*}(\mathfrak{p}) N(\mathfrak{p})^{k_{0}-1-2 s}\right)^{-1}
\end{aligned}
$$


then $\mathbf{f}$ is a normalized newform in $\mathcal{S}_{k}(\mathcal{N}, \Psi)$.

Proof. The proof of Theorem 3.7 is somewhat tedious, but follows exactly the line of argument in [5]. On the other hand, a few comments are in order. The proof relies in two places on Proposition 3.2, and requires a nontrivial estimate of the size of Fourier coefficients of cusp forms. The most general result to date is provided by [9]. Throughout the proof in the Hilbert modular case, the operator $W_{\mathcal{Q}}$ is used in place of Li's $V_{\mathcal{Q}}$ which results in minor but inconsequential variations.

\section{More ON $W_{\mathcal{Q}}$}

In this section we extend the definition of the operator $W_{\mathcal{Q}}(\Phi)$ somewhat by allowing $\Phi$ to be a Hecke character extending $\psi_{\mathcal{Q}}$ or $\psi_{\mathcal{Q}} \psi_{\infty}$, and we develop some properties of $W_{\mathcal{Q}}$ which are crucial to analyzing twists of newforms by characters whose conductors are not prime to the level of the newform.

With the notation as in (2.1) we have:

$$
\mathbf{f} \mid W_{\mathcal{Q}}(\Phi)(x)=\bar{\Phi}(\operatorname{det} x) \overline{\psi_{\mathcal{Q}}}\left(\widetilde{b} \widetilde{t}_{\mathfrak{o}} \bmod \mathcal{Q}\right) \overline{\psi_{\mathcal{M}}}(\widetilde{a} \bmod \mathcal{M})(\mathbf{f} \mid y)(x)
$$

In the case that $\Phi$ extends $\psi_{\mathcal{Q}} \psi_{\infty}, W_{\mathcal{Q}}(\Phi)$ maps $\mathcal{M}_{k}(\mathcal{N}, \Psi)$ to $\mathcal{M}_{k}\left(\mathcal{N}, \Psi \bar{\Phi}^{2}\right)$ and thus $\mathfrak{M}_{k}(\mathcal{N}, \psi, m)$ to $\mathfrak{M}_{k}\left(\mathcal{N}, \psi \bar{\psi}_{\mathcal{Q}}^{2},-m\right)$. Except for the difference in the range of $W_{\mathcal{Q}}(\Phi)$, it is easy to see that the rest of Proposition 2.2 as well as Propostions 2.3 and 2.4 remain valid with this extended definition. Moreover, Proposition 2.7 remains valid in the case that the infinite part of at most one of the two Hecke characters $\Psi_{\mathcal{Q}}$ or $\Psi_{\mathcal{Q}^{\prime}}$ extends $\psi_{\infty}$.

Proposition 4.1. Let $\mathbf{f}, \mathbf{g} \in \mathcal{S}_{k}(\mathcal{N}, \Psi) \subset \mathfrak{S}_{k}(\mathcal{N}, \psi, m)$ and let $\Phi$ be a Hecke character extending $\psi_{\mathcal{Q}}$ or $\psi_{\mathcal{Q}} \psi_{\infty}$. Then $\left\langle\mathbf{f}\left|W_{\mathcal{Q}}(\Phi), \mathbf{g}\right| W_{\mathcal{Q}}(\Phi)\right\rangle=\langle\mathbf{f}, \mathbf{g}\rangle$.

Proof. Given $\mathbf{f}=\left(f_{1}, \ldots, f_{h}\right), \mathbf{g}=\left(g_{1}, \ldots, g_{h}\right) \in \mathcal{S}_{k}(\mathcal{N}, \Psi)$, put $\mathbf{f}^{\prime}=\left(f_{1}^{\prime}, \ldots, f_{h}^{\prime}\right)=$ $\mathbf{f} \mid W_{\mathcal{Q}}(\Phi)$ and $\mathbf{g}^{\prime}=\left(g_{1}^{\prime}, \ldots, g_{h}^{\prime}\right)=\mathbf{g} \mid W_{\mathcal{Q}}(\Phi)$ where $y \in G_{A}, y_{\infty}=1$ and $\operatorname{det}\left(y_{0}\right) \mathcal{O}=Q$ as described in $\S 2$. We shall show that $\left\langle\mathbf{f}\left|W_{\mathcal{Q}}(\Phi), \mathbf{g}\right| W_{\mathcal{Q}}(\Phi)\right\rangle=$ $\sum_{\lambda=1}^{h}\left\langle f_{\lambda}^{\prime}, g_{\lambda}^{\prime}\right\rangle=\sum_{\lambda=1}^{h}\left\langle f_{\lambda}, g_{\lambda}\right\rangle=\langle\mathbf{f}, \mathbf{g}\rangle$ by showing that $\left\langle f_{\lambda}^{\prime}, g_{\lambda}^{\prime}\right\rangle=\left\langle f_{\mu(\lambda)}, g_{\mu(\lambda)}\right\rangle$ for each $\lambda$.

Let $z \in \mathcal{H}^{n}$ and let $w_{\infty} \in G L_{2}(\mathbb{R})^{n}$ such that $w_{\infty}(\mathbf{i})=z$. Let $w_{0}=1$ and $w=w_{0} w_{\infty}$. From $(2.1)$ we have that

$$
\begin{aligned}
f_{\lambda}^{\prime} \mid w_{\infty}(\mathbf{i}) & =\operatorname{det}\left(w_{\infty}\right)^{-i m} \mathbf{f} \mid W_{\mathcal{Q}}(\Phi)\left(x_{\lambda}^{-\iota} w_{\infty}\right) \\
& =\operatorname{det}\left(w_{\infty}\right)^{-i m} \bar{\Phi}\left(\operatorname{det} x_{\lambda}^{-\iota} w_{\infty}\right) \overline{\psi_{\mathcal{Q}}}\left(\widetilde{b} \widetilde{t}_{\mathfrak{o}} \bmod \mathcal{Q}\right) \overline{\psi_{\mathcal{M}}}(\widetilde{a} \bmod \mathcal{M}) \mathbf{f}\left(x_{\lambda}^{-\iota} y^{\iota} w_{\infty}\right) .
\end{aligned}
$$

For each index $\lambda$ there is a uniquely determined index $\mu=\mu(\lambda)$ and a totally positive element $a_{\mu} \in K$ such that $\widetilde{t}_{\lambda}^{-1} \operatorname{det}(y)=a_{\mu} \widetilde{t}_{\mu}^{-1}$. By (1.1) we have that $x_{\lambda}^{-\iota} y^{\iota}=\beta x_{\mu}^{-\iota} v$ with $\beta \in G_{K}$ and $v \in W(\mathcal{N})$. Comparing infinite parts, we see that $v_{\infty}=\beta^{-1}$ hence $\beta \in G L_{2}^{+}(K)$. Thus

$$
\begin{aligned}
f_{\lambda}^{\prime} \mid w_{\infty}(\mathbf{i}) & =\operatorname{det}\left(w_{\infty}\right)^{-i m} \bar{\Phi}\left(\operatorname{det} x_{\lambda}^{-\iota}\right) \overline{\psi_{\mathcal{Q}}}\left(\widetilde{b}_{t_{\mathfrak{d}}} \bmod \mathcal{Q}\right) \overline{\psi_{\mathcal{M}}}(\widetilde{a} \bmod \mathcal{M}) \mathbf{f}\left(\beta x_{\mu}^{-\iota} v w_{\infty}\right) \\
& =\bar{\Phi}\left(\operatorname{det} x_{\lambda}^{-\iota}\right) \overline{\psi_{\mathcal{Q}}}\left(\widetilde{b} \tilde{t}_{\mathfrak{d}} \bmod \mathcal{Q}\right) \overline{\psi_{\mathcal{M}}}(\widetilde{a} \bmod \mathcal{M}) \psi_{Y}\left(v^{\iota}\right) \operatorname{det}\left(v_{\infty}\right)^{i m} f_{\mu} \mid v_{\infty} w_{\infty}(\mathbf{i})
\end{aligned}
$$

from which it follows that

$$
f_{\lambda}^{\prime}(z)=\bar{\Phi}\left(\operatorname{det} x_{\lambda}^{-\iota}\right) \overline{\psi_{\mathcal{Q}}}\left(\widetilde{b} \widetilde{t}_{\mathfrak{d}} \bmod \mathcal{Q}\right) \overline{\psi_{\mathcal{M}}}(\widetilde{a} \bmod \mathcal{M}) \psi_{Y}\left(v^{\iota}\right) \operatorname{det}\left(v_{\infty}\right)^{i m} f_{\mu} \mid \beta^{-1}(z),
$$


$\beta \in G L_{2}^{+}(K)$. The expression for $g_{\lambda}^{\prime}$ is identical, so it is trivial to see that $f_{\lambda}^{\prime}(z) \overline{g_{\lambda}^{\prime}(z)}=f_{\mu} \mid \beta^{-1}(z) \overline{g_{\mu} \mid \beta^{-1}(z)}$, hence $\left\langle f_{\lambda}^{\prime}, g_{\lambda}^{\prime}\right\rangle=\left\langle f_{\mu}\left|\beta^{-1}, g_{\mu}\right| \beta^{-1}\right\rangle=\left\langle f_{\mu}, g_{\mu}\right\rangle$ which completes the proof.

Proposition 2.3 implies that $W_{\mathcal{Q}}(\Phi)$ maps $\mathcal{S}_{k}^{-}(\mathcal{N}, \Psi)$ to $\mathcal{S}_{k}^{-}\left(\mathcal{N}, \Psi \bar{\Phi}^{2}\right)$, and Lemma 4.1 implies that it maps $\mathcal{S}_{k}^{+}(\mathcal{N}, \Psi)$ to $\mathcal{S}_{k}^{+}\left(\mathcal{N}, \Psi \bar{\Phi}^{2}\right)$. By Propostion $2.4, W_{\mathcal{Q}}(\Phi)$ takes Hecke eigenforms to Hecke eigenforms, hence $W_{\mathcal{Q}}(\Phi)$ takes newforms to newforms. Thus if $\mathbf{f} \in \mathcal{S}_{k}(\mathcal{N}, \Psi)$ is a normalized newform, we have

$$
\mathbf{f} \mid W_{\mathcal{Q}}(\Phi)=\lambda_{\mathcal{Q}, \Phi}(\mathbf{f}) \mathbf{g}=\lambda_{\mathcal{Q}}(\mathbf{f}) \mathbf{g}
$$

where $\mathbf{g}$ is a normalized newform in $\mathcal{S}_{k}\left(\mathcal{N}, \Psi \bar{\Phi}^{2}\right)$. Following [1], we call $\lambda_{\mathcal{Q}, \Phi}(\mathbf{f})$ the pseudo-eigenvalue of $W_{\mathcal{Q}}(\Phi)$ at $\mathbf{f}$. It follows from Proposition 2.4 that $\mathbf{f}=\mathbf{g}$ if $\psi_{\mathcal{Q}}=1$, and from Proposition 2.2 it follows that $\lambda_{\mathcal{Q}, \Phi}(\mathbf{f}) \lambda_{\mathcal{Q}, \Phi}(\mathbf{g})=\psi_{\mathcal{Q}}(-1)$, so that in particular, $\lambda_{\mathcal{Q}, \Phi}(\mathbf{f}) \neq 0$. Finally we have defined $W_{\mathcal{Q}}$ so that when $\mathbf{f} \in \mathcal{S}_{k}(\mathcal{N}, \Psi)$ and $\mathcal{Q}=\mathcal{N}$, f $\left|W_{\mathcal{Q}}(\Psi)=\mathbf{f}\right| H_{\mathcal{N}}$; consequently, if $\mathbf{f}$ is a normalized newform in $\mathcal{S}_{k}(\mathcal{N}, \Psi)$, then by Proposition 2.10 of [11] we have $\mathbf{f}\left|H_{\mathcal{N}}=\gamma \mathbf{f}\right| K($ with $|\gamma|=1$ ) hence $\mathbf{f}\left|W_{\mathcal{N}}(\Psi)=\lambda_{\mathcal{N}, \Psi}(\mathbf{f}) \mathbf{f}\right| K$ with $\left|\lambda_{\mathcal{N}, \Psi}\right|=1$. That the pseudo-eigenvalue of $W_{\mathcal{Q}}$ has modulus 1 is true in general:

Theorem 4.2. Let $\mathcal{Q} \mid \mathcal{N}$ with $\left(\mathcal{Q}, \mathcal{N} \mathcal{Q}^{-1}\right)=1$, and let $\Psi_{\mathcal{Q}}$ be a Hecke character extending $\psi_{\mathcal{Q}}$ or $\psi_{\mathcal{Q}} \psi_{\infty}$. Let $\mathbf{f} \in \mathcal{S}_{k}(\mathcal{N}, \Psi)$ be a normalized newform with $\mathbf{f} \mid W_{\mathcal{Q}}\left(\Psi_{\mathcal{Q}}\right)=\lambda_{\mathcal{Q}, \Psi_{\mathcal{Q}}}(\mathbf{f}) \mathbf{g}$ where $\mathbf{g}$ is a normalized newform in $\mathcal{S}_{k}\left(\mathcal{N}, \Psi{\overline{\Psi_{\mathcal{Q}}}}^{2}\right)$. Then $\left|\lambda_{\mathcal{Q}, \Psi_{\mathcal{Q}}}(\mathbf{f})\right|=1$.

To prove this we first require a lemma.

Lemma 4.3. With the notation as above and $\mathfrak{p}$ a prime ideal, the $\mathfrak{p}^{\text {th }}$ Fourier coefficients of $\mathbf{f}$ and $\mathbf{g}$ are related as follows:

$$
C(\mathfrak{p}, \mathbf{g})= \begin{cases}{\overline{\Psi_{\mathcal{Q}}}}^{*}(\mathfrak{p}) C(\mathfrak{p}, \mathbf{f}) & \text { if } \mathfrak{p} \nmid \mathcal{Q} \\ \left(\Psi \Psi_{\mathcal{Q}}^{-1}\right)^{*}(\mathfrak{p}) \overline{C(\mathfrak{p}, \mathbf{f})} & \text { if } \mathfrak{p} \mid \mathcal{Q}\end{cases}
$$

Proof. If $\mathfrak{p} \nmid \mathcal{Q}$ then Proposition 2.4 yields

$$
\mathbf{f}\left|T_{\mathfrak{p}}\right| W_{\mathcal{Q}}\left(\Psi_{\mathcal{Q}}\right)=\Psi_{\mathcal{Q}}^{*}(\mathfrak{p}) \mathbf{f}\left|W_{\mathcal{Q}}\left(\Psi_{\mathcal{Q}}\right)\right| T_{\mathfrak{p}}
$$

from which we obtain

$$
C(\mathfrak{p}, \mathbf{f}) \lambda_{\mathcal{Q}, \Psi_{\mathcal{Q}}}(\mathbf{f}) \mathbf{g}=\Psi_{\mathcal{Q}}^{*}(\mathfrak{p}) \lambda_{\mathcal{Q}, \Psi_{\mathcal{Q}}}(\mathbf{f}) \mathbf{g} \mid T_{\mathfrak{p}}
$$

It follows that

$$
C(\mathfrak{p}, \mathbf{f}) D(s, \mathbf{g})=\Psi_{\mathcal{Q}}^{*}(\mathfrak{p}) D\left(s, \mathbf{g} \mid T_{\mathfrak{p}}\right),
$$

where $D(s, \mathbf{h})$ is the Dirichlet series attached to $\mathbf{h}$. For a fixed integral ideal $\mathfrak{m}$, the coefficients of $N(\mathfrak{m})^{-s}$ in the above Dirichlet series are $C(\mathfrak{p}, \mathbf{f}) C(\mathfrak{m}, \mathbf{g})$ and $\Psi_{\mathcal{Q}}^{*}(\mathfrak{p})\left[C(\mathfrak{m p}, \mathbf{g})+\left(\Psi{\overline{\Psi_{\mathcal{Q}}}}^{2}\right)^{*}(\mathfrak{p}) N(\mathfrak{p})^{k_{0}-1} C\left(\mathfrak{m p} \mathfrak{p}^{-1}, \mathbf{g}\right)\right]$ respectively. When $\mathfrak{m}=\mathcal{O}$, the coefficients must be equal which yields the result in the first case. 
Next assume that $\mathfrak{p} \mid \mathcal{Q}$. By Proposition 2.7 with $\mathcal{Q}^{\prime}=\mathcal{N} \mathcal{Q}^{-1}$, we have

$$
\mathbf{f}\left|W_{\mathcal{N}}(\Psi)=\Psi_{\mathcal{Q}}^{*}\left(\mathcal{N} \mathcal{Q}^{-1}\right) \mathbf{f}\right| W_{\mathcal{Q}}\left(\Psi_{\mathcal{Q}}\right) \mid W_{\mathcal{N} \mathcal{Q}^{-1}}\left(\Psi \Psi_{\mathcal{Q}}^{-1}\right)
$$

which implies

$$
\begin{aligned}
& \lambda_{\mathcal{N}, \Psi}(\mathbf{f}) \mathbf{f}|K| T_{\mathfrak{p}}=\Psi_{\mathcal{Q}}^{*}\left(\mathcal{N} \mathcal{Q}^{-1}\right) \mathbf{f}\left|W_{\mathcal{Q}}\left(\Psi_{\mathcal{Q}}\right)\right| W_{\mathcal{N} \mathcal{Q}^{-1}}\left(\Psi \Psi_{\mathcal{Q}}^{-1}\right) \mid T_{\mathfrak{p}} \\
& =\Psi_{\mathcal{Q}}^{*}\left(\mathcal{N} \mathcal{Q}^{-1}\right)\left(\overline{\Psi \Psi_{\mathcal{Q}}^{-1}}\right)^{*}(\mathfrak{p}) \mathbf{f}\left|W_{\mathcal{Q}}\left(\Psi_{\mathcal{Q}}\right)\right| T_{\mathfrak{p}} \mid W_{\mathcal{N} \mathcal{Q}^{-1}}\left(\Psi \Psi_{\mathcal{Q}}^{-1}\right)
\end{aligned}
$$

and hence

$$
\lambda_{\mathcal{N}, \Psi}(\mathbf{f}) \overline{C(\mathfrak{p}, \mathbf{f})} \mathbf{f}\left|K=\Psi_{\mathcal{Q}}^{*}\left(\mathcal{N} \mathcal{Q}^{-1}\right)\left(\overline{\Psi \Psi_{\mathcal{Q}}^{-1}}\right)^{*}(\mathfrak{p}) \mathbf{f}\right| W_{\mathcal{Q}}\left(\Psi_{\mathcal{Q}}\right)\left|T_{\mathfrak{p}}\right| W_{\mathcal{N} \mathcal{Q}^{-1}}\left(\Psi \Psi_{\mathcal{Q}}^{-1}\right)
$$

On the other hand,

$$
\begin{aligned}
\lambda_{\mathcal{N}, \Psi}(\mathbf{f}) \overline{C(\mathfrak{p}, \mathbf{f})} \mathbf{f} \mid K & =\overline{C(\mathfrak{p}, \mathbf{f})} \mathbf{f} \mid W_{\mathcal{N}}(\Psi) \\
& =\overline{C(\mathfrak{p}, \mathbf{f})} \Psi_{\mathcal{Q}}^{*}\left(\mathcal{N} \mathcal{Q}^{-1}\right) \mathbf{f}\left|W_{\mathcal{Q}}\left(\Psi_{\mathcal{Q}}\right)\right| W_{\mathcal{N} \mathcal{Q}^{-1}}\left(\Psi \Psi_{\mathcal{Q}}^{-1}\right)
\end{aligned}
$$

which implies

$$
\overline{C(\mathfrak{p}, \mathbf{f})} \mathbf{f}\left|W_{\mathcal{Q}}\left(\Psi_{\mathcal{Q}}\right)=\left(\overline{\Psi \Psi_{\mathcal{Q}}^{-1}}\right)^{*}(\mathfrak{p}) \mathbf{f}\right| W_{\mathcal{Q}}\left(\Psi_{\mathcal{Q}}\right) \mid T_{\mathfrak{p}}
$$

or equivalently,

$$
\overline{C(\mathfrak{p}, \mathbf{f})} \mathbf{g}=\left(\overline{\Psi \Psi_{\mathcal{Q}}^{-1}}\right)^{*}(\mathfrak{p}) \mathbf{g} \mid T_{\mathfrak{p}}
$$

For a fixed integral ideal $\mathfrak{m}$, the coefficients of $N(\mathfrak{m})^{-s}$ in the associated Dirichlet series are $\overline{C(\mathfrak{p}, \mathbf{f})} C(\mathfrak{m}, \mathbf{g})$ and $\left(\overline{\Psi \Psi_{\mathcal{Q}}^{-1}}\right)^{*}(\mathfrak{p}) C(\mathfrak{m} \mathfrak{p}, \mathbf{g})$ respectively. When $\mathfrak{m}=\mathcal{O}$, they must be equal, hence $\overline{C(\mathfrak{p}, \mathbf{f})}=\left(\overline{\Psi \Psi_{\mathcal{Q}}^{-1}}\right)^{*}(\mathfrak{p}) C(\mathfrak{p}, \mathbf{g})$ which completes the proof.

Proof (of Theorem 4.2). We first claim that the $\mathfrak{m}^{\text {th }}$ Fourier coefficients of $\mathbf{f}$ and $\mathbf{g}$ have the same modulus. Since $\mathbf{f}$ and $\mathbf{g}$ are both newforms, it suffices to show this (see Theorem 3.3) when $\mathfrak{m}=\mathfrak{p}^{r}, \mathfrak{p}$ a prime. The case of $r=1$ is Lemma 4.3, and the general result follows from equation (1.5) by an easy induction on $r$.

For $\mathbf{f} \in \mathcal{S}_{k}(\mathcal{N}, \psi, m)$ and $\mathbf{g} \in \mathcal{S}_{k}(\mathcal{N}, \phi,-m)$ Shimura defines (p 355 of [12])

$$
L(s ; \mathbf{f}, \mathbf{g})=\sum_{\mathfrak{m} \subset \mathcal{O}} C(\mathfrak{m}, \mathbf{f}) C(\mathfrak{m}, \mathbf{g}) N(\mathfrak{m})^{-s}
$$

and he discusses its analytic continuation. From the proof of Theorem 9.8 of [12] (see also Proposition 4.9 and 4.13 of [11]) we have that the residue of $L(s ; \mathbf{f} \mid K, \mathbf{f})$ at $s=0$ is equal to $\kappa\langle\mathbf{f}, \mathbf{f}\rangle$ where $\kappa$ is a nonzero constant, and $L(s, \mathbf{g} \mid K, \mathbf{g})=$ $\kappa\langle\mathbf{g}, \mathbf{g}\rangle$ for the same constant $\kappa$. However from above we have that $L(s ; \mathbf{f} \mid K, \mathbf{f})=$ $L(s, \mathbf{g} \mid K, \mathbf{g})$ hence $\langle\mathbf{f}, \mathbf{f}\rangle=\langle\mathbf{g}, \mathbf{g}\rangle$. On the other hand, by Proposition 4.1 we have

$$
\langle\mathbf{f}, \mathbf{f}\rangle=\left\langle\mathbf{f}\left|W_{\mathcal{Q}}\left(\Psi_{\mathcal{Q}}\right), \mathbf{f}\right| W_{\mathcal{Q}}\left(\Psi_{\mathcal{Q}}\right)\right\rangle=\left|\lambda_{\mathcal{Q}, \Psi_{\mathcal{Q}}}(\mathbf{f})\right|^{2}\langle\mathbf{g}, \mathbf{g}\rangle=\langle\mathbf{g}, \mathbf{g}\rangle
$$

from which the theorem follows. 


\section{Twists of Newforms}

In this section we characterize the exact level of twists of newforms and give the strong relationship between nonvanishing of eigenvalues of Hecke operators and whether the twist of a newform is again a newform.

Let $\Phi$ be a Hecke character with conductor dividing $\mathcal{M}$. Here and henceforth, the word conductor will mean only the finite part of the conductor. The infinite part of $\Phi$ has the form $\Phi_{\infty}(a)=\operatorname{sgn}(a)^{\ell}|a|^{i r}$ for $\ell \in \mathbb{Z}^{n}, r \in \mathbb{R}^{n}$ and $a \in K_{\infty}^{\times}$; typically in the applications below, we shall choose $r=0$. Let $\tau(\Phi)$ be the Gauss sum defined in (9.31) of [12].

Definition. Let $\mathbf{f} \in \mathcal{S}_{k}(\mathcal{N}, \Psi) \subset \mathfrak{S}_{k}(\mathcal{N}, \psi, m)$. Let

$$
\mathbf{f}\left|R_{\Phi}(\mathcal{M})=\Phi(\operatorname{det} x) \sum_{\nu \in \mathcal{M}^{-1} \mathfrak{d}^{-1} / \mathfrak{d}^{-1}} \bar{\Phi}_{\infty}(\nu) \bar{\Phi}^{*}(\nu \mathcal{M} \mathfrak{d}) \mathbf{f}\right|\left(\begin{array}{ll}
1 & \nu \\
0 & 1
\end{array}\right)_{0}(x) .
$$

The twist of $\mathbf{f}$ by $\Phi, \mathbf{f}_{\Phi}$, is defined by

$$
\mathbf{f}_{\Phi}(x)=\tau(\bar{\Phi})^{-1} \mathbf{f} \mid R_{\Phi}(\operatorname{cond}(\Phi))
$$

Proposition 5.1. Let the notation be as above. If $\mathcal{N}_{0}$ is the conductor of $\Psi, \mathcal{M}_{0}$ is the conductor of $\Phi$, and $\mathcal{L}=\operatorname{lcm}\left(\mathcal{N}, \mathcal{M}_{0} \mathcal{N}_{0}, \mathcal{M}_{0}^{2}\right)$, then $\mathbf{f}_{\Phi} \in \mathcal{S}_{k}\left(\mathcal{L}, \Psi \Phi^{2}\right)$ and $C\left(\mathfrak{m}, \mathbf{f}_{\Phi}\right)=\Phi^{*}(\mathfrak{m}) C(\mathfrak{m}, \mathbf{f})$ for all integral ideals $\mathfrak{m}$.

Proof. This is Proposition 9.7 of [12] (cf. Propositions 4.4 and 4.5 of [11]).

Similarly it is easy to see that $\mathbf{f} \mid R_{\Phi}(\mathcal{M}) \in \mathcal{S}_{k}\left(\mathcal{L}, \Psi \Phi^{2}\right)$ where $\mathcal{L}=\operatorname{lcm}\left(\mathcal{N}, \mathcal{M} \mathcal{N}_{0}, \mathcal{M}^{2}\right)$

The following two Propositions are trivial to verify using the action of the various operators on Fourier coefficients. Note that the Fourier coefficients $C(\mathfrak{m}, \mathbf{f})$ capture the modular form $\mathbf{f}$, although the Dirichlet series $D(s, \mathbf{f})$ need not.

Proposition 5.2. Let $\Phi$ be a Hecke character with conductor $\mathcal{M}$, and let $\mathfrak{p}$ be a prime with $\mathfrak{p} \nmid \mathcal{M}$. Then for $\mathbf{f} \in \mathcal{S}_{k}(\mathcal{N}, \Psi)$ we have $\mathbf{f}_{\Phi} \mid T_{\mathfrak{p}}=\Phi^{*}(\mathfrak{p})\left(\mathbf{f} \mid T_{\mathfrak{p}}\right)_{\Phi}$.

Proposition 5.3. Let $\Phi$ be a Hecke character with conductor $\mathcal{M}$ and $\mathbf{f} \in \mathcal{S}_{k}(\mathcal{N}, \Psi)$. Then $\mathbf{f}_{\Phi} \mid K=(\mathbf{f} \mid K)_{\bar{\Phi}}$.

A simple and straightforward computation gives us

Proposition 5.4. Let $\mathbf{f} \in \mathcal{S}_{k}(\mathcal{N}, \Psi), \mathcal{Q} \mid \mathcal{N}$ with $\left(\mathcal{Q}, \mathcal{N Q}^{-1}\right)=1$. Let $\Phi$ be a Hecke character with conductor $\mathcal{M}$ and suppose that $(\mathcal{Q}, \mathcal{M})=1$. Finally, let $\Psi_{\mathcal{Q}}$ be a Hecke character extending $\psi_{\mathcal{Q}}$. Then $\mathbf{f}_{\Phi} \mid W_{\mathcal{Q}}\left(\Psi_{\mathcal{Q}}\right)=\Phi^{*}(\mathcal{Q})\left(\mathbf{f} \mid W_{\mathcal{Q}}\left(\Psi_{\mathcal{Q}}\right)\right)_{\Phi}$.

Now we wish to explore the actions of newforms under character twists. When the conductor of the character by which we twist is prime to the level of the modular form, the situation is completely straightforwward. We have (cf. comments on p228 of [1])

Theorem 5.5. Let $\mathbf{f} \in \mathcal{S}_{k}(\mathcal{N}, \Psi)$ be a normalized newform and let $\Phi$ be a Hecke character with conductor $\mathcal{M}$. If $(\mathcal{M}, \mathcal{N})=1$ then $\mathbf{f}_{\Phi}$ is a normalized newform in $\mathcal{S}_{k}\left(\mathcal{N M}^{2}, \Psi \Phi^{2}\right)$.

Proof. Since $\mathbf{f}$ is a normalized newform, Theorem 3.3 tells us that $D(s, \mathbf{f})$ has an Euler product representation; consequently, we immediately see that $D\left(s, \mathbf{f}_{\Phi}\right)=$ 
$\sum_{\mathfrak{m} \subset \mathcal{O}} C\left(\mathfrak{m}, \mathbf{f}_{\Phi}\right) N(\mathfrak{m})^{-s}$ has the Euler product

$$
\begin{aligned}
D\left(s, \mathbf{f}_{\Phi}\right)= & \prod_{\mathfrak{q} \mid \mathcal{N}}\left(1-\Phi^{*}(\mathfrak{q}) C(\mathfrak{q}, \mathbf{f}) N(\mathfrak{q})^{-s}\right)^{-1} \\
& \prod_{\mathfrak{p} \nmid \mathcal{N}}\left(1-\Phi^{*}(\mathfrak{p}) C(\mathfrak{p}, \mathbf{f}) N(\mathfrak{p})^{-s}+\Psi^{*}(\mathfrak{p}) \Phi^{*}(\mathfrak{p})^{2} N(\mathfrak{p})^{k_{0}-1-2 s}\right)^{-1} .
\end{aligned}
$$

Moreover, a computation analogous to Proposition 3.65 of [10] (see also equation 9.32 of [12] and Proposition 4.5 of [11] - beware of the two typographical errors) yields that $\mathbf{f}_{\Phi} \mid H_{N M^{2}}=\gamma^{\prime}\left(\mathbf{f} \mid H_{N}\right)_{\bar{\Phi}}$ for $\gamma^{\prime} \in \mathbb{C}^{\times}$with $\left|\gamma^{\prime}\right|=1$. Also recall that since $\mathbf{f}$ is a newform, $\mathbf{f}|K| H_{N}=\gamma \mathbf{f}$ for some $\gamma \in \mathbb{C}^{\times}$with $|\gamma|=1$. Thus

$$
\begin{aligned}
\mathbf{f}_{\Phi}|K| H_{N M^{2}} & =(-1)^{k} \mathbf{f}_{\Phi}\left|H_{N M^{2}}\right| K=(-1)^{k}\left(\gamma^{\prime}\left(\mathbf{f} \mid H_{N}\right)_{\bar{\Phi}}\right) \mid K \\
& =(-1)^{k} \overline{\gamma^{\prime}}\left(\mathbf{f}\left|H_{N}\right| K\right)_{\Phi}=\overline{\gamma^{\prime}}\left(\mathbf{f}|K| H_{N}\right)_{\Phi}=\overline{\gamma^{\prime}} \gamma \mathbf{f}_{\Phi} .
\end{aligned}
$$

The result now follows from Theorem 3.7.

Next we turn to the more complicated case in which we consider a Hecke character whose conductor is not relatively prime to the level. Clearly it suffices to consider characters whose conductor is a prime power which divides the level. We first need a lemma (cf. Lemma 7 of [5]).

Lemma 5.6. Suppose that $\mathbf{f} \in \mathcal{S}_{k}(\mathcal{M}, \Psi) \cap \mathcal{S}_{k}(\mathcal{N}, \Psi)$. Then $\mathbf{f} \in \mathcal{S}_{k}(\mathcal{D}, \Psi)$ where $\mathcal{D}$ is the greatest common divisor of $\mathcal{M}$ and $\mathcal{N}$.

Proof. It is clear that $\Psi$ is defined modulo $\mathcal{D} \mathfrak{P}_{\infty}$. Write $\mathcal{N}=\mathfrak{p}^{\alpha} \mathcal{N}^{\prime}$ and $\mathcal{M}=\mathfrak{p}^{\beta} \mathcal{M}^{\prime}$ where $\mathfrak{p}$ is a prime dividing $\mathcal{M N}$ and with $\mathfrak{p} \nmid \mathcal{M}^{\prime} \mathcal{N}^{\prime}$. Wlog assume $\alpha>\beta$. Then $\mathbf{f} \in \mathcal{S}_{k}(\mathcal{N}, \Psi) \cap \mathcal{S}_{k}\left(\mathfrak{p}^{\alpha-1} \mathcal{M}^{\prime}, \Psi\right)$. By induction, it suffices to show that $\mathbf{f} \in$ $\mathcal{S}_{k}\left(\mathfrak{p}^{\alpha-1} \mathcal{N}^{\prime}, \Psi\right)$.

Since $\mathfrak{p} \nmid \mathcal{N}^{\prime} \mathcal{M}^{\prime}$ we may decompose $W\left(\mathfrak{p}^{\alpha-1} \mathcal{N}^{\prime}\right)$ as $W\left(\mathfrak{p}^{\alpha-1} \mathcal{N}^{\prime}\right)=\cup_{j} W(\mathcal{N}) w_{j}$ where $w_{j} \in W\left(\mathfrak{p}^{\alpha-1} \mathcal{N}^{\prime} \mathcal{M}^{\prime}\right)$. Thus for any $w \in W\left(\mathfrak{p}^{\alpha-1} \mathcal{N}^{\prime}\right)$ with $w_{\infty}=1$ we have $w=w_{1} w_{2}$ where $w_{1} \in W(\mathcal{N}), w_{2} \in W\left(\mathfrak{p}^{\alpha-1} \mathcal{M}^{\prime}\right)$ and $\left(w_{1}\right)_{\infty}=\left(w_{2}\right)_{\infty}=1$. Then $\mathbf{f}|w=\mathbf{f}| w_{1} w_{2}=\psi_{Y}\left(w_{2}\right) \mathbf{f} \mid w_{1}=\psi_{Y}\left(w_{2}\right) \psi_{Y}\left(w_{1}\right) \mathbf{f}=\psi_{Y}(w) \mathbf{f}$, so by equation(1) following (1.3), $\mathbf{f} \in \mathcal{S}_{k}\left(\mathfrak{p}^{\alpha-1} \mathcal{N}^{\prime}\right)$ as desired.

Now we determine the exact level of a twist by a character whose conductor is not prime to the level (cf. Theorem 3.1 of [1]). For an integral ideal $\mathcal{N}$ and a prime $\mathfrak{q}$ dividing $\mathcal{N}$, let $\mathcal{Q}=\mathfrak{q}^{\gamma}$ where $\gamma=\operatorname{ord}_{\mathfrak{q}}(\mathcal{N})$. Then $\mathcal{N}=\mathcal{Q} \mathcal{M}$ with $(\mathcal{Q}, \mathcal{M})=1$; we call $\mathcal{Q}$ the $\mathfrak{q}$-primary part of $\mathcal{N}$.

Theorem 5.7. Let $\mathcal{N}$ be an integral ideal and let $\mathfrak{q}$ be a prime, $\mathfrak{q} \mid \mathcal{N}$. Write $\mathcal{N}=\mathcal{Q} \mathcal{M}$ where $\mathcal{Q}$ is the $\mathfrak{q}$-primary part of $\mathcal{N}$. Take $\mathbf{f}$ to be a normalized newform in $\mathcal{S}_{k}(\mathcal{N}, \Psi)$ with $\operatorname{ord}_{\mathfrak{q}}(\operatorname{cond}(\Psi))=\alpha, \alpha \geq 0$. Let $\Phi$ be a character of conductor $\mathfrak{q}^{\beta}$, $\beta \geq 1$, and put $\mathcal{Q}^{\prime}=\operatorname{lcm}\left(\mathcal{Q}, \mathfrak{q}^{\alpha+\beta}, \mathfrak{q}^{2 \beta}\right)$. Then:

(1) For each prime $\mathfrak{p} \mid \mathcal{M}, \mathbf{f}_{\Phi}$ is not of level $\mathcal{Q}^{\prime} \mathcal{M p}^{-1}$.

(2) The exact level of $\mathbf{f}_{\Phi}$ is $\mathcal{Q}^{\prime} \mathcal{M}$ provided that

(a) $\max (\alpha+\beta, 2 \beta)<\operatorname{ord}_{\mathfrak{q}}(\mathcal{Q})$ if $\mathcal{Q}^{\prime}=\mathcal{Q}$, or

(b) $\operatorname{ord}_{\mathfrak{q}}(\operatorname{cond}(\Psi \Phi))=\max (\alpha, \beta)$ if $\operatorname{ord}_{\mathfrak{q}}\left(\mathcal{Q}^{\prime}\right)>\operatorname{ord}_{\mathfrak{q}}(\mathcal{Q})$. 
Remarks. (1) Theorem 7.1 gives a refinement of this theorem in which essentially, the phrase "the exact level of $\mathbf{f}_{\Phi}$ is $\mathcal{Q}^{\prime} \mathcal{M}$ " can be replaced by the phrase " $\boldsymbol{f}_{\Phi}$ is a newform of level $\mathcal{Q}^{\prime} \mathcal{M}$ ". (2) There are cases in which the level of $\mathbf{f}_{\Phi}$ is a proper divisor of $\mathcal{Q}^{\prime} \mathcal{M}$ (see Theorem $7.2)$.

Proof. By Proposition 5.1, $\mathbf{f}_{\Phi} \in \mathcal{S}_{k}\left(\mathcal{Q}^{\prime} \mathcal{M}, \Psi \Phi^{2}\right)$. If $\mathbf{f}_{\Phi} \in \mathcal{S}_{k}\left(\mathcal{Q}^{\prime} \mathcal{M} \mathfrak{p}^{-1}, \Psi \Phi^{2}\right)$, then $\left(\mathbf{f}_{\Phi}\right)_{\bar{\Phi}} \in \mathcal{S}_{k}(\widetilde{\mathcal{N}}, \Psi)$ where $\widetilde{\mathcal{N}}=\mathfrak{q}^{\gamma} \mathcal{M} \mathfrak{p}^{-1}$, where $\gamma=\max \left(\operatorname{ord}_{\mathfrak{q}}\left(\mathcal{Q}^{\prime}\right), \operatorname{ord}_{\mathfrak{q}}\left(\operatorname{cond}\left(\Psi \Phi^{2}\right)\right)+\right.$ $\beta, 2 \beta)$. Now $\left(\mathbf{f}_{\Phi}\right)_{\bar{\Phi}}$ is equivalent to a newform $\mathbf{h}$, written $\left(\mathbf{f}_{\Phi}\right)_{\bar{\Phi}} \sim \mathbf{h}$ (i.e. has the same eigenvalues for almost all Hecke operators $T_{\mathfrak{l}}, \mathfrak{l}$ a prime) where the level of $\mathbf{h}$ divides $\widetilde{\mathcal{N}}$. By Proposition 5.2, $\mathbf{f} \sim\left(\mathbf{f}_{\Phi}\right)_{\bar{\Phi}}$, so $\mathbf{f} \sim \mathbf{h}$, and so by Theorem 3.5, $\mathbf{f} \in \mathcal{S}_{k}(\widetilde{\mathcal{N}}, \Psi) \cap S_{k}(\mathcal{Q M}, \Psi)$. By Lemma 5.6, $\mathbf{f} \in \mathcal{S}_{k}\left(\mathcal{Q M} \mathfrak{p}^{-1}, \Psi\right)$, a contradiction.

Now suppose that $\mathbf{f}_{\Phi}$ has level $\mathcal{Q}^{\prime} \mathcal{M} \mathfrak{q}^{-1}$. First note that $\mathbf{f}_{\Phi} \neq 0$ otherwise $\mathbf{f} \sim\left(\mathbf{f}_{\Phi}\right)_{\bar{\Phi}}=0$, contradicting that $\mathbf{f}$ is a newform (see Theorem 3.1). Let $w_{j} \in$ $W\left(\mathcal{Q}^{\prime} \mathcal{M} \mathfrak{q}^{-1}\right)\left(\right.$ with $\left.\left(w_{j}\right)_{\infty}=1\right)$ be a set of representatives for $W\left(\mathcal{Q}^{\prime} \mathcal{M}\right) \backslash W\left(\mathcal{Q}^{\prime} \mathcal{M} \mathfrak{q}^{-1}\right)$. Then

$$
\mathbf{f}_{\Phi}\left|\operatorname{Tr}_{\mathcal{Q}^{\prime} \mathcal{M} \mathfrak{q}^{-1}}^{\mathcal{Q}^{\prime} \mathcal{M}}=\sum_{j} \overline{\psi_{Y}}\left(w_{j}\right) \mathbf{f}_{\Phi}\right| w_{j}=\sum_{j} \overline{\psi_{Y}}\left(w_{j}\right) \psi_{Y}\left(w_{j}\right) \mathbf{f}_{\Phi}=N(\mathfrak{q}) \mathbf{f}_{\Phi} .
$$

On the other hand, since we may take $\left\{w_{j}\right\}=\left\{\left(\begin{array}{ll}1 & 0 \\ c & 1\end{array}\right)_{0} \mid c \in \mathcal{Q}^{\prime} \mathcal{M} \mathfrak{q}^{-1} \mathfrak{d} / \mathcal{Q}^{\prime} \mathcal{M} \mathfrak{d}\right\}$,

$$
\begin{aligned}
\mathbf{f}_{\Phi} \mid & \operatorname{Tr}_{\mathcal{Q}^{\prime} \mathcal{M} \mathfrak{q}^{-1}}^{\mathcal{Q}^{\prime} \mathcal{M}}(x)=\sum_{j} \overline{\psi_{Y}}\left(w_{j}\right) \mathbf{f}_{\Phi}\left(x w_{j}^{\iota}\right) \\
& =\tau(\bar{\Phi})^{-1} \Phi(\operatorname{det}(x)) \sum_{\substack{c \in \mathcal{Q}^{\prime} \mathcal{M} \mathfrak{q}^{-1} \mathfrak{d} / \mathcal{Q}^{\prime} \mathcal{M} \mathfrak{d} \\
\nu \in \mathfrak{q}^{-\beta} \mathfrak{d}^{-1} / \mathfrak{d}^{-1}}} \bar{\Phi}_{\infty}(\nu) \bar{\Phi}^{*}\left(\nu \mathfrak{q}^{\beta} \mathfrak{d}\right) \mathbf{f}\left(x\left(\begin{array}{cc}
1 & 0 \\
-c & 1
\end{array}\right)_{0}\left(\begin{array}{cc}
1 & -\nu \\
0 & 1
\end{array}\right)_{0}\right) .
\end{aligned}
$$

Case I: $\mathfrak{q}^{\beta+2} \mid \mathcal{Q}^{\prime}$

The proof is very similar to the proof in [1], but for completeness, we sketch it. Since $\mathfrak{q}^{\beta+2} \mid \mathcal{Q}^{\prime}$, for each $\nu \in \mathfrak{q}^{-\beta} \mathfrak{d}^{-1}$ there is a unique $\nu^{\prime} \in \mathfrak{q}^{-\beta} \mathfrak{d}^{-1}$ such that $\nu-\nu^{\prime}(1+\nu c) \in \mathfrak{d}^{-1}$. It follows that

$$
\mathbf{f}\left(x\left(\begin{array}{cc}
1 & 0 \\
-c & 1
\end{array}\right)_{0}\left(\begin{array}{cc}
1 & -\nu \\
0 & 1
\end{array}\right)_{0}\right)=\mathbf{f}\left(x\left(\begin{array}{cc}
1 & -\nu^{\prime} \\
0 & 1
\end{array}\right)_{0}\left(\begin{array}{cc}
1 & 0 \\
-c & 1
\end{array}\right)_{0} w^{\iota}\right)
$$

where

$$
w_{0}=\left(\begin{array}{cc}
1+c \nu^{\prime}(c \nu+1) & * \\
* & 1-c \nu^{\prime}
\end{array}\right)_{0} \in W\left(\mathcal{Q}^{\prime} \mathcal{M}\right), w_{\infty}=1 .
$$

Equation (5.2) now yields

$$
\begin{aligned}
\mathbf{f}_{\Phi} \mid \operatorname{Tr}_{\mathcal{Q}^{\prime} \mathcal{M} \mathfrak{q}^{-1}}^{\mathcal{Q}^{\prime} \mathcal{M}}(x)= & \sum_{\substack{c \in \mathcal{Q}^{\prime} \mathcal{M} \mathfrak{q}^{-1} \mathfrak{d} / \mathcal{Q}^{\prime} \mathcal{M} \mathfrak{d} \\
\nu \in \mathfrak{q}^{-\beta} \mathfrak{d}-1 / \mathfrak{d}^{-1}}} \bar{\Phi}_{\infty}(\nu) \bar{\Phi}^{*}\left(\nu \mathfrak{q}^{\beta} \mathfrak{d}\right) \mathbf{f}\left(x\left(\begin{array}{cc}
1 & -\nu^{\prime} \\
0 & 1
\end{array}\right)_{0}\left(\begin{array}{cc}
1 & 0 \\
-c & 1
\end{array}\right)_{0} w^{\iota}\right) \\
&
\end{aligned}
$$

Since $W(\mathcal{Q M}) \subset W\left(\mathcal{Q}^{\prime} \mathcal{M}\right)$ we have

$$
\mathbf{f}\left(x\left(\begin{array}{cc}
1 & -\nu^{\prime} \\
0 & 1
\end{array}\right)_{0}\left(\begin{array}{cc}
1 & 0 \\
-c & 1
\end{array}\right)_{0} w^{\iota}\right)=\psi\left(1+c \nu^{\prime}\right) \mathbf{f}\left(x\left(\begin{array}{cc}
1 & -\nu^{\prime} \\
0 & 1
\end{array}\right)_{0}\left(\begin{array}{cc}
1 & 0 \\
-c & 1
\end{array}\right)_{0}\right) .
$$


It is easy to see that $\bar{\Phi}_{\infty}(\nu) \bar{\Phi}^{*}\left(\nu \mathfrak{q}^{\beta} \mathfrak{d}\right)$ depends only on the value of $\nu \bmod \mathfrak{d}^{-1}$ so

$$
\begin{aligned}
\bar{\Phi}_{\infty}(\nu) & \bar{\Phi}^{*}\left(\nu \mathfrak{q}^{\beta} \mathfrak{d}\right) \\
& =\bar{\Phi}_{\infty}\left(\nu^{\prime}(1+\nu c)\right) \bar{\Phi}^{*}\left(\nu^{\prime}(1+\nu c) \mathfrak{q}^{\beta} \mathfrak{d}\right) \\
& =\bar{\Phi}_{\infty}\left(\nu^{\prime}(1+\nu c)\left(1-\nu^{\prime} c\right)\right) \bar{\Phi}^{*}\left(\nu^{\prime}(1+\nu c)\left(1-\nu^{\prime} c\right) \mathfrak{q}^{\beta} \mathfrak{d}\right) \Phi_{\infty}\left(\left(1-\nu^{\prime} c\right)\right) \Phi^{*}\left(\left(1-\nu^{\prime} c\right)\right) \\
& =\bar{\Phi}_{\infty}\left(\nu^{\prime}\right) \bar{\Phi}^{*}\left(\nu^{\prime} \mathfrak{q}^{\beta} \mathfrak{d}\right) \bar{\Phi}_{\mathfrak{q}}\left(1-\nu^{\prime} c\right) \\
& =\bar{\Phi}_{\infty}\left(\nu^{\prime}\right) \bar{\Phi}^{*}\left(\nu^{\prime} \mathfrak{q}^{\beta} \mathfrak{d}\right) \Phi_{\mathfrak{q}}\left(1+\nu^{\prime} c\right)
\end{aligned}
$$

where $\Phi_{\mathfrak{q}}$ is the $\mathfrak{q}$-part of the Hecke character $\Phi$, which may be viewed as a numerical character modulo $\mathfrak{q}^{\beta}$. Thus (5.3) becomes

$$
\begin{aligned}
\mathbf{f}_{\Phi} & \operatorname{Tr}_{\substack{c \in \mathcal{Q}^{\prime} \mathcal{M} \mathfrak{q}^{-1} \mathfrak{d} / \mathcal{Q}^{\prime} \mathcal{M} \mathfrak{d} \\
\nu^{\prime} \in \mathfrak{q}^{-\beta} \mathfrak{d}^{-1} / \mathfrak{d}^{-1}}}^{\mathcal{Q}^{\prime} \mathcal{M}}(x)=\tau(\bar{\Phi})^{-1} \Phi(\operatorname{det}(x)) \times \\
& \Phi_{\infty}\left(\nu^{\prime}\right) \bar{\Phi}^{*}\left(\nu^{\prime} \mathfrak{q}^{\beta} \mathfrak{d}\right) \psi \Phi_{\mathfrak{q}}\left(1+\nu^{\prime} c\right) \mathbf{f}\left(x\left(\begin{array}{cc}
1 & -\nu^{\prime} \\
0 & 1
\end{array}\right)_{0}\left(\begin{array}{cc}
1 & 0 \\
-c & 1
\end{array}\right)_{0}\right) \\
&
\end{aligned}
$$

Subcase A. $\alpha>\beta$, or $\alpha=\beta>(1 / 2) \operatorname{ord}_{\mathfrak{q}} \mathcal{Q}$ and $\operatorname{ord}_{\mathfrak{q}}\left(\operatorname{cond}\left(\psi \Phi_{q}\right)\right)=\alpha$.

If $\operatorname{ord}_{\mathfrak{q}} \mathcal{Q}^{\prime}>\operatorname{ord}_{q} \mathcal{Q}$, then $\operatorname{cond}\left(\psi_{\mathfrak{q}} \Phi_{\mathfrak{q}}\right)=\mathfrak{q}^{\alpha}, c \in \mathcal{N} \mathfrak{d}$, and $c \nu^{\prime} \in \mathfrak{q}^{\alpha-1} \mathcal{M}$. If $\mathcal{Q}^{\prime}=\mathcal{Q}$, then $\max (\alpha+\beta, 2 \beta)<\operatorname{ord}_{\mathfrak{q}} \mathcal{Q}, \operatorname{cond}\left(\psi_{\mathfrak{q}} \Phi_{\mathfrak{q}}\right)=\mathfrak{q}^{\alpha}, c \in \mathcal{Q}^{\prime} \mathcal{M q}^{-1} \mathfrak{d}$, and $c \nu^{\prime} \in \mathfrak{q}^{\alpha} \mathcal{M}$.

Thus equation (5.4) yields

$$
\begin{aligned}
& \tau(\bar{\Phi}) \bar{\Phi}(\operatorname{det}(x)) \mathbf{f}_{\Phi} \mid \operatorname{Tr}_{\mathcal{Q}^{\prime} \mathcal{M} \mathfrak{q}^{-1}}^{\mathcal{Q}^{\prime} \mathcal{M}}(x) \\
& = \begin{cases}\sum_{c} \sum_{\nu^{\prime}} \bar{\Phi}_{\infty}\left(\nu^{\prime}\right) \bar{\Phi}^{*}\left(\nu^{\prime} \mathfrak{q}^{\beta} \mathfrak{d}\right) \psi_{\mathfrak{q}} \Phi_{\mathfrak{q}}\left(1+\nu^{\prime} c\right) \mathbf{f}\left(x\left(\begin{array}{cc}
1 & -\nu^{\prime} \\
0 & 1
\end{array}\right)_{0}\right) & \text { if } \operatorname{ord}_{\mathfrak{q}} \mathcal{Q}^{\prime}>\operatorname{ord}_{q} \mathcal{Q} \\
\sum_{c} \sum_{\nu^{\prime}} \bar{\Phi}_{\infty}\left(\nu^{\prime}\right) \bar{\Phi}^{*}\left(\nu^{\prime} \mathfrak{q}^{\beta} \mathfrak{d}\right) \mathbf{f}\left(x\left(\begin{array}{cc}
1 & -\nu^{\prime} \\
0 & 1
\end{array}\right)_{0}\left(\begin{array}{cc}
1 & 0 \\
-c & 1
\end{array}\right)_{0}\right) & \text { if } \mathcal{Q}^{\prime}=\mathcal{Q}\end{cases} \\
& = \begin{cases}\sum_{\nu^{\prime}} \bar{\Phi}_{\infty}\left(\nu^{\prime}\right) \bar{\Phi}^{*}\left(\nu^{\prime} \mathfrak{q}^{\beta} \mathfrak{d}\right) \mathbf{f}\left(x\left(\begin{array}{cc}
1 & -\nu^{\prime} \\
0 & 1
\end{array}\right)_{0}\right) \sum_{c} \psi_{\mathfrak{q}} \Phi_{\mathfrak{q}}\left(1+\nu^{\prime} c\right) & \text { if } \operatorname{ord}_{\mathfrak{q}} \mathcal{Q}^{\prime}>\operatorname{ord}_{q} \mathcal{Q} \\
\left(\mathbf{f} \mid \operatorname{Tr}_{\mathcal{Q}^{\prime} \mathcal{M} \mathfrak{q}^{-1}}\right)_{\Phi}(x) & \text { if } \mathcal{Q}^{\prime}=\mathcal{Q}\end{cases} \\
& =0
\end{aligned}
$$

in the first case since either $\bar{\Phi}^{*}\left(\nu^{\prime} \mathfrak{q}^{\beta} \mathfrak{d}\right)=0$ or $\sum_{c} \psi_{\mathfrak{q}} \Phi_{\mathfrak{q}}\left(1+\nu^{\prime} c\right)=0$ (for $1-\nu^{\prime} c$ runs over a group on which $\psi_{\mathfrak{q}} \Phi_{\mathfrak{q}}$ is nontrivial), and in the second case by Theorem 3.4. This contradicts equation (5.1).

Subcase B. $\alpha<\beta$ or $\alpha=\beta<(1 / 2) \operatorname{ord}_{\mathfrak{q}} \mathcal{Q}$.

If $\operatorname{ord}_{\mathfrak{q}} \mathcal{Q}^{\prime}>\operatorname{ord}_{q} \mathcal{Q}$, then $\operatorname{cond}\left(\psi_{\mathfrak{q}} \Phi_{\mathfrak{q}}\right)=\mathfrak{q}^{\beta}, c \in \mathcal{N} \mathfrak{d}$, and $c \nu^{\prime} \in \mathfrak{q}^{\beta-1} \mathcal{M}$. If $\mathcal{Q}^{\prime}=\mathcal{Q}$, then $\max (\alpha+\beta, 2 \beta)<\operatorname{ord}_{\mathfrak{q}} \mathcal{Q}, \operatorname{cond}\left(\psi_{\mathfrak{q}} \Phi_{\mathfrak{q}}\right) \mid \mathfrak{q}^{\alpha}, c \in \mathcal{Q}^{\prime} \mathcal{M q}^{-1} \mathfrak{d}$, and $c \nu^{\prime} \in \mathfrak{q}^{\beta} \mathcal{M}$. The argument proceeds in analogy to the one above.

Case II: $\mathfrak{q}^{\beta+2} \nmid \mathcal{Q}^{\prime}$

Here we must have $\mathcal{Q}=\mathfrak{q}, \mathcal{Q}^{\prime}=\mathfrak{q}^{2}$, and $\operatorname{cond}\left(\psi_{\mathfrak{q}} \Phi_{\mathfrak{q}}\right)=\mathfrak{q}=\operatorname{cond}\left(\Phi_{\mathfrak{q}}\right)$. The argument here is in analogy to that in [6]. 
Starting from equation (5.2), it is sufficient to show that

$$
\sum_{\substack{\nu \in \mathfrak{q}^{-1} \mathfrak{d}^{-1} / \mathfrak{d}^{-1} \\
c \in \mathfrak{q} \mathcal{M} \mathfrak{d} / \mathfrak{q}^{2} \mathcal{M} \mathfrak{d}}} \bar{\Phi}_{\infty}(\nu) \bar{\Phi}^{*}(\nu \mathfrak{q} \mathfrak{d}) \mathbf{f}\left|\left(\begin{array}{ll}
1 & \nu \\
0 & 1
\end{array}\right)_{0}\right|\left(\begin{array}{ll}
1 & 0 \\
c & 1
\end{array}\right)_{0}(x)=0
$$

Note that we may assume that $(\nu \mathfrak{q} \mathfrak{d}, \mathfrak{q})=1$ otherwise $\bar{\Phi}^{*}(\nu \mathfrak{q} \mathfrak{d})=0$. We first show that $\sum_{\substack{\nu, c \\ 1+c \nu \in \mathfrak{q}}} \bar{\Phi}_{\infty}(\nu) \bar{\Phi}^{*}(\nu \mathfrak{q} \mathfrak{d}) \mathbf{f}\left|\left(\begin{array}{ll}1 & \nu \\ 0 & 1\end{array}\right)_{0}\right|\left(\begin{array}{ll}1 & 0 \\ c & 1\end{array}\right)_{0}=0$, so given $c, \nu$ assume that $1+c \nu \in \mathfrak{q}$. Let $\widetilde{q} \in K_{A}^{\times}$with $\widetilde{q} \mathcal{O}=\mathfrak{q}$ and $\widetilde{q}_{\infty}=1$, and let $y \in G_{A}$, with $y_{0}=\left(\begin{array}{ll}1 & \nu \\ 0 & 1\end{array}\right)_{0}\left(\begin{array}{ll}1 & 0 \\ c & 1\end{array}\right)_{0}\left(\begin{array}{cc}1 & 0 \\ 0 & \widetilde{q}^{-1}\end{array}\right)_{0}=\left(\begin{array}{cc}1+c \nu & \nu \widetilde{q} \\ c & \widetilde{q}\end{array}\right)_{0}, y_{\infty}=1$. Finally, let $\Psi_{\mathcal{Q}}$ be a Hecke character extending $\psi_{\mathfrak{q}}$. Then we can use $y$ to define the action of $W_{\mathcal{Q}}\left(\Psi_{\mathcal{Q}}\right)$. It follows that

$$
\begin{aligned}
& \sum_{\nu \in \mathfrak{q}^{-1} \mathfrak{d}^{-1} / \mathfrak{d}^{-1}} \sum_{\substack{c \in \mathfrak{q} \mathcal{M} \mathfrak{d} / \mathfrak{q}^{2} \mathcal{M} \mathfrak{d} \\
1+c \nu \in \mathfrak{q}}} \bar{\Phi}_{\infty}(\nu) \bar{\Phi}^{*}(\nu \mathfrak{q} \mathfrak{d}) \mathbf{f}\left|\left(\begin{array}{ll}
1 & \\
0 & 1
\end{array}\right)_{0}\right|\left(\begin{array}{ll}
1 & 0 \\
c & 1
\end{array}\right)_{0}(x) \\
& =\Psi_{\mathcal{Q}}(\widetilde{q} \operatorname{det} x) \sum_{\nu \in \mathfrak{q}^{-1} \mathfrak{d}^{-1} / \mathfrak{d}-1} \sum_{\substack{c \in \mathfrak{q} \mathcal{M} \mathfrak{d} / \mathfrak{q}^{2} \mathcal{M} \mathfrak{d} \\
1+c \nu \in \mathfrak{q}}} \bar{\Phi}_{\infty}(\nu) \bar{\Phi}^{*}(\nu \mathfrak{q} \mathfrak{d}) \psi_{\mathfrak{q}}\left(\nu \widetilde{q} \tilde{t}_{\mathfrak{d}}\right) \mathbf{f}\left|W_{\mathcal{Q}}\left(\Psi_{\mathcal{Q}}\right)\right|\left(\begin{array}{ll}
1 & 0 \\
0 & \widetilde{q}^{-1}
\end{array}\right)_{0}(x) \\
& =\Psi_{\mathcal{Q}}(\widetilde{q} \operatorname{det} x) \bar{\Phi}\left(\widetilde{q} \tilde{t}_{\mathfrak{d}} \nu\right) \sum_{\nu \in \mathfrak{q}^{-1} \mathfrak{d}^{-1} / \mathfrak{d}^{-1}} \Phi_{\mathfrak{q}} \psi_{\mathfrak{q}}\left(\nu \widetilde{q} \widetilde{t}_{\mathfrak{d}}\right) \sum_{\substack{c \in \mathfrak{q} \mathcal{M} \mathfrak{d} / \mathfrak{q}^{2} \mathcal{M} \mathfrak{d} \\
1+c \nu \in \mathfrak{q}}} \mathbf{f}\left|W_{\mathcal{Q}}\left(\Psi_{\mathcal{Q}}\right)\right|\left(\begin{array}{ll}
1 & 0 \\
0 & \widetilde{q}^{-1}
\end{array}\right)_{0}(x) .
\end{aligned}
$$

As $\nu$ runs over $\mathfrak{q}^{-1} \mathfrak{d}^{-1} / \mathfrak{d}^{-1}, \nu \widetilde{q} \widetilde{t_{\mathfrak{d}}}$ runs over $\mathcal{O} / \mathfrak{q}$, and since $\Phi_{\mathfrak{q}} \psi_{\mathfrak{q}} \neq 1$,

$$
\sum_{\nu \in \mathfrak{q}^{-1} \mathfrak{d}^{-1} / \mathfrak{d}^{-1}} \Phi_{\mathfrak{q}} \psi_{\mathfrak{q}}\left(\nu \widetilde{q} \tilde{t}_{\mathfrak{d}}\right)=0
$$

which proves the first claim.

Thus, we are reduced to showing that

$$
\sum_{\nu \in \mathfrak{q}^{-1} \mathfrak{d}^{-1} / \mathfrak{d}^{-1}} \sum_{\substack{c \in \mathfrak{q} \mathcal{M} \mathfrak{d} / \mathfrak{q}^{2} \mathcal{M} \mathfrak{d} \\
1+c \nu \notin \mathfrak{q}}} \bar{\Phi}_{\infty}(\nu) \bar{\Phi}^{*}(\nu \mathfrak{q} \mathfrak{d}) \mathbf{f}\left|\left(\begin{array}{ll}
1 & \nu \\
0 & 1
\end{array}\right)_{0}\right|\left(\begin{array}{ll}
1 & 0 \\
c & 1
\end{array}\right)_{0}(x)=0
$$

For each $c, \nu$ with $1+c \nu \notin \mathfrak{q}$ there is a unique $\nu^{\prime} \in q^{-1} \mathfrak{d}^{-1} / \mathfrak{d}^{-1}$ such that $\nu^{\prime}(1+$ $c \nu) \equiv \nu \bmod \mathfrak{d}^{-1}$. Then $\left(\begin{array}{ll}1 & \nu \\ 0 & 1\end{array}\right)_{0}\left(\begin{array}{ll}1 & 0 \\ c & 1\end{array}\right)_{0}\left(\begin{array}{cc}1 & -\nu^{\prime} \\ 0 & 1\end{array}\right)_{0}=\left(\begin{array}{cc}1+c \nu & \nu-\nu^{\prime}(1+c \nu) \\ c & 1-c \nu^{\prime}\end{array}\right)_{0} \in W(\mathfrak{q} \mathcal{M})=$ $W(\mathcal{N})$. Hence

$$
\begin{aligned}
& \sum_{\substack { \nu \in \mathfrak{q}^{-1} \mathfrak{d}^{-1} / \mathfrak{d}^{-1} \\
\begin{subarray}{c}{c \in \mathfrak{q} \mathcal{M} \mathfrak{d} / \mathfrak{q}^{2} \mathcal{M} \mathfrak{d} \\
1+c \nu \notin \mathfrak{q}{ \nu \in \mathfrak { q } ^ { - 1 } \mathfrak { d } ^ { - 1 } / \mathfrak { d } ^ { - 1 } \\
\begin{subarray} { c } { c \in \mathfrak { q } \mathcal { M } \mathfrak { d } / \mathfrak { q } ^ { 2 } \mathcal { M } \mathfrak { d } \\
1 + c \nu \notin \mathfrak { q } } }\end{subarray}} \bar{\Phi}_{\infty}(\nu) \bar{\Phi}^{*}(\nu \mathfrak{q} \mathfrak{d}) \mathbf{f}\left|\left(\begin{array}{ll}
1 & \nu \\
0 & 1
\end{array}\right)_{0}\right|\left(\begin{array}{ll}
1 & 0 \\
c & 1
\end{array}\right)_{0}(x) \\
= & \sum_{\substack{\nu, c \\
1+c \nu \notin \mathfrak{q}}} \bar{\Phi}_{\infty}\left(\nu^{\prime}(1+c \nu)\right) \bar{\Phi}^{*}\left(\nu^{\prime}(1+c \nu) \mathfrak{q} \mathfrak{d}\right) \psi(1+c \nu) \mathbf{f} \mid\left(\begin{array}{ll}
1 & \nu^{\prime} \\
0 & 1
\end{array}\right)_{0}(x) \\
= & \sum_{\substack{\nu, c \\
1+c \nu \notin \mathfrak{q}}} \bar{\Phi}_{\infty}\left(\nu^{\prime}\right) \bar{\Phi}^{*}\left(\nu^{\prime} \mathfrak{q} \mathfrak{d}\right) \Phi_{\mathfrak{q}} \psi_{\mathfrak{q}}(1+c \nu) \mathbf{f} \mid\left(\begin{array}{ll}
1 & \nu^{\prime} \\
0 & 1
\end{array}\right)_{0}(x) .
\end{aligned}
$$


Observe that $\nu^{\prime}(1+c \nu) \equiv \nu \bmod \mathfrak{d}^{-1}$ iff $\nu^{\prime} \equiv\left(1-c \nu^{\prime}\right) \nu \bmod \mathfrak{d}^{-1}$, so we can change the variables $c, \nu$ to $c, \nu^{\prime}$ where $\nu^{\prime}$ runs over $\mathfrak{q}^{-1} \mathfrak{d}^{-1} / \mathfrak{d}^{-1}$ with $\left(\nu^{\prime} \mathfrak{q} \mathfrak{d}, \mathfrak{q}\right)=1$ and $c$ runs over $\mathfrak{q} \mathcal{M} \mathfrak{d} / \mathfrak{q}^{2} \mathcal{M} \mathfrak{d}$ with $1-c \nu^{\prime} \notin \mathfrak{q}$. Also note that $\Phi_{\mathfrak{q}} \psi_{\mathfrak{q}}(1+c \nu)=\overline{\Phi_{\mathfrak{q}}} \psi_{\mathfrak{q}}\left(1-c \nu^{\prime}\right)$ so equation (5.7) becomes

$$
\sum_{\nu^{\prime} \in \mathfrak{q}^{-1} \mathfrak{d}^{-1} / \mathfrak{d}^{-1}} \bar{\Phi}_{\infty}\left(\nu^{\prime}\right) \bar{\Phi}^{*}\left(\nu^{\prime} \mathfrak{q} \mathfrak{d}\right) \mathbf{f} \mid\left(\begin{array}{ll}
1 & \nu^{\prime} \\
0 & 1
\end{array}\right)_{0}(x) \sum_{\substack{c \in \mathfrak{q} \mathcal{M} \mathfrak{d} / \mathfrak{q}^{2} \mathcal{M} \mathfrak{d} \\
1-c \nu^{\prime} \notin \mathfrak{q}}} \overline{\Phi_{\mathfrak{q}} \psi_{\mathfrak{q}}}\left(1-c \nu^{\prime}\right)=0
$$

since for fixed $\nu^{\prime}, 1-c \nu^{\prime}$ runs over $(\mathcal{O} / \mathfrak{q})^{\times}$, and $\Phi_{\mathfrak{q}} \psi_{\mathfrak{q}}$ is a nontrivial character on $(\mathcal{O} / \mathfrak{q})^{\times}$. This provides the desired contradiction and completes the proof of the theorem.

Now we have

Theorem 5.8. Let $\mathfrak{q} \mid \mathcal{N}$ and let $\mathcal{Q}$ be the $\mathfrak{q}$-primary part of $\mathcal{N}$. Write $\mathcal{N}=\mathcal{Q M}$ and let $\Phi$ be a Hecke character of conductor $\mathfrak{q}^{\beta}, \beta \geq 1$. Let $\mathbf{f}$ be a normalized newform in $\mathcal{S}_{k}(\mathcal{N}, \Psi)$. Then there exists a normalized newform $\mathbf{g} \in \mathcal{S}_{k}\left(\widetilde{\mathcal{Q}} \mathcal{M}, \Psi \Phi^{2}\right)$ with $\widetilde{\mathcal{Q}}=\mathfrak{q}^{\gamma}$ for some $\gamma \geq 0$ such that $\mathbf{f}_{\Phi}=\mathbf{g}-\mathbf{g}\left|T_{\mathfrak{q}}\right| B_{\mathfrak{q}}$.

Remark. In the case $K=\mathbb{Q}$, more precise information can be obtained about $\widetilde{\mathcal{Q}}$ (see Theorem 3.16 of $[3]$ ).

Proof. By Theorem 5.7, $\mathbf{f}_{\Phi} \in \mathcal{S}_{k}\left(\mathcal{Q}^{\prime} \mathcal{M}, \Psi \Phi^{2}\right)$ where $\mathcal{Q}^{\prime}=\operatorname{lcm}\left(\mathcal{Q}, \mathfrak{q}^{\alpha+\beta}, \mathfrak{q}^{2 \beta}\right)$ where $\alpha=\operatorname{ord}_{\mathfrak{q}}(\operatorname{cond}(\Psi))$. Since $\mathbf{f}_{\Phi}$ is a simultaneous eigenform for all $T_{\mathfrak{p}}$ with $\mathfrak{p} \nmid \mathcal{N}$, there exists a newform $\mathbf{g} \in \mathcal{S}_{k}\left(\widetilde{\mathcal{Q}} \mathcal{M}^{\prime}, \Psi \Phi^{2}\right)$ with $\mathbf{f}_{\Phi} \sim$ g. Here $\widetilde{\mathcal{Q}} \mid \mathcal{Q}^{\prime}$ and $\mathcal{M}^{\prime} \mid$ $\mathcal{M}$. Then $\mathbf{g}_{\bar{\Phi}} \sim\left(\mathbf{f}_{\Phi}\right)_{\bar{\Phi}} \sim \mathbf{f}$ and $\mathbf{g}_{\bar{\Phi}}$ has level $\widetilde{\mathcal{Q}}^{\prime} \mathcal{M}^{\prime}$ where $\widetilde{\mathcal{Q}}^{\prime}=\mathfrak{q}^{\gamma}$, where $\gamma=$ $\max \left(\operatorname{ord}_{\mathfrak{q}}(\widetilde{\mathcal{Q}}), 2 \beta, \operatorname{ord}_{\mathfrak{q}}\left(\operatorname{cond}\left(\Psi \Phi^{2}\right) \operatorname{cond}(\Phi)\right)\right)$. Since $\mathbf{f}$ is a newform of level $\mathcal{Q M}$ we have $\mathcal{Q M} \mid \widetilde{\mathcal{Q}}^{\prime} \mathcal{M}^{\prime}$. Thus $\mathcal{M}=\mathcal{M}^{\prime}$ and $g_{\bar{\Phi}}=\sum_{\mathfrak{a} \mid \widetilde{\mathcal{Q}}^{\prime} \mathcal{Q}^{-1}} c_{\mathfrak{a}} \mathbf{f} \mid B_{\mathfrak{a}}$. Finally,

$$
\mathbf{g}-\mathbf{g}\left|T_{\mathfrak{q}}\right| B_{\mathfrak{q}}=\left(\mathbf{g}_{\bar{\Phi}}\right)_{\Phi}=\sum_{\mathfrak{a} \mid \widetilde{\mathcal{Q}}^{\prime} \mathcal{Q}^{-1}}\left(c_{\mathfrak{a}} \mathbf{f} \mid B_{\mathfrak{a}}\right)_{\Phi}=c_{\mathcal{O}} \mathbf{f}_{\Phi}
$$

Comparing first (i.e., $\mathcal{O}$-th) Fourier coefficients yields $c_{\mathcal{O}}=1$.

Corollary 5.9. If $\operatorname{ord}_{\mathfrak{q}}\left(\operatorname{cond}\left(\Psi \Phi^{2}\right)\right)<\operatorname{ord}_{q} \widetilde{\mathcal{Q}}$, then $\mathbf{f}_{\Phi}$ is a newform if and only if $\mathfrak{q}^{2} \mid \widetilde{\mathcal{Q}}$.

Proof. By Theorem 5.8, $\mathbf{f}_{\Phi}$ is a newform if and only if $\mathbf{g} \mid T_{\mathfrak{q}}=0$. Since $\mathbf{g}$ is a newform, $\mathbf{g} \mid T_{\mathfrak{q}}=0$ if and only if $C(\mathfrak{q}, \mathbf{g})=0$. The result now follows by Theorem $3.3(3)$.

\section{6. q-PRIMITIVE NEWFORMS}

In this section we obtain most of our results concerning when twists of newforms are newforms, and we consider the question of when a newform can be expressed as the twist of a newform of "lower" level (i.e., the question of primitivity).

Throughout this section fix a normalized newform $\mathbf{f} \in \mathcal{S}_{k}(\mathcal{N}, \Psi)$ and a prime $\mathfrak{q} \mid \mathcal{N}$. Let $\mathcal{Q}$ be the $\mathfrak{q}$-primary part of $\mathcal{N}$ and write $\mathcal{N}=\mathcal{Q M}$ as before. We shall consider only Hecke characters $\Phi$ whose conductor is a power of $\mathfrak{q}$. The character 
$\Phi_{\mathfrak{q}}$ is the restriction of the Hecke character $\Phi$ to the $\mathfrak{q}$-part of the ideles, but may be viewed as a numerical character on $(\mathcal{O} / \operatorname{cond}(\Phi))^{\times}$. Put

$$
\mathcal{Q}_{\Phi}^{\prime}=\operatorname{lcm}\left(\mathcal{Q}, \operatorname{cond}\left(\psi_{\mathfrak{q}}\right) \operatorname{cond}(\Phi), \operatorname{cond}(\Phi)^{2}\right) .
$$

Theorem 6.1. Let $\Phi$ be a Hecke character with $\mathfrak{q}$-primary conductor. If $\mathcal{Q} \mid \operatorname{cond}(\Phi)$ and $\operatorname{cond}\left(\psi_{\mathcal{Q}} \Phi\right)=\operatorname{cond}(\Phi)$, then $\mathbf{f}_{\Phi}$ is a newform in $\mathcal{S}_{k}\left(\mathcal{Q}_{\Phi}^{\prime} \mathcal{M}, \Psi \Phi^{2}\right)$. Moreover, if $\Psi_{1}$ is a Hecke character extending $\psi_{\mathcal{Q}} \Phi_{\mathfrak{q}}^{2}$ (viewing $\Phi_{\mathfrak{q}}$ as a numerical character), then $\lambda_{\mathcal{Q}, \Psi_{1}}\left(\mathbf{f}_{\Phi}\right)=\Phi_{\mathfrak{q}}(-1) \bar{\Psi}_{1}\left(\widetilde{t}_{\mathfrak{d}}\right) \tau\left(\Psi_{1} \bar{\Phi}\right) \tau(\bar{\Phi})^{-1}$.

Proof. Put $\mathcal{Q}^{\prime}=\mathcal{Q}_{\Phi}^{\prime}$ and $\mathcal{Q}^{\prime \prime}=\operatorname{cond}(\Phi)$. By Theorem $5.7(2 \mathrm{~b})$, the exact level of $\mathbf{f}_{\Phi}$ is $\mathcal{Q}^{\prime} \mathcal{M}$, and $\mathcal{Q}^{\prime}=\left(\mathcal{Q}^{\prime \prime}\right)^{2}$. Let $y \in G_{A}, y_{0}=\left(\begin{array}{ll}\tilde{a} & \tilde{b} \\ \tilde{c} & \widetilde{d}\end{array}\right)_{0}$ represent $W_{\mathcal{Q}^{\prime}}\left(\Psi_{1}\right)=$ $W_{\mathcal{Q}^{\prime}}^{\mathcal{Q}^{\prime} \mathcal{M}}\left(\Psi_{1}\right)$. Then

$$
\begin{array}{r}
\mathbf{f}_{\Phi} \mid W_{\mathcal{Q}^{\prime}}\left(\Psi_{1}\right)(x)=\overline{\Psi_{1}}(\operatorname{det} x) \overline{\psi_{\mathfrak{q}} \Phi_{\mathfrak{q}}^{2}}\left(\widetilde{b}_{\mathfrak{d}}\right) \overline{\psi_{\mathcal{M}}}(\widetilde{a}) \tau(\bar{\Phi})^{-1} \Phi(\operatorname{det}(x y)) \times \\
\sum_{\nu \in\left(\mathcal{Q}^{\prime \prime} \mathfrak{d}\right)^{-1} / \mathfrak{d}^{-1}} \bar{\Phi}_{\infty}(\nu) \bar{\Phi}^{*}\left(\nu \mathcal{Q}^{\prime \prime} \mathfrak{d}\right) \mathbf{f}\left|\left(\begin{array}{ll}
1 & \nu \\
0 & 1
\end{array}\right)_{0}\right| y(x)
\end{array}
$$

We may assume that $\left(\nu \mathcal{Q}^{\prime \prime} \mathfrak{d}, \mathfrak{q}\right)=1$, so for each such $\nu$ there exists a unique $\nu^{\prime} \in\left(\mathcal{Q}^{\prime \prime} \mathfrak{d}\right)^{-1} / \mathfrak{d}^{-1}$ such that $\left(\nu \nu^{\prime} \widetilde{c}-\widetilde{b}\right) \mathcal{O} \in \mathcal{Q}^{\prime \prime} \mathfrak{d}^{-1}$. Then

$$
\left(\begin{array}{ll}
1 & \nu \\
0 & 1
\end{array}\right)_{0}\left(\begin{array}{ll}
\widetilde{a} & \widetilde{b} \\
\widetilde{c} & \widetilde{d}
\end{array}\right)_{0}\left(\begin{array}{cc}
1 & -\nu^{\prime} \\
0 & 1
\end{array}\right)_{0}=\widetilde{q} w
$$

where $\widetilde{q} \in K_{A}^{\times}$with $\widetilde{q} \mathcal{O}=\mathcal{Q}^{\prime \prime}$ and $\widetilde{q}_{\infty}=1$, and where $w \in W\left(\mathcal{Q}^{\prime \prime} \mathcal{M}\right) \subset W(\mathcal{N})$. Thus

$$
\mathbf{f}\left|\left(\begin{array}{ll}
1 & \nu \\
0 & 1
\end{array}\right)_{0}\right| y(x)=\mathbf{f} \mid \widetilde{q} w\left(\begin{array}{ll}
1 & \nu^{\prime} \\
0 & 1
\end{array}\right)_{0}(x) .
$$

A straightforward but tedious computation permits us to rewrite the sum over $\nu$ as a sum over $\nu^{\prime}$. The end result is

$$
\mathbf{f}_{\Phi} \mid W_{\mathcal{Q}^{\prime}}\left(\Psi_{1}\right)=\Phi_{\mathfrak{q}}(-1) \bar{\Psi}_{1}\left(t_{\mathfrak{d}}\right) \tau\left(\Psi_{1} \bar{\Phi}\right) \tau(\bar{\Phi})^{-1} \mathbf{f}_{\bar{\Psi}_{1} \Phi}
$$

which confirms the claim about the pseudo-eigenvalue.

By Theorem 5.8, there is a normalized newform $\mathbf{g} \in \mathcal{S}_{k}\left(\widetilde{\mathcal{Q}} \mathcal{M}, \Psi \Phi^{2}\right)$ such that $\widetilde{\mathcal{Q}}$ is $\mathfrak{q}$-primary and $\mathbf{f}_{\Phi}=\mathbf{g}-\mathbf{g}\left|T_{\mathfrak{q}}\right| B_{\mathfrak{q}}$. The exact level of $\mathbf{g}-\mathbf{g}\left|T_{\mathfrak{q}}\right| B_{\mathfrak{q}}$ is $\mathfrak{q} \widetilde{\mathcal{Q}} \mathcal{M}$ or $\widetilde{\mathcal{Q}} \mathcal{M}$ (the later case if $C(\mathfrak{q}, \mathbf{g})=0)$. Thus $\left(\mathcal{Q}^{\prime \prime}\right)^{2}=\widetilde{\mathcal{Q}}$ or $\mathfrak{q} \widetilde{\mathcal{Q}}$. By Corollary 5.9, $\mathbf{f}_{\Phi}$ must be a newform unless $\mathcal{Q}^{\prime \prime}=\mathcal{Q}=\widetilde{\mathcal{Q}}=\mathfrak{q}$, so we assume this later condition and derive a contradiction.

From the first part of the proof we have that

$$
\Phi_{\mathfrak{q}}(-1) \bar{\Psi}_{1}\left(t_{\mathfrak{d}}\right) \tau\left(\Psi_{1} \bar{\Phi}\right) \mathbf{f}_{\bar{\Psi}_{1} \Phi}=\tau(\bar{\Phi}) \mathbf{f}_{\Phi}\left|W_{\mathcal{Q}^{\prime}}\left(\Psi_{1}\right)=\tau(\bar{\Phi})\left(\mathbf{g}-\mathbf{g}\left|T_{\mathfrak{q}}\right| B_{\mathfrak{q}}\right)\right| W_{\mathfrak{q}^{2}}\left(\Psi_{1}\right)
$$

Also,

$$
\mathbf{g}\left|W_{\mathfrak{q}^{2}}\left(\Psi_{1}\right)=\mathbf{g}\right| W_{\mathfrak{q}}\left(\Psi_{1}\right)\left(\begin{array}{ll}
\widetilde{q} & 0 \\
0 & 1
\end{array}\right)=N(\mathfrak{q})^{k_{0} / 2}\left(\Psi \bar{\Psi}_{1}^{2} \Phi^{2}\right)(\widetilde{q}) \mathbf{g}\left|W_{\mathfrak{q}}\left(\Psi_{1}\right)\right| B_{\mathfrak{q}}
$$

and

$$
\mathbf{g}\left|T_{\mathfrak{q}}\right| B_{\mathfrak{q}}\left|W_{\mathfrak{q}^{2}}\left(\Psi_{1}\right)=C(\mathfrak{q}, \mathbf{g}) N(\mathfrak{q})^{-k_{0} / 2} \mathbf{g}\right| W_{\mathfrak{q}}\left(\Psi_{1}\right)
$$


Note that by Theorem $3.3, C(\mathfrak{q}, \mathbf{g}) \neq 0$. Taking $\widetilde{\mathbf{g}}$ to be the normalized newform such that $\mathbf{g} \mid W_{\mathfrak{q}}\left(\Psi_{1}\right)=\lambda_{\mathfrak{q}}(\mathbf{g}) \widetilde{\mathbf{g}}$, we have

$\Phi_{\mathfrak{q}}(-1) \bar{\Psi}_{1}\left(t_{\mathfrak{o}}\right) \tau\left(\Psi_{1} \bar{\Phi}\right) \mathbf{f}_{\bar{\Psi}_{1} \Phi}=\tau(\bar{\Phi}) C(\mathfrak{q}, \mathbf{g}) N(\mathfrak{q})^{-k_{0} / 2} \lambda_{\mathfrak{q}}(\mathbf{g})\left(\widetilde{\mathbf{g}}-C(\mathfrak{q}, \mathbf{g})^{-1} N(\mathfrak{q})^{k_{0}} \widetilde{\mathbf{g}} \mid B_{\mathfrak{q}}\right)$.

Comparing the absolute values of the $\mathcal{O}$-th Fourier coefficients of both sides yields

$$
N(\mathfrak{q})^{1 / 2}=N(\mathfrak{q})^{1 / 2}|C(\mathfrak{q}, \mathbf{g})| N(\mathfrak{q})^{-k_{0} / 2}\left|\lambda_{\mathfrak{q}}(\mathbf{g})\right| .
$$

From Theorem $4.2,\left|\lambda_{\mathfrak{q}}(\mathbf{g})\right|=1$, which shows that $|C(\mathfrak{q}, \mathbf{g})|=N(\mathfrak{q})^{k_{0} / 2}$, contrary to Theorem 3.3. Therefore, $\mathbf{f}_{\Phi}$ is a newform.

The following corollary is an immediate consequence of the theorem above.

Corollary 6.2. If $\mathcal{Q}=\mathfrak{q}$ and $\operatorname{ord}_{\mathfrak{q}}(\operatorname{cond}(\Psi))=0$ then the twist of $\mathbf{f}$ by any nontrivial $\mathfrak{q}$-primary Hecke character $\Phi$ is a normalized newform of level $\mathcal{Q}_{\Phi}^{\prime} \mathcal{M}$.

Theorem 6.3. Suppose that $\operatorname{cond}\left(\psi_{\mathcal{Q}}\right)=\mathcal{Q}$ and $\operatorname{ord}_{\mathfrak{q}}(\operatorname{cond}(\Phi))<\operatorname{ord}_{\mathfrak{q}}(\mathcal{Q})$. If $C(\mathfrak{q}, \mathbf{f}) \neq 0$ then $\mathbf{f}_{\Phi}$ is a normalized newform in $\mathcal{S}_{k}\left(\mathcal{N} \operatorname{cond}(\Phi), \Psi \Phi^{2}\right)$.

Remark. The condition $C(\mathfrak{q}, \mathbf{f}) \neq 0$ is discussed more fully in the remark following Theorem 3.3.

Proof. Put $\mathcal{Q}^{\prime \prime}=\operatorname{cond}(\Phi)$ and write $\mathcal{N}=\mathcal{Q M}$ as usual. By Proposition 5.2, $\mathbf{f}_{\Phi}$ is an eigenform for all Hecke operators $T_{\mathfrak{p}}$ with $\mathfrak{p} \neq \mathfrak{q}$. We observe that $C\left(\mathfrak{m}, \mathbf{f}_{\Phi} \mid T_{\mathfrak{q}}\right)=$ $\Phi^{*}(\mathfrak{m} \mathfrak{q}) C(\mathfrak{m} \mathfrak{q}, \mathbf{f})=0$, so $\mathbf{f}_{\Phi}$ is an eigenform for Hecke operators $T_{\mathfrak{p}}$ for all primes $\mathfrak{p}$. By Theorem $5.7(2 \mathrm{~b}), \mathbf{f}_{\Phi}$ has exact level $\mathcal{N} \mathcal{Q}^{\prime \prime}$, so by Theorem 3.7, we need only show that $\mathbf{f}_{\Phi}$ is an eigenform for the operator $H_{\mathcal{N} \mathcal{Q}^{\prime \prime}} K$.

Consider $\left(\mathbf{f}\left|T_{\mathcal{Q}^{\prime \prime}}\right| H_{\mathcal{N}}\right)_{\bar{\Phi}} \mid H_{\mathcal{N} \mathcal{Q}^{\prime \prime}}^{-1}$. A straightforward but tedious calculation completely analogous to Theorem 4.2 of [1] yields that

$\left(\mathbf{f}\left|T_{\mathcal{Q}^{\prime \prime}}\right| H_{\mathcal{N}}\right)_{\bar{\Phi}}\left|K=\left(\mathbf{f}\left|T_{\mathcal{Q}^{\prime \prime}}\right| H_{\mathcal{N}}\right)_{\bar{\Phi}}\right| H_{\mathcal{N} \mathcal{Q}^{\prime \prime}}^{-1}\left|H_{\mathcal{N} \mathcal{Q}^{\prime \prime}}\right| K=\overline{C\left(\mathcal{Q}^{\prime \prime}, \mathbf{f}\right)}\left(\mathbf{f} \mid H_{\mathcal{N}}\right)_{\bar{\Phi}} \mid K$

By Proposition 5.3, $\mathbf{f}_{\Phi} \mid K=(\mathbf{f} \mid K)_{\bar{\Phi}}$ and since $\mathbf{f}\left|H_{\mathcal{N}}\right| K=\gamma \mathbf{f}$ for $|\gamma|=1$, we have

$$
\left(\mathbf{f}\left|T_{\mathcal{Q}^{\prime \prime}}\right| H_{\mathcal{N}}\right)_{\bar{\Phi}} \mid K=\overline{C\left(\mathcal{Q}^{\prime \prime}, \mathbf{f}\right)} \gamma \mathbf{f}_{\Phi} .
$$

Since $\mathbf{f}\left|H_{\mathcal{N} \mathcal{Q}^{\prime \prime}}\right| K=(-1)^{k} \mathbf{f}|K| H_{\mathcal{N} \mathcal{Q}^{\prime \prime}}, \mathbf{f}\left|H_{\mathcal{N} \mathcal{Q}^{\prime \prime}}\right| H_{\mathcal{N} \mathcal{Q}^{\prime \prime}}=(-1)^{k} \mathbf{f}$, and $\mathbf{f}|K| K=\mathbf{f}$ we have

$$
\left(\mathbf{f}\left|T_{\mathcal{Q}^{\prime \prime}}\right| H_{\mathcal{N}}\right)_{\bar{\Phi}}\left|K=\left(\mathbf{f}\left|T_{\mathcal{Q}^{\prime \prime}}\right| H_{\mathcal{N}}\right)_{\bar{\Phi}}\right| H_{\mathcal{N} \mathcal{Q}^{\prime \prime}}^{-1}\left|H_{\mathcal{N} \mathcal{Q}^{\prime \prime}}\right| K=\overline{C\left(\mathcal{Q}^{\prime \prime}, \mathbf{f}\right)} \gamma \mathbf{f}_{\Phi},
$$

from which it follows that $\mathbf{f}_{\Phi}$ is an eigenform for $H_{\mathcal{N} \mathcal{Q}^{\prime \prime}} K$ and hence is a newform of level $\mathcal{N} \mathcal{Q}^{\prime \prime}$ provided $C\left(\mathcal{Q}^{\prime \prime}, \mathbf{f}\right)=C(\mathfrak{q}, \mathbf{f})^{\operatorname{ord}_{\mathfrak{q}}\left(\mathcal{Q}^{\prime \prime}\right)} \neq 0$, which has been assumed.

From this we get

Corollary 6.4. Suppose that $\operatorname{cond}\left(\psi_{\mathcal{Q}}\right)=\mathcal{Q}, \Phi$ is nontrivial, and $C(\mathfrak{q}, \mathbf{f}) \neq 0$. Put

$$
\widetilde{Q}= \begin{cases}\operatorname{cond}(\Phi) & \text { if } \operatorname{ord}_{\mathfrak{q}}(\operatorname{cond}(\Psi \Phi)) \geq \operatorname{ord}_{\mathfrak{q}}(\mathcal{Q}) \\ \operatorname{cond}\left(\psi_{\mathcal{Q}} \Phi_{\mathfrak{q}}\right) & \text { if } \psi_{\mathcal{Q}} \Phi_{\mathfrak{q}} \neq 1 \text { and } \operatorname{ord}_{\mathfrak{q}}(\operatorname{cond}(\Psi \Phi))<\operatorname{ord}_{\mathfrak{q}}(\mathcal{Q}) .\end{cases}
$$


Then $\mathbf{f}_{\Phi}$ is a normalized newform in $\mathcal{S}_{k}\left(\widetilde{Q} \mathcal{N}, \Psi \Phi^{2}\right)$ if $\Phi$ does not extend $\bar{\psi}_{\mathcal{Q}}$. If $\Phi$ does extend $\bar{\psi}_{\mathcal{Q}}$ then $\mathbf{f}_{\Phi}$ is not a newform of any level.

Remark. The condition $C(\mathfrak{q}, \mathbf{f}) \neq 0$ is not required for the case where $\Phi$ does not extend $\bar{\psi}_{\mathcal{Q}}$ and $\operatorname{ord}_{\mathfrak{q}}(\operatorname{cond}(\Psi \Phi)) \geq \operatorname{ord}_{\mathfrak{q}}(\mathcal{Q})$. See also the remark following Thoerem 3.3 .

Proof. Let $\Psi_{\mathcal{Q}}$ be a Hecke character extending $\psi_{\mathcal{Q}}$, and consider $\mathbf{f}_{\bar{\Psi}_{\mathcal{Q}}}$. Let $\mathbf{g} \in$ $\mathcal{S}_{k}\left(\mathcal{N}, \Psi \bar{\Psi}_{\mathcal{Q}}^{2}\right)$ be the normalized newform such that $\mathbf{f} \mid W_{\mathcal{Q}}\left(\Psi_{\mathcal{Q}}\right)=\lambda_{\mathcal{Q}, \Psi_{\mathcal{Q}}}(\mathbf{f}) \mathbf{g}$. From Lemma 4.3, we know that for any prime $\mathfrak{p} \nmid \mathcal{Q}$ we have $C(\mathfrak{p}, \mathbf{g})=\bar{\Psi}_{\mathcal{Q}}^{*}(\mathfrak{p}) C(\mathfrak{p}, \mathbf{f})$, and $C(\mathfrak{q}, \mathbf{g})=\left(\Psi \Psi_{\mathcal{Q}}^{-1}\right)^{*}(\mathfrak{q}) \overline{C(\mathfrak{q}, \mathbf{f})}$. Moreover, for $\mathfrak{p} \nmid \mathcal{Q}, C\left(\mathfrak{p}, \mathbf{f}_{\bar{\Psi}_{\mathcal{Q}}}\right)=\bar{\Psi}_{\mathcal{Q}}^{*}(\mathfrak{p}) C(\mathfrak{p}, \mathbf{f})$, thus $\mathbf{g} \sim \mathbf{f}_{\bar{\Psi}_{\mathcal{Q}}}$. By Theorem 5.8, $\mathbf{f}_{\bar{\Psi}_{\mathcal{Q}}}=\mathbf{h}-\mathbf{h}\left|T_{\mathfrak{q}}\right| B_{\mathfrak{q}}$ where $\mathbf{h}$ is a normalized newform in $\mathcal{S}_{k}\left(\mathcal{Q}^{\prime} \mathcal{M}, \Psi \bar{\Psi}_{\mathcal{Q}}^{2}\right)$ for $\mathcal{Q}^{\prime}$ a power of $\mathfrak{q}$, so $\mathbf{f}_{\bar{\Psi}_{\mathcal{Q}}} \sim \mathbf{h}$, and hence $\mathbf{g} \sim \mathbf{h}$. By Theorem $3.5, \mathbf{g}=\mathbf{h}$, so $\mathbf{f}_{\bar{\Psi}_{\mathcal{Q}}}=\mathbf{g}-\mathbf{g}\left|T_{\mathfrak{q}}\right| B_{\mathfrak{q}} \neq \mathbf{g}$ since $C(\mathfrak{q}, \mathbf{g})=\left(\Psi \Psi_{\mathcal{Q}}^{-1}\right)^{*}(\mathfrak{q}) C(\mathfrak{q}, \mathbf{f}) \neq 0$, hence $\mathbf{f}_{\bar{\Psi}_{\mathcal{Q}}}$ is not a newform.

Now suppose that $\Phi \neq \bar{\Psi}_{\mathcal{Q}}$. Consider first the case where $\operatorname{ord}_{\mathfrak{q}}(\operatorname{cond}(\Psi \Phi)) \geq$ $\operatorname{ord}_{\mathfrak{q}}(\mathcal{Q})$. If $\operatorname{ord}_{\mathfrak{q}}(\operatorname{cond}(\Phi))<\operatorname{ord}_{\mathfrak{q}}(\mathcal{Q})=\operatorname{ord}_{\mathfrak{q}}(\operatorname{cond}(\Psi))$ then Theorem 6.3 yields the result. If $\operatorname{ord}_{\mathfrak{q}}(\operatorname{cond}(\Phi))=\operatorname{ord}_{\mathfrak{q}}(\operatorname{cond}(\Psi))$, then $\operatorname{cond}\left(\psi_{\mathcal{Q}} \Psi_{\mathfrak{q}}\right) \mid \mathcal{Q}$, which combined with the hypothesis of the theorem gives $\operatorname{cond}\left(\psi_{\mathcal{Q}} \Psi_{\mathfrak{q}}\right)=\mathcal{Q}=\operatorname{cond}\left(\psi_{\mathcal{Q}}\right)=\operatorname{cond}(\Phi)$. If $\operatorname{ord}_{\mathfrak{q}}(\operatorname{cond}(\Phi))>\operatorname{ord}_{\mathfrak{q}}(\operatorname{cond}(\Psi))$, then $\operatorname{cond}\left(\psi_{\mathcal{Q}} \Psi_{\mathfrak{q}}\right)=\operatorname{cond}(\Phi)$ of which $\mathcal{Q}$ is a proper divisor. In either of the later two cases, the result follows from Theorem 6.1. Next, suppose that $\operatorname{ord}_{\mathfrak{q}}(\operatorname{cond}(\Psi \Phi))<\operatorname{ord}_{\mathfrak{q}}(\mathcal{Q})$. Then $\Phi=\bar{\Psi}_{\mathcal{Q}} \Phi^{\prime}$ with $\Phi^{\prime} \neq 1$ and $\operatorname{ord}_{\mathfrak{q}}\left(\Phi^{\prime}\right)<\operatorname{ord}(\mathcal{Q})$. Let $\mathrm{g} \in \mathcal{S}_{k}\left(\mathcal{N}, \Psi \bar{\Psi}_{\mathcal{Q}}^{2}\right)$ be the normalized newform with $\mathbf{f} \mid W_{\mathcal{Q}}\left(\bar{\Psi}_{\mathcal{Q}}\right)=\lambda_{\mathcal{Q}, \bar{\Psi}_{\mathcal{Q}}}(\mathbf{f}) \mathbf{g}$. Then $\mathbf{f}_{\bar{\Psi}_{\mathcal{Q}}}=\mathbf{g}-\mathbf{g}\left|T_{\mathfrak{q}}\right| B_{\mathfrak{q}}$ (as above), so $\mathbf{f}_{\Phi}=\left(\mathbf{f}_{\bar{\Psi}_{\mathcal{Q}}}\right)_{\Phi^{\prime}}=$ $\mathbf{g}_{\Phi^{\prime}}$ is a normalized newform of level $\widetilde{Q} \mathcal{N}$ by Theorem 6.3.

In strict analogy with [1], we introduce the notion of a primitive newform.

Definition. Suppose that the prime $\mathfrak{q}$ divides $\mathcal{N}$. A newform $\mathbf{g} \in \mathcal{S}_{k}(\mathcal{N}, \Psi)$ is said to be $\mathfrak{q}$-primitive if $\mathbf{g}$ is not the twist of any newform of level $\mathcal{N}^{\prime}$ where $\mathcal{N}^{\prime}$ is a proper divisor of $\mathcal{N}$ by a Hecke character whose conductor is a power of $\mathfrak{q}$. Clearly $\mathbf{g}$ is $\mathfrak{q}$-primitive if $C(\mathfrak{q}, \mathbf{g}) \neq 0$.

Proposition 6.5. If $\mathbf{f}$ is a $\mathfrak{q}$-primitive newform and $C(\mathfrak{q}, \mathbf{f})=0$, then all twists of $\mathbf{f}$ by $\mathfrak{q}$-primary Hecke characters $\Phi$ are normalized newforms with level divisible by $\mathcal{N}$.

Proof. Let $\Phi$ be such a character. By Theorem 5.8, there exists a normalized newform $\mathbf{g} \in \mathcal{S}_{k}\left(\mathcal{Q}^{\prime} \mathcal{M}, \Psi \Phi^{2}\right)$ such that $\mathbf{f}_{\Phi}=\mathbf{g}-\mathbf{g}\left|T_{\mathfrak{q}}\right| B_{\mathfrak{q}}$. Since $C(\mathfrak{q}, \mathbf{f})=0$, we have $\mathbf{f}=\mathbf{g}_{\bar{\Phi}}=\mathbf{f}_{\Phi \bar{\Phi}}$. If $\mathbf{f}_{\Phi}$ is not a newform then $C(\mathfrak{q}, \mathbf{g}) \neq 0$, for if $C(\mathfrak{q}, \mathbf{g})=0$ then $\mathbf{f}_{\Phi}=\mathbf{g}-\mathbf{g}\left|T_{\mathfrak{q}}\right| B_{\mathfrak{q}}=\mathbf{g}-C(\mathfrak{q}, \mathbf{g}) \mathbf{g} \mid B_{\mathfrak{q}}=\mathbf{g}$ which would imply that $\mathbf{f}_{\Phi}$ is a newform, a contradicition. By Theorem $3.3, C(\mathfrak{q}, \mathbf{g}) \neq 0$ implies either that $\operatorname{cond}\left(\psi_{\mathcal{Q}} \Phi^{2}\right)=\mathcal{Q}^{\prime}$, or $\mathfrak{q} \| \mathcal{Q}^{\prime}$ and $\operatorname{ord}_{\mathfrak{q}}\left(\operatorname{cond}\left(\psi_{\mathcal{Q}} \Phi^{2}\right)\right)<\operatorname{ord}_{\mathfrak{q}}\left(\mathcal{Q}^{\prime}\right)$. In the first case, Coroallary 6.4 implies that $\mathbf{g}_{\bar{\Phi}}=\mathbf{f}$ has level $\mathcal{N}=\widetilde{\mathcal{Q}} \mathcal{Q}^{\prime} \mathcal{M}$; in particular, $\operatorname{ord}_{\mathfrak{q}}\left(\mathcal{Q}^{\prime}\right)<$ $\operatorname{ord}_{\mathfrak{q}}\left(\mathcal{Q}^{\prime} \widetilde{\mathcal{Q}}\right)=\operatorname{ord}_{q}(\mathcal{Q})$. In the second case, $\mathcal{Q}^{\prime}=\mathfrak{q}$ and $\operatorname{ord}_{\mathfrak{q}}\left(\operatorname{cond}\left(\psi_{\mathcal{Q}} \Phi^{2}\right)\right)=0$, so Corollary 6.2 implies that $\mathbf{g}_{\bar{\Phi}}=\mathbf{f}$ has level $\mathcal{N}=\operatorname{lcm}\left(\mathcal{Q}^{\prime}, \operatorname{cond}(\bar{\Phi}), \operatorname{cond}(\Phi)^{2}\right) \mathcal{M}$. In either case, it is clear that $\mathcal{Q}^{\prime} \mathcal{M}$ is a proper divisor of $\mathcal{N}$ and $\mathbf{f}=\mathbf{g}_{\bar{\Phi}}$ implies that 
$\mathbf{f}$ is not $\mathfrak{q}$-primitive, contrary to assumption. Therefore $\mathbf{f}_{\Phi}$ is a newform. The level of $\mathbf{f}_{\Phi}$ is divisible by $\mathcal{N}$ otherwise $\mathbf{f}=\left(\mathbf{f}_{\Phi}\right)_{\bar{\Phi}}$ would not be $\mathfrak{q}$-primitive.

Conversely, we have

Proposition 6.6. If all twists of $\mathbf{f}$ by Hecke characters $\Phi$ with $\mathfrak{q}$-primary conductors are newforms, then $\mathbf{f}$ is the twist of some $\mathfrak{q}$-primitive normalized newform.

Proof. Let $\Phi$ be a Hecke character with $\mathfrak{q}$-primary conductor such that the level of $\mathbf{f}_{\Phi}$ is minimal. That is $\mathbf{f}_{\Phi} \in \mathcal{S}_{k}\left(\mathcal{Q}^{\prime} \mathcal{M}, \Psi \Phi^{2}\right)$ with $\mathcal{Q}^{\prime}$ dividing the level of every twist of $\mathbf{f}$ by a Hecke character with $\mathfrak{q}$-primary conductor. By Theorem 5.8 there exists a normalized newform $\mathbf{g} \in \mathcal{S}_{k}\left(\widetilde{\mathcal{Q}} \mathcal{M}, \Psi \Phi^{2}\right)$ such that $\mathbf{f}_{\Phi}=\mathbf{g}-\mathbf{g}\left|T_{\mathfrak{q}}\right| B_{\mathfrak{q}}$. If $C(\mathfrak{q}, \mathbf{f}) \neq 0$, then $\mathbf{f}$ is $\mathfrak{q}$-primitive and hence is the twist by the trivial character of a $\mathfrak{q}$-primitive newform. Otherwise $C(\mathfrak{q}, \mathbf{f})=0$ and as in the previous proposition we have $\mathbf{f}=\mathbf{g}_{\bar{\Phi}}=\mathbf{f}_{\Phi \bar{\Phi}}$. Clearly $\mathbf{g}$ is $\mathfrak{q}$-primitive by minimality of $\mathcal{Q}^{\prime}$.

The proof of the following technical lemma is analogous to Lemma 4.11 of [1].

Lemma 6.7. Let $\Phi$ be a Hecke character with conductor dividing $\mathfrak{q}^{\beta}, \beta<\operatorname{ord}_{\mathfrak{q}}(Q)$. Let $\alpha=\operatorname{ord}_{\mathfrak{q}}(\Psi)$ and put

$$
\mathcal{Q}^{\prime}=\operatorname{lcm}\left(\mathcal{Q}, \mathfrak{q}^{\alpha+\beta}, \mathfrak{q}^{2 \beta}\right) \quad \mathcal{Q}^{\prime \prime}=\operatorname{lcm}\left(\mathfrak{q}, \mathfrak{q}^{\alpha-\beta} \mathcal{Q}, \mathfrak{q}^{-2 \beta} \mathcal{Q}^{2}\right) .
$$

Finally, let $\Psi_{\mathcal{Q}}$ be a Hecke character extending $\psi_{\mathcal{Q}}$. Then

$\mathbf{f}\left|R_{\Phi}\left(\mathfrak{q}^{\beta}\right)\right| W_{\mathcal{Q}^{\prime}}\left(\Psi_{\mathcal{Q}} \Phi^{2}\right)=\kappa \mathbf{f}\left|W_{\mathcal{Q}}\left(\Psi_{\mathcal{Q}}\right)\right| \sum_{\Phi_{1}} R_{\bar{\Phi}_{1}}\left(\mathcal{Q} \mathfrak{q}^{-\beta}\right)\left|W_{\mathcal{Q}^{\prime \prime}}\left(\overline{\Psi_{\mathcal{Q}} \Phi_{1}^{2}}\right)\right| R_{\bar{\Phi} \Psi_{\mathcal{Q}^{\Phi}}}\left(\mathcal{Q}^{\prime} \mathfrak{q}^{-\beta}\right)$

where $\kappa$ is a nonzero constant and where the sum is over Hecke characters $\Phi_{1}$, one for each numerical character defined on $\left(\mathcal{Q} / \mathfrak{q}^{\beta}\right)^{\times}$. The particular choice of the Hecke character extending the numerical characters is irrelevant but fixed.

Now we have

Theorem 6.8. Let $\alpha=\operatorname{ord}_{\mathfrak{q}}\left(\psi_{\mathcal{Q}}\right)$. If $(1 / 2) \operatorname{ord}_{\mathfrak{q}}(\mathcal{Q})<\alpha<\operatorname{ord}_{q}(\mathcal{Q})$ and $C(\mathfrak{q}, \mathbf{f})=$ 0 , then $\mathbf{f}$ is not $\mathfrak{q}$-primitive. Moreover, there exists a Hecke character $\Phi$ of conductor $\mathcal{Q} \mathfrak{q}^{-\alpha}$ and a normalized newform $\mathbf{g} \in \mathcal{S}_{k}\left(\mathfrak{q}^{\alpha} \mathcal{M}, \Psi \bar{\Phi}^{2}\right)$ such that $\mathbf{f}=\mathbf{g}_{\Phi}$.

Proof. The proof that $\mathbf{f}$ is not $\mathfrak{q}$-primitive is completely analogous to the first part of the proof of Theorem 4.3 of [1]. The proof of the second statement has minor variations which we indicate below. Since $\mathbf{f}$ is not $\mathfrak{q}$-primitive, there exists a Hecke character $\Phi$ of conductor $\mathfrak{q}^{\beta}$, a proper divisor $\widetilde{\mathcal{Q}}$ of $\mathcal{Q}$, and a $\mathfrak{q}$-primitive newform $\mathbf{g} \in \mathcal{S}_{k}\left(\widetilde{\mathcal{Q}} \mathcal{M}, \Psi \bar{\Phi}^{2}\right)$ such that $\mathbf{f}=\mathbf{g}_{\Phi}$. We claim that $\operatorname{ord}_{\mathfrak{q}}\left(\operatorname{cond}\left(\Psi \bar{\Phi}^{2}\right)\right)=\alpha$.

Since $\mathbf{f}=\mathbf{g}_{\Phi}$, we have $\mathbf{f}_{\bar{\Phi}}=\mathbf{g}_{\Phi \Phi}=\mathbf{g}-\mathbf{g}\left|T_{\mathfrak{q}}\right| B_{\mathfrak{q}} . \operatorname{Put} \mathcal{Q}^{\prime}=\operatorname{lcm}\left(\mathcal{Q}, \mathfrak{q}^{\alpha+\beta}, \mathfrak{q}^{2 \beta}\right)$ and $\gamma=\operatorname{ord}_{\mathfrak{q}}\left(\operatorname{cond}\left(\Psi \bar{\Phi}^{2}\right)\right)$. If $\gamma>\alpha$, then $\beta>\alpha$ and $\operatorname{ord}_{\mathfrak{q}}\left(\mathcal{Q}^{\prime}\right)=2 \beta>\operatorname{ord}_{\mathfrak{q}}(\mathcal{Q})$. By Theorem 5.7, $\mathbf{f}_{\bar{\Phi}}$ has exact level $\mathcal{Q}^{\prime} \mathcal{M}$ which implies $\mathcal{Q} \mid \widetilde{\mathcal{Q}}$, contrary to assumption. If $\gamma<\alpha$ then $\operatorname{cond}\left(\Phi^{2}\right)=\mathfrak{q}^{\alpha}$, so either $\beta=\alpha$ if $\mathfrak{q}$ is nondyadic or $\mathfrak{q}$ is dyadic of degree greater than 1 , or $\beta=\alpha+1$ if $\mathfrak{q}$ is a dyadic prime of degree 1 . In either case, $\operatorname{ord}_{\mathfrak{q}}(\mathcal{Q})<\operatorname{ord}_{\mathfrak{q}}\left(\mathcal{Q}^{\prime}\right)$ and $\operatorname{ord}_{\mathfrak{q}}(\operatorname{cond}(\Psi \bar{\Phi}))=\max (\alpha, \beta)$. Using Theorem 5.7 as above leads to a contradiction, so we conclude that $\operatorname{ord}_{\mathfrak{q}}\left(\operatorname{cond}\left(\Psi \bar{\Phi}^{2}\right)\right)=\alpha$ as claimed. 
Using arguments similar to those above, we see that $\operatorname{ord}_{\mathfrak{q}}\left(\operatorname{cond}\left(\Psi \bar{\Phi}^{2}\right)\right)=\alpha$ forces $\operatorname{cond}(\Phi)=\mathfrak{q}^{\beta} \mid \mathfrak{q}^{\alpha}$. Moreover, we claim that $\widetilde{\mathcal{Q}}=\mathfrak{q}^{\alpha}$. Now $\alpha>1$ by hypothesis, so if $\operatorname{ord}_{\mathfrak{q}}(\widetilde{\mathcal{Q}})>\alpha$, then $C(\mathfrak{q}, \mathbf{g})=0$ by Theorem 3.3. Moreover, $\operatorname{since} \operatorname{ord}_{\mathfrak{q}}(\widetilde{\mathcal{Q}})>\alpha>$ $(1 / 2) \operatorname{ord}_{\mathfrak{q}}(\mathcal{Q})>(1 / 2) \operatorname{ord}_{\mathfrak{q}}(\widetilde{\mathcal{Q}})$, the first part of this theorem implies that $\mathbf{g}$ is not $\mathfrak{q}$-primitive, contrary to asumption, hence $\widetilde{\mathcal{Q}}=\mathfrak{q}^{\alpha}$ as desired.

To complete the proof, we need only verify that $\mathfrak{q}^{\beta}=\mathcal{Q} \mathfrak{q}^{-\alpha}$. We know that $\beta \leq \alpha$. If $\beta<\alpha$, then $\mathfrak{q}^{\beta}=\mathcal{Q q}^{-\alpha}$ by Theorem 5.7 applied to $\mathbf{g}_{\Phi}$. Now assume that $\beta=\alpha$. If $\operatorname{ord}_{\mathfrak{q}}(\operatorname{cond}(\Psi \bar{\Phi}))=\alpha$, then by Theorem 5.7 again, we have $\mathcal{Q}=\mathfrak{q}^{2 \alpha}$, contrary to assumption, so $\operatorname{ord}_{\mathfrak{q}}(\operatorname{cond}(\Psi \bar{\Phi}))<\alpha$. This implies that $\Phi=\Psi_{\mathcal{Q}} \Phi^{\prime}$ where $\Psi_{\mathcal{Q}}$ is a Hecke character extending $\psi_{\mathcal{Q}}$ and where $\Phi^{\prime}$ is a $\mathfrak{q}$-primary Hecke character with $\operatorname{ord}_{\mathfrak{q}}\left(\operatorname{cond}\left(\Phi^{\prime}\right)\right)<\alpha$. Note that $\Phi^{\prime}$ is nontrivial, otherwise since $\mathbf{g}-\mathbf{g}\left|T_{\mathfrak{q}}\right| B_{\mathfrak{q}}=\mathbf{f}_{\Psi_{\mathcal{Q}}}$, and $\mathbf{f} \mid W_{\mathcal{Q}}\left(\Psi_{\mathcal{Q}}\right) \sim \mathbf{f}_{\overline{\Psi_{\mathcal{Q}}}} \sim \mathbf{g}$, we would have that $\mathbf{g}$ is a newform of level $\mathcal{N}$, contrary to assumption. Now let $\mathbf{g}^{\prime} \in \mathcal{S}_{k}\left(\mathfrak{q}^{\alpha} \mathcal{M}, \Psi \bar{\Psi}_{\mathcal{Q}}^{2}\right)$ be the normalized newform such that $\mathbf{g} \mid W_{\mathfrak{q}}{ }\left(\Psi_{\mathcal{Q}} \bar{\Phi}^{2}\right)=\lambda_{\mathfrak{q}^{\alpha}, \Psi_{\mathcal{Q}}} \bar{\Phi}^{2}(\mathbf{g}) \mathbf{g}^{\prime}$. Then as above, $\mathbf{g}_{\bar{\Psi}_{\mathcal{Q}} \Phi^{2}}=\mathbf{g}^{\prime}-\mathbf{g}^{\prime}|T-\mathfrak{q}| B_{\mathfrak{q}}$ and $\mathbf{f}=\mathbf{g}_{\Phi}=\mathbf{g}_{\Psi_{\mathcal{Q}} \bar{\Phi}}^{\prime}=\mathbf{g}_{\bar{\Phi}^{\prime}}^{\prime}$. By the same argument as above, we have $\operatorname{cond}\left(\Phi^{\prime}\right)=\mathcal{Q} \mathfrak{q}^{-\alpha}$.

As an immediate consequence of Theorems 6.8 and 3.3 we have

Corollary 6.9. If $\mathbf{f}$ is $\mathfrak{q}$-primitive and $C(\mathfrak{q}, \mathbf{f})=0$ then either $\operatorname{ord}_{\mathfrak{q}}\left(\operatorname{cond}\left(\psi_{\mathcal{Q}}\right)\right)<$ $(1 / 2) \operatorname{ord}_{\mathfrak{q}}(\mathcal{Q})$ or $\operatorname{ord}_{\mathfrak{q}}\left(\operatorname{cond}\left(\psi_{\mathcal{Q}}\right)\right)=\operatorname{ord}_{\mathfrak{q}}(\mathcal{Q}), \mathfrak{q}^{2} \mid \mathcal{Q}$ and the inertial degree of $\mathfrak{q}$ is greater than one.

Finally we have

\section{Theorem 6.10.}

(1) If $\mathcal{Q}=\mathfrak{q}^{2 \rho+1}$ and $\operatorname{cond}\left(\psi_{\mathcal{Q}}\right) \mid \mathfrak{q}^{\rho}$, then $\mathbf{f}$ is $\mathfrak{q}$-primitive.

(2) If $\mathcal{Q}=\mathfrak{q}^{2 \rho}$ where $\mathfrak{q}$ is a dyadic prime of degree one, and $\operatorname{cond}\left(\psi_{\mathcal{Q}}\right)=\mathfrak{q}^{\rho}$, then $\mathbf{f}$ is $\mathfrak{q}$-primitive.

(3) If $\mathfrak{q}$ is a dyadic prime of degree one, $\mathcal{Q}=\mathfrak{q}^{2 \rho}$ with $\rho \geq 2$ and $\operatorname{cond}\left(\psi_{\mathcal{Q}}\right) \mid$ $\mathfrak{q}^{\rho-1}$, then the exact level of $\mathbf{f}_{\Phi}$ divides $\mathcal{N q}^{-1}$ for any Hecke character $\Phi$ of conductor $\mathfrak{q}^{\rho}$. In particular, $\mathbf{f}$ is not $\mathfrak{q}$-primitive.

(4) If $\mathfrak{q}$ is a dyadic prime of degree one, $\mathcal{Q}=\mathfrak{q}^{2}$, and $\psi_{\mathcal{Q}}=1$, then $\mathbf{f}$ is $\mathfrak{q}$ primitive.

Proof. The proof has only minor variations to the proof of Theorem 4.4 in [1], but for completeness we sketch it.

Case 1) By Theorem 5.5, $\mathbf{f}$ is $\mathfrak{q}$-primitive if $\mathcal{Q}=\mathfrak{q}$ and $\psi_{\mathcal{Q}}=1$, so we may assume that $\rho \geq 1$. Suppose that $\operatorname{cond}\left(\psi_{\mathcal{Q}}\right) \mid \mathfrak{q}^{\rho}$ and $\mathbf{f}$ is not $\mathfrak{q}$-primitive. Then $\mathbf{f}=\mathbf{g}_{\Phi}$ for a $\mathfrak{q}$-primitive normalized newform $\mathbf{g} \in \mathcal{S}_{k}\left(\mathcal{Q}^{\prime} \mathcal{M}, \Psi \bar{\Phi}^{2}\right)$ where $\mathcal{Q}^{\prime}$ is a proper divisor of $\mathcal{Q}$ and $\Phi$ has $\mathfrak{q}$-primary conductor. Then $\mathbf{f}_{\bar{\Phi}}=\mathbf{g}-\mathbf{g}\left|T_{\mathfrak{q}}\right| B_{\mathfrak{q}}$. Since $\mathcal{Q}^{\prime}$ is a proper divisor of $\mathcal{Q}$, we must have that $\operatorname{cond}(\Phi) \mid \mathfrak{q}^{\rho}$ otherwise Theorem 5.7 would give $\mathcal{N}$ to be a proper divisor of the level of $\mathbf{f}=\mathbf{g}_{\Phi}$. On the other hand, if $\operatorname{cond}(\Phi) \mid \mathfrak{q}^{\rho}$ then Proposition 5.1 gives the level of $\mathbf{f}=\mathbf{g}_{\Phi}$ as a proper divisor of $\mathcal{N}$, contrary to assumption. Thus $\mathbf{f}$ is $\mathfrak{q}$-primitive.

Case 2) We have that $\mathcal{Q}=\mathfrak{q}^{2 \rho}$ where $\mathfrak{q}$ is a dyadic prime of degree one, and $\operatorname{cond}\left(\psi_{\mathcal{Q}}\right)=\mathfrak{q}^{\rho}$. If $\mathbf{f}$ is not $\mathfrak{q}$-primitive, then as above, $\mathbf{f}=\mathbf{g}_{\Phi}$ and $\mathbf{f}_{\bar{\Phi}}=\mathbf{g}-$ $\mathbf{g}\left|T_{\mathfrak{q}}\right| B_{\mathfrak{q}}$ with $\operatorname{cond}(\Phi)=\mathfrak{q}^{\rho}$. Note that since $\mathfrak{q}$ is a dyadic prime of degree one, 
$\operatorname{cond}\left(\Phi^{2}\right) \mid \mathfrak{q}^{\rho-1}$, hence $\operatorname{ord}_{\mathfrak{q}}\left(\operatorname{cond}\left(\Psi \bar{\Phi}^{2}\right)\right)=\rho>\operatorname{ord}_{\mathfrak{q}}\left(\mathcal{Q}^{\prime}\right)$. By Theorems 6.8 and 3.3, we have that $\mathcal{Q}^{\prime}=\mathfrak{q}^{\rho}$. Let $\Psi_{\mathcal{Q}}$ be a Hecke character extending $\psi_{\mathcal{Q}}$ and let $\mathbf{g}^{\prime} \in \mathcal{S}_{k}\left(\mathcal{Q}^{\prime} \mathcal{M}, \Psi{\overline{\Psi_{\mathcal{Q}}}}^{2} \Phi^{2}\right)$ be the normalized newform such that $\mathbf{g} \mid W_{\mathcal{Q}^{\prime}}\left(\Psi_{\mathcal{Q}} \bar{\Phi}^{2}\right)=$ $\lambda_{\mathcal{Q}^{\prime}, \Psi_{\mathcal{Q}} \bar{\Phi}^{2}}(\mathbf{g}) \mathbf{g}^{\prime}$. Writing $\Phi=\bar{\Psi}_{\mathcal{Q}} \Phi^{2} \Psi_{\mathcal{Q}} \bar{\Phi}$ where $\Psi_{\mathcal{Q}} \bar{\Phi}$ is a character of conductor $\mathfrak{q}^{\beta}$, $\beta<\rho$, we have $\mathbf{f}=\mathbf{g}_{\Phi}=\mathbf{g}_{\bar{\Psi}_{\mathcal{Q}} \Phi^{2} \Psi_{\mathcal{Q}} \bar{\Phi}}=\mathbf{g}_{\Psi_{\mathcal{Q}} \Phi}^{\prime}$ since $\mathbf{g}^{\prime}=\mathbf{g}_{\bar{\Psi}_{\mathcal{Q}} \Phi^{2}}=\mathbf{g}^{\prime}-\mathbf{g}^{\prime}\left|T_{\mathfrak{q}}\right| B_{\mathfrak{q}}$. Here $\Psi_{\mathcal{Q}} \bar{\Phi}$ has nontrivial conductor since $\mathbf{f}$ is a newform and $\mathbf{g}^{\prime}$ and $\mathbf{f}$ have different levels. It follows from Theorem 6.3 - notice that we again use that the degree of $\mathfrak{q}$ is one (see Thoerem $3.3 \mathrm{ii}$ - that $\mathbf{g}_{\Psi_{\mathcal{Q}} \Phi}^{\prime}=\mathbf{f}$ has exact level $\mathcal{Q}^{\prime} \mathcal{M} \mathfrak{q}^{\beta}$ which is a proper divisor of $\mathcal{N}=\mathcal{Q M}$, a contradiction. Thus $\mathbf{f}$ is $\mathfrak{q}$-primitive.

Case 3) Here we assume that $\mathfrak{q}$ is a dyadic prime of degree one, $\mathcal{Q}=\mathfrak{q}^{2 \rho}$ with $\rho \geq 2$ and $\operatorname{cond}\left(\psi_{\mathcal{Q}}\right) \mid \mathfrak{q}^{\rho-1}$. Let $\Phi$ be a Hecke character of conductor $\mathfrak{q}^{\rho}$. By Proposition 5.1, the exact level of $\mathbf{f}_{\Phi}$ divides $\mathcal{N}$. Let $\left(\begin{array}{l}\tilde{a} \widetilde{b} \\ \widetilde{c} \tilde{d}\end{array}\right) \in W\left(\mathcal{N q}^{-1}\right)-W(\mathcal{N})$. In a computation completely analogous to the proof in [1], and using Theorem 3.4, we deduce that $\mathbf{f}_{\Phi} \mid\left(\begin{array}{l}\tilde{a} \widetilde{b} \\ \widetilde{c}\end{array}\right)=\psi \Phi_{\mathfrak{q}}(\widetilde{a}) \mathbf{f}_{\Phi}$, which implies the result. Note that once again we have used that the degree of $\mathfrak{q}$ is one since in this case $\left[W\left(\mathcal{N} \mathfrak{q}^{-1}\right)\right.$ : $W(\mathcal{N})]=N(\mathfrak{q})=2$.

Case 4) This follows easily from Theorem 6.1 and from the fact that there are no numerical characters of conductor $\mathfrak{q}$ since $\mathfrak{q}$ is a dyadic prime of degree one.

\section{Some Applications}

We now present two applications of the preceding results. The first application which we give strengthens Theorem 5.7 from a result characterizing the exact level of a twist to a theorem telling us that the twists described by Theorem 5.7 are newforms. The authors thank Arnold Pizer for suggesting this result.

Theorem 7.1. Let $\mathcal{N}$ be an integral ideal and let $\mathfrak{q}$ be a prime, $\mathfrak{q} \mid \mathcal{N}$. Write $\mathcal{N}=\mathcal{Q M}$ where $\mathcal{Q}$ is the q-primary part of $\mathcal{N}$. Take $\mathbf{f}$ to be a normalized newform in $\mathcal{S}_{k}(\mathcal{N}, \Psi)$ with $\operatorname{ord}_{\mathfrak{q}}(\operatorname{cond}(\Psi))=\alpha, \alpha \geq 0$. Let $\Phi$ be a character of conductor $\mathfrak{q}^{\beta}$, $\beta \geq 1$, and put $\mathcal{Q}^{\prime}=\operatorname{lcm}\left(\mathcal{Q}, \mathfrak{q}^{\alpha+\beta}, \mathfrak{q}^{2 \beta}\right)$. Then $\mathbf{f}_{\Phi}$ is a newform in $\mathcal{S}_{k}\left(\mathcal{Q}^{\prime} \mathcal{M}, \Psi \Phi^{2}\right)$ provided that

(1) $\max (\alpha+\beta, 2 \beta)<\operatorname{ord}_{\mathfrak{q}}(\mathcal{Q})$ if $\mathcal{Q}^{\prime}=\mathcal{Q}$, or

(2) $\operatorname{ord}_{\mathfrak{q}}(\operatorname{cond}(\Psi \Phi))=\max (\alpha, \beta)$ if $\operatorname{ord}_{\mathfrak{q}}\left(\mathcal{Q}^{\prime}\right)>\operatorname{ord}_{\mathfrak{q}}(\mathcal{Q})$ and $\mathfrak{q}$ is a prime of degree one.

Proof. By Theorem 5.8, $\mathbf{f}_{\Phi}=\mathbf{g}-\mathbf{g}\left|T_{\mathfrak{q}}\right| B_{\mathfrak{q}}$ where $\mathbf{g} \in \mathcal{S}_{k}\left(\widetilde{\mathcal{Q}} \mathcal{M}, \Psi \Phi^{2}\right)$ is a newform and $\widetilde{\mathcal{Q}}$ is $\mathfrak{q}$-primary. Since by Theorem $5.7, \mathbf{f}_{\Phi}$ has exact level $\mathcal{Q}^{\prime} \mathcal{M}$, it follows from Proposition 2.1 that $\mathcal{Q}^{\prime}=\widetilde{\mathcal{Q}}$ or $\mathcal{Q}^{\prime}=\mathfrak{q} \widetilde{\mathcal{Q}}$.

First suppose that $\mathcal{Q}^{\prime}=\mathcal{Q}$ and $\max (\alpha+\beta, 2 \beta)<\operatorname{ord}_{\mathfrak{q}}(\mathcal{Q})$. Since $\beta \geq 1$ and $2 \beta<\operatorname{ord}_{\mathfrak{q}}(\mathcal{Q})=\operatorname{ord}_{\mathfrak{q}}\left(\mathcal{Q}^{\prime}\right)$, we have $\mathfrak{q}^{3} \mid \mathcal{Q}^{\prime}$, hence $\mathfrak{q}^{2} \mid \widetilde{\mathcal{Q}}$. Moreover, we see that $\operatorname{ord}_{q}\left(\operatorname{cond}\left(\Psi \Phi^{2}\right)\right)<\operatorname{ord}_{q}(\widetilde{\mathcal{Q}})$ as follows: If $\alpha \leq \beta$, then $\operatorname{ord}_{q}\left(\operatorname{cond}\left(\Psi \Phi^{2}\right)\right) \leq \beta$. By hypothesis, $2 \beta<\operatorname{ord}_{q}\left(\mathcal{Q}^{\prime}\right)$, so $\beta+1<2 \beta<\operatorname{ord}_{q}\left(\mathcal{Q}^{\prime}\right)$ or $\beta<\operatorname{ord}_{\mathfrak{q}}\left(\mathcal{Q}^{\prime}\right)-1 \leq \operatorname{ord}_{\mathfrak{q}}(\widetilde{\mathcal{Q}})$. If $\alpha>\beta$, then $\operatorname{ord}_{q}\left(\operatorname{cond}\left(\Psi \Phi^{2}\right)\right)=\alpha$. By hypothesis, $\alpha+1 \leq \alpha+\beta<\operatorname{ord}_{\mathfrak{q}}(\mathcal{Q})=$ $\operatorname{ord}_{\mathfrak{q}}\left(\mathcal{Q}^{\prime}\right)$, hence $\alpha<\operatorname{ord}_{\mathfrak{q}}\left(\mathcal{Q}^{\prime}\right)-1 \leq \operatorname{ord}_{\mathfrak{q}}(\widetilde{\mathcal{Q}})$ as claimed. By Theorem 3.3iii, we have $\mathbf{g} \mid T_{\mathfrak{q}}=0$ hence $\mathbf{f}_{\Phi}=\mathbf{g}$ is a newform of level $\widetilde{\mathcal{Q}} \mathcal{M}$. As $\mathbf{f}_{\Phi}$ has exact level $\mathcal{Q}^{\prime} \mathcal{M}$, we have $\mathfrak{q}^{\prime}=\widetilde{\mathcal{Q}}$, and the result follows in this case. 
Next suppose that $\operatorname{ord}_{\mathfrak{q}}\left(\mathcal{Q}^{\prime}\right)>\operatorname{ord}_{\mathfrak{q}}(\mathcal{Q})$ and $\operatorname{ord}_{\mathfrak{q}}(\operatorname{cond}(\Psi \Phi))=\max (\alpha, \beta)$. We consider the cases $\alpha>\beta$ and $\alpha \leq \beta$ separately.

Case A. If $\alpha>\beta$, then $\operatorname{ord}_{q}\left(\operatorname{cond}\left(\Psi \Phi^{2}\right)\right)=\operatorname{ord}_{\mathfrak{q}}(\operatorname{cond}(\Psi \Phi))=\operatorname{ord}_{\mathfrak{q}}(\Psi)=\alpha$.

Subcase $1 . \mathfrak{q}^{2} \mid \widetilde{\mathcal{Q}}$.

If $\widetilde{\mathcal{Q}}=\mathcal{Q}^{\prime}$ then $\operatorname{ord}_{\mathfrak{q}}\left(\operatorname{cond}\left(\Psi \Phi^{2}\right)\right)<\operatorname{ord}_{\mathfrak{q}}(\widetilde{\mathfrak{q}})$, so by Corollary $5.9, \mathbf{f}_{\Phi}$ is a newform of level $\mathcal{Q}^{\prime} \mathcal{M}$, as desired. Otherwise, $\widetilde{\mathcal{Q}}=\mathcal{Q}^{\prime} \mathfrak{q}^{-1}$; we show that this can't happen. If $\widetilde{\mathcal{Q}}=\mathcal{Q}^{\prime} \mathfrak{q}^{-1}$, then $\operatorname{ord}_{\mathfrak{q}}\left(\operatorname{cond}\left(\Psi \Phi^{2}\right)\right)=\operatorname{ord}_{\mathfrak{q}}(\widetilde{\mathcal{Q}})$, for if $\operatorname{ord}_{\mathfrak{q}}\left(\operatorname{cond}\left(\Psi \Phi^{2}\right)\right)<\operatorname{ord}_{\mathfrak{q}}(\widetilde{\mathcal{Q}})$ then $\mathbf{f}_{\Phi}=\mathbf{g}-\mathbf{g}\left|T_{\mathfrak{q}}\right| B_{\mathfrak{q}}$ would have level $\widetilde{\mathcal{Q}}$ by Proposition 2.1, and since $\mathcal{Q}^{\prime} \mathcal{M}$ is the exact level, $\mathcal{Q}^{\prime} \mid \widetilde{\mathcal{Q}}=\mathcal{Q}^{\prime} \mathfrak{q}^{-1}$, a contradiction. Now $\operatorname{ord}_{\mathfrak{q}}\left(\operatorname{cond}\left(\Psi \Phi^{2}\right)\right)=\operatorname{ord}_{\mathfrak{q}}(\widetilde{\mathcal{Q}})$, $\mathfrak{q}$ a prime of degree one, and Theorem 5.8 imply that $\mathbf{f}_{\Phi}$ is not a newform of any level. From this we derive a contradicition.

Since $\operatorname{ord}_{\mathfrak{q}}\left(\operatorname{cond}\left(\Psi \Phi^{2}\right)\right)=\operatorname{ord}_{\mathfrak{q}}(\widetilde{\mathcal{Q}})$, we have

$$
\operatorname{ord}_{\mathfrak{q}}(\widetilde{\mathcal{Q}})=\operatorname{ord}_{\mathfrak{q}}\left(\operatorname{cond}\left(\Psi \Phi^{2}\right)\right)=\operatorname{ord}_{\mathfrak{q}}(\operatorname{cond}(\Psi \Phi))=\operatorname{ord}_{\mathfrak{q}}(\operatorname{cond}(\Psi))=\alpha \leq \operatorname{ord}_{\mathfrak{q}}(\mathcal{Q})
$$

If $\operatorname{ord}_{\mathfrak{q}}(\operatorname{cond}(\Psi))<\operatorname{ord}_{q}(\mathcal{Q})$ then $\operatorname{ord}_{\mathfrak{q}}(\widetilde{\mathcal{Q}})=\operatorname{ord}_{\mathfrak{q}}(\operatorname{cond}(\Psi)) \leq \operatorname{ord}_{\mathfrak{q}}\left(\mathcal{Q q}^{-1}\right)<$ $\operatorname{ord}_{\mathfrak{q}}\left(\mathcal{Q}^{\prime} \mathfrak{q}^{-1}\right)=\operatorname{ord}_{\mathfrak{q}}(\widetilde{\mathcal{Q}})$, a contradiction. Otherwise, $\operatorname{ord}_{\mathfrak{q}}(\operatorname{cond}(\Psi))=\operatorname{ord}_{q}(\mathcal{Q})$. Observe that $\Phi$ does not extend $\bar{\psi}_{\mathcal{Q}}$ since $\operatorname{ord}_{\mathfrak{q}}(\operatorname{cond}(\Psi \Phi))=\alpha>\beta \geq 1$, so by Corollary $6.4, \mathbf{f}_{\Phi}$ is a newform, a contradiction.

Subcase 2. $\widetilde{\mathcal{Q}}=\mathfrak{q}$.

If $\widetilde{\mathcal{Q}}=\mathcal{Q}^{\prime}$ then $1=\operatorname{ord}_{\mathfrak{q}}(\widetilde{\mathcal{Q}})=\operatorname{ord}_{\mathfrak{q}}\left(\mathcal{Q}^{\prime}\right)>\operatorname{ord}_{\mathfrak{q}}(\mathcal{Q}) \geq 1$, a contradicition. If $\widetilde{\mathcal{Q}}=\mathcal{Q}^{\prime} \mathfrak{q}^{-1}$, then $\operatorname{ord}_{\mathfrak{q}}\left(\mathfrak{q}^{\prime}\right)=2=\max (\alpha+\beta, 2 \beta)$, which implies $\beta=1$ and $\alpha=0,1$. But we are assuming that $\alpha>\beta$, a contradiction.

Case B. If $\alpha \leq \beta$, then $\operatorname{ord}_{\mathfrak{q}}\left(\operatorname{cond}(\Psi \Phi)=\beta\right.$ and $\operatorname{ord}_{\mathfrak{q}}\left(\operatorname{cond}\left(\Psi \Phi^{2}\right)\right) \leq \beta$.

Subcase 1. $\mathfrak{q}^{2} \mid \widetilde{\mathcal{Q}}$.

If $\widetilde{\mathcal{Q}}=\mathcal{Q}^{\prime}\left(=\mathfrak{q}^{2 \beta}\right)$, then $\operatorname{ord}_{\mathfrak{q}}\left(\operatorname{cond}\left(\Psi \Phi^{2}\right)\right)<\operatorname{ord}_{\mathfrak{q}}(\widetilde{\mathcal{Q}})$ and so by Corollary $5.9, \mathbf{f}_{\Phi}$ is a newform of level $\mathcal{Q}^{\prime} \mathcal{M}$. Otherwise, $\widetilde{\mathcal{Q}}=\mathcal{Q}^{\prime} \mathfrak{q}^{-1}$; we show that this can't happen. We must have $\operatorname{ord}_{\mathfrak{q}}\left(\operatorname{cond}\left(\Psi \Phi^{2}\right)\right)=\operatorname{ord}_{\mathfrak{q}}(\widetilde{\mathcal{Q}})$ otherwise by Theorem 3.3iii, $\mathbf{f}_{\Phi}$ is a newform of level $\widetilde{\mathcal{Q}} \mathcal{M}$ contradicting that its exact level is $\mathcal{Q}^{\prime} \mathcal{M}$. By Theorem 5.8 (since the degree of $\mathcal{Q}$ is one), $\mathbf{f}_{\Phi}$ is not a newform of any level. Now, $\beta=\operatorname{ord}_{\mathfrak{q}}(\operatorname{cond}(\Psi \Phi)) \geq \operatorname{ord}_{\mathfrak{q}}\left(\operatorname{cond}\left(\Psi \Phi^{2}\right)=\operatorname{ord}_{\mathfrak{q}}(\widetilde{\mathcal{Q}}) \operatorname{implies}\right.$ $2 \beta=\operatorname{ord}_{\mathfrak{q}}\left(\mathcal{Q}^{\prime}\right)=\operatorname{ord}_{\mathfrak{q}}(\mathfrak{q} \widetilde{\mathcal{Q}}) \leq \beta+1$ which in turn implies that $\beta=1$. Thus $\alpha=0,1, \mathcal{Q}^{\prime}=\mathfrak{q}^{2}$ and $\widetilde{\mathcal{Q}}=\mathfrak{q}=\mathcal{Q}$. Since $\beta=\operatorname{ord}_{\mathfrak{q}}(\operatorname{cond}(\Psi \Phi))=1, \Phi$ does not extend $\bar{\psi}_{\mathcal{Q}}$. If $\alpha=1$ then Corollary 6.4 implies that $\mathbf{f}_{\Phi}$ is a newform, while if $\alpha=0$, $\mathbf{f}_{\Phi}=\mathbf{g}-\mathbf{g}\left|T_{\mathfrak{q}}\right| B_{\mathfrak{q}}$ is a newform since $\mathbf{g} \mid T_{\mathfrak{q}}=0$ by Theorem 3.3iii. In either case, this provides the desired contradiction.

Subcase 2. $\widetilde{\mathcal{Q}}=\mathfrak{q}$.

If $\widetilde{\mathcal{Q}}=\mathcal{Q}^{\prime}$ then $\mathfrak{q}=\widetilde{\mathcal{Q}}=\mathcal{Q}^{\prime}=\mathfrak{q}^{2 \beta}$, a contradiction. If $\widetilde{\mathcal{Q}}=\mathcal{Q}^{\prime} \mathfrak{q}^{-1}$ then $\mathcal{Q}^{\prime}=\mathfrak{q}^{2}$ implies $\beta=1$. Then $\alpha=0,1$ give contradictions as above.

Our second application gives a decomposition of a space of newforms as a direct sum of twists of other spaces of newforms. (Such decomposition theorems were studied extensively in [3] where the predominant tool was the Eichler-Selberg trace formula.) Here we let $\mathcal{S}^{\Phi}$ denote the space of cusp forms consisting of $\left\{\mathbf{f}_{\Phi} \mid \mathbf{f} \in \mathcal{S}\right\}$ where $\mathcal{S}$ is a space of cusp forms and $\Phi$ is a Hecke character. 
Theorem 7.2. Let $\mathfrak{q}$ be a dyadic prime of degree one and $\mathcal{M}$ an integral ideal prime to $\mathfrak{q}$. Let $\nu$ be an even integer, $\nu=2 \rho \geq 4, \Phi$ a q-primary Hecke character of conductor $\mathfrak{q}^{\rho}$, and assume that $\operatorname{ord}_{\mathfrak{q}}(\operatorname{cond}(\Psi))<\rho$. Finally, let $e=\operatorname{ord}_{\mathfrak{q}}\left(\operatorname{cond}\left(\Psi \Phi^{2}\right)\right)$. Then

$$
\mathcal{S}_{k}^{+}\left(\mathfrak{q}^{\nu} \mathcal{M}, \Psi\right)=\bigoplus_{i=e}^{\nu-1} \mathcal{S}_{k}^{+}\left(\mathfrak{q}^{i} \mathcal{M}, \Psi \Phi^{2}\right)^{\Phi}
$$

Proof. Over the rationals, this is Theorem 3.14 of [3]. Let $\mathbf{f} \in \mathcal{S}_{k}^{+}\left(\mathfrak{q}^{\nu} \mathcal{M}, \Psi\right)$ be a normalized newform. By Theorem 6.10iii, the exact level of $\mathbf{f}_{\Phi}$ divides $\mathfrak{q}^{\nu-1} \mathcal{M}$. By Theorem $5.8, \mathbf{f}_{\Phi}=\mathbf{g}-\mathbf{g}\left|T_{\mathfrak{q}}\right| B_{\mathfrak{q}}$ where $\mathbf{g}$ is a normalized newform in $\mathcal{S}_{k}\left(\mathfrak{q}^{\mu} \mathcal{M}, \Psi \Phi^{2}\right)$. It is easy to se that $e \leq \mu \leq \nu-1$ for if $\mu \geq \nu$ then by Theorem 3.3iii, $\mathbf{g} \mid T_{\mathfrak{q}}=0$ which would imply $\mathbf{f}_{\Phi}=\mathbf{g}$ and hence $\mu<\nu$, a contradiction. Now $\mathbf{f}_{\Phi \bar{\Phi}}=\mathbf{g}_{\bar{\Phi}} \in$ $\mathcal{S}_{k}\left(\mathfrak{q}^{\nu} \mathcal{M}, \Psi\right)$ by Theorem 5.1 and so $\mathbf{g}_{\bar{\Phi}} \sim \mathbf{f}$. Since $\mathbf{f}$ is a newform in $\mathcal{S}_{k}\left(\mathfrak{q}^{\nu} \mathcal{M}, \Psi\right)$, we have $\mathbf{f}=\mathbf{g}_{\bar{\Phi}}$ by Theorem 3.5 (multiplicity one). It follows that

$$
\mathcal{S}_{k}^{+}\left(\mathfrak{q}^{\nu} \mathcal{M}, \Psi\right) \subset \sum_{i=e}^{\nu-1} \mathcal{S}_{k}^{+}\left(\mathfrak{q}^{i} \mathcal{M}, \Psi \Phi^{2}\right)^{\bar{\Phi}}
$$

Conversely, if $\mathbf{g} \in \mathcal{S}_{k}^{+}\left(\mathfrak{q}^{\mu} \mathcal{M}, \Psi \Phi^{2}\right)$ is a normalized newform, then Theorem 7.1 implies that $\mathbf{g}_{\bar{\Phi}}$ is a newform in $\mathcal{S}_{k}\left(\mathfrak{q}^{\nu} \mathcal{M}, \Psi\right)$, thus

$$
\mathcal{S}_{k}^{+}\left(\mathfrak{q}^{\nu} \mathcal{M}, \Psi\right)=\sum_{i=e}^{\nu-1} \mathcal{S}_{k}^{+}\left(\mathfrak{q}^{i} \mathcal{M}, \Psi \Phi^{2}\right)^{\bar{\Phi}}
$$

That the sum on the right hand side is direct follows immediately from multiplicity one theorem (Theorem 3.5).

\section{REFERENCES}

1. A. O. L. Atkin and W. Li, Twists of newforms and pseudo-eigenvalues of W-operators, Invent. Math. 48 (1978), $222-243$.

2. D. Flath, Atkin-Lehner Operators, Math. Ann 246 (1980), 121 - 123.

3. H. Hijikata, A. Pizer, T. Shemanske, Twists of Newforms, J. Number Theory 35 no. 3 (1990), $287-324$.

4. G. Janusz, Algebraic Number Fields, Academic Press, New York, San Francisco, London, 1973.

5. W. Li, Newforms and Functional Equations, Math. Ann 212 (1975), 285 - 315.

6. W. Li (personal communication (1980)).

7. T. Miyake, On automorphic forms on GL(2) and Hecke operators, Ann. of Math 94 (1971), $174-189$.

8. A. Ogg, On the eigenvalues of Hecke operators, Math. Ann 179 (1969), 101 - 108.

9. F. Shahidi, Best Estimates for Fourier Coefficients of Maass Forms, Automorphic Forms and Analytic Number Theory, Centre de Recherches Mathématiques, Montreal, 1990.

10. G. Shimura, Introduction to the Arithmetic Theory of Automorphic Functions, Princeton University Press, Princeton, 1971.

11. G. Shimura, The Special Values of the Zeta Functions Associated with Hilbert Modular Forms, Duke Math. J. 45 (1978), 637-679; Corrections to 'The Special Values of the Zeta Functions Associated with Hilbert Modular Forms', Duke Math. J. 48 (1981), 697.

12. G. Shimura, The arithmetic of certain zeta functions and automorphic forms on orthogonal groups, Annals of Math 111 (1980), 313-375.

13. G. Shimura, On Hilbert Modular Forms of Half-Integral Weight, Duke Math. J. 55 (1987), $765-838$. 
14. L. Walling, On lifting Hecke eigenforms, Trans. AMS (to appear 1991).

15. A. Weil, Basic Number Theory, Springer-Verlag, Berlin, Heidelberg, New York, 1974.

Department of Mathematics, Dartmouth College, Hanover, New Hampshire 03755

E-mail address: Thomas.Shemanske@dartmouth.edu

Department of Mathematics, University of Colorado, Boulder, Colorado 80309 E-mail address: walling@euclid.Colorado.edu 